\title{
THE HODGE-DE RHAM THEORY OF MODULAR GROUPS
}

\author{
RICHARD HAIN
}

Contents

1. Introduction 1

2. Preliminaries 5

Part 1. Completed Path Torsors of Affine Curves 7

3. Relative Completion in the Abstract $\quad 7$

4. A Concrete Approach to Relative Completion 13

5. Relative Completion of Path Torsors 19

6. Zucker's Mixed Hodge Complex 20

7. Relative Completion of Fundamental Groups of Affine Curves 24

8. Completed Path Torsors and Admissible Variations of MHS 37

Part 2. Completed Path Torsors of Modular Curves 38

9. The Variation of Hodge Structure $\mathbb{H} \quad 38$

10. Representation Theory of $\mathrm{SL}_{2} \quad 42$

11. Modular Forms and Eichler-Shimura 43

12. Hodge Theory of the Relative Completion of Modular Groups 45

13. VMHS associated to Modular Forms and their Period Maps 49

14. The Relative Completion of $\pi_{1}\left(\mathcal{M}_{1, \overrightarrow{1}}, x\right) \quad 54$

15. The Monodromy Representation 57

16. The Eisenstein Quotient of a Completed Modular Group 60

17. Modular Symbols and Pollack's Quadratic Relations 62

18. Deligne Cohomology and Extensions of VMHS 68

19. Cup Products and Relations in $\mathfrak{u}^{\text {eis }} \quad 70$

$\begin{array}{ll}\text { References } & 73\end{array}$

\section{Introduction}

The completion $\mathcal{G}_{\Gamma}$ of a finite index subgroup $\Gamma$ of $\mathrm{SL}_{2}(\mathbb{Z})$ with respect to the inclusion $\rho: \Gamma \hookrightarrow \mathrm{SL}_{2}(\mathbb{Q})$ is a proalgebraic group, defined over $\mathbb{Q}$, which is an extension

$$
1 \rightarrow \mathcal{U}_{\Gamma} \rightarrow \mathcal{G}_{\Gamma} \rightarrow \mathrm{SL}_{2} \rightarrow 1
$$

of $\mathrm{SL}_{2}$ by a prounipotent group $\mathcal{U}_{\Gamma}$, and a Zariski dense homomorphism $\Gamma \rightarrow \mathcal{G}_{\Gamma}(\mathbb{Q})$. The main result of [15] implies that, for each choice of a base point of the associated orbi-curve $X_{\Gamma}=\Gamma \rrbracket \mathfrak{h}$, the coordinate ring $\mathcal{O}\left(\mathcal{G}_{\Gamma}\right)$ has a canonical mixed Hodge

Date: July 14, 2015.

Supported in part by grant DMS-1005675 from the National Science Foundation. 
structure (MHS) that is compatible with its product, coproduct and antipode. This MHS induces one on the Lie algebra $\mathfrak{g}_{\Gamma}$ of $\mathcal{G}_{\Gamma} \cdot{ }^{1}$

In this paper we give a detailed exposition of the construction and basic properties of the natural MHS on (the coordinate ring and Lie algebra of) relative completions of modular groups. Part 1 is an exposition of the basic properties of relative completion. It also contains a direct construction of the MHS on the relative completion of the fundamental group (and, more generally, of path torsors) of a smooth affine (orbi) curve with respect to the monodromy representation of a polarized variation of Hodge structure (PVHS). Part 2 is an exploration of the MHS on relative completions of modular groups and their associated path torsors, especially in the case of the full modular group $\mathrm{SL}_{2}(\mathbb{Z})$.

Completions of modular groups are interesting because of their relationship to modular forms and to categories of admissible variations of MHS over modular curves. Because the inclusion $\Gamma \rightarrow \mathrm{SL}_{2}(\mathbb{Q})$ is injective, one might expect the prounipotent radical $\mathcal{U}_{\Gamma}$ of $\mathcal{G}_{\Gamma}$ to be trivial. However, this is not the case. Its Lie algebra $\mathfrak{u}_{\Gamma}$ is a pronilpotent Lie algebra freely topologically generated (though not canonically) by

$$
\prod_{m \geq 0} H^{1}\left(\Gamma, S^{m} H\right)^{*} \otimes S^{m} H
$$

where $H$ denotes the defining representation of $\mathrm{SL}_{2}$ and $S^{m} H$ its $m$ th symmetric power. Because $H^{1}\left(\Gamma, S^{m} H\right)$ is a space of modular forms of $\Gamma$ of weight $m+2$ (Eichler-Shimura), there should be a close relationship between the MHS on $\mathcal{G}_{\Gamma}$ and the geometry and arithmetic of elliptic curves.

To explain the connection with admissible variations of MHS, consider the category $\operatorname{MHS}\left(X_{\Gamma}, \mathbb{H}\right)$ of admissible variations of MHS $\mathbb{V}$ over $X_{\Gamma}$ whose weight graded quotients have the property that the monodromy representation

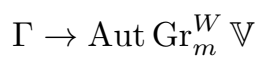

factors through an action of the algebraic group $\mathrm{SL}_{2}$ for all $m$. The monodromy representation

$$
\Gamma \cong \pi_{1}\left(X_{\Gamma}, x\right) \rightarrow \operatorname{Aut} V_{x}
$$

of such a variation $\mathbb{V}$ factors through the canonical homomorphism $\Gamma \rightarrow \mathcal{G}_{\Gamma}(\mathbb{Q})$, so that one has a natural coaction

$$
V_{x} \rightarrow V_{x} \otimes \mathcal{O}\left(\mathcal{G}_{\Gamma}\right) .
$$

In Section 8 we show that there is an equivalence of categories between $\operatorname{MHS}\left(X_{\Gamma}, \mathbb{H}\right)$ and the category of "Hodge representations" of $\mathcal{G}_{\Gamma}$ - that is the category of representations of $\Gamma$ on a MHS $V$ that induce a homomorphism $\mathcal{G}_{\Gamma} \rightarrow$ Aut $V$ for which the coaction (1.2) is a morphism of MHS. The prounipotent radical $\mathcal{U}_{\Gamma}$ of $\mathcal{G}_{\Gamma}$, and hence modular forms via (1.1), control extensions in $\operatorname{MHS}\left(X_{\Gamma}, \mathbb{H}\right)$. This is a special case of a more general result which is proved in [24].

Modular forms give simple extensions in $\operatorname{MHS}\left(X_{\Gamma}, \mathbb{H}\right)$. The fundamental representation $H$ of $\mathrm{SL}_{2}$ corresponds to the polarized variation of Hodge structure $\mathbb{H}$ of weight 1 over $X_{\Gamma}$ whose fiber over the point $x \in X_{\Gamma}$ is the first cohomology group of the corresponding elliptic curve. The classification of admissible variations of MHS

\footnotetext{
${ }^{1}$ There is more structure: if $X_{\Gamma}$ is defined over the number field $K$ and if $x \in X_{\Gamma}$ is a $K$ rational point, then one also has a Galois action on $\mathcal{G} \otimes \mathbb{Q}_{\ell}$. This and the canonical MHS on $\mathcal{G}_{\Gamma}$ should be the Hodge and $\ell$-adic étale realizations of a motivic structure on $\mathcal{G}$ that depends on $x$.
} 
over $X_{\Gamma}$ in the previous paragraph and the computation (1.1) imply that there are extensions of variations

$$
0 \rightarrow H^{1}\left(X_{\Gamma}, S^{m} \mathbb{H}\right)^{*} \otimes S^{m} \mathbb{H} \rightarrow \mathbb{E} \rightarrow \mathbb{Q} \rightarrow 0 .
$$

When $\Gamma$ is a congruence subgroup, this variation splits as the sum of extensions

$$
0 \rightarrow \check{M}_{f} \otimes S^{m} \mathbb{H} \rightarrow \mathbb{E}_{f} \rightarrow \mathbb{Q} \rightarrow 0,
$$

where $f$ is a normalized Hecke eigenform of weight $m+2, M_{f}$ is the corresponding Hodge structure, and $\check{M}_{f}=M_{f}(m+1)$ is its dual. When $f$ is a cusp form, $\check{M}_{f} \otimes S^{m} \mathbb{H}$ has weight -1 . In the case where $\Gamma=\mathrm{SL}_{2}(\mathbb{Z})$, we give an explicit construction of these extensions and the corresponding normal functions in Section 13.4. When $f$ is an Eisenstein series, $\check{M}_{f}=\mathbb{Q}(m+1)$ and the extension is of the form

$$
0 \rightarrow S^{m} \mathbb{H}(m+1) \rightarrow \mathbb{E}_{f} \rightarrow \mathbb{Q} \rightarrow 0 .
$$

These extensions are constructed explicitly in Section 13.3 when $\Gamma=\mathrm{SL}_{2}(\mathbb{Z})$. They correspond to the elliptic polylogarithms of Beilinson and Levin [1].

This work also generalizes and clarifies Manin's work on "iterated Shimura integrals" $[29,30]$. The exact relationship is discussed in Section 13.2. The periods of the MHS on $\mathcal{O}\left(\mathcal{G}_{\Gamma}\right)$ are iterated integrals (of the type defined in [15]) of the logarithmic forms in Zucker's mixed Hodge complex that computes the MHS on the cohomology groups $H^{1}\left(X_{\Gamma}, S^{m} \mathbb{H}\right)$, whose definition is recalled in Section 6. Manin's iterated Shimura integrals are iterated integrals of elements of the subcomplex of holomorphic forms in Zucker's complex. They form a Hopf algebra whose spectrum is a quotient $\mathcal{U}_{A}$ of $\mathcal{U}_{\Gamma}$ by the normal subgroup generated by $F^{0} \mathcal{U}_{\Gamma}$. This quotient is not motivic as it does not support a MHS for which the quotient mapping $\mathcal{U}_{\Gamma} \rightarrow \mathcal{U}_{A}$ is a morphism of MHS. There is a further quotient $\mathcal{U}_{B}$ of $\mathcal{U}_{A}$ that is dual to the Hopf algebra generated by iterated integrals of Eisenstein series. In Section 19 we show that it is not motivic by relating it to the Eisenstein quotient of $\mathcal{U}_{\Gamma}$, described below.

Fix a base point $x \in X_{\Gamma}$, so that $\mathcal{G}_{\Gamma}$ denotes the completion of $\pi_{1}\left(X_{\Gamma}, x\right) \cong \Gamma$ with its natural MHS. The "Eisenstein quotient" $\mathcal{G}_{\Gamma}^{\text {eis }}$ of $\mathcal{G}_{\Gamma}$, defined in Section 16, is the maximal quotient of $\mathcal{G}_{\Gamma}$ whose Lie algebra $\mathfrak{g}_{\Gamma}^{\text {eis }}$ has a MHS whose weight graded quotients are sums of Tate twists of the natural Hodge structure on $S^{n} H_{x}$. Its isomorphism type does not depend on the base point $x$. As $x$ varies in $X_{\Gamma}$, the coordinate rings of the Eisenstein quotients form an admissible VMHS over $X_{\Gamma}$.

Denote the Lie algebra of $\mathcal{U}_{B}$ by $\mathfrak{u}_{B}$ and of the prounipotent radical $\mathcal{U}_{\Gamma}^{\text {eis }}$ of $\mathcal{G}_{\Gamma}^{\text {eis }}$ by $\mathfrak{u}_{\Gamma}^{\text {eis }}$. Since the Hodge structure $\check{M}_{f} \otimes S^{n} H_{x}$ associated to an eigencuspform $f$ is not of this type, such Hodge structures will lie in the kernel of

$$
H_{1}\left(\mathfrak{u}_{\Gamma}\right) \rightarrow H_{1}\left(\mathfrak{u}_{\Gamma}^{\text {eis }}\right),
$$

which implies that $H_{1}\left(\mathfrak{u}_{\Gamma}^{\text {eis }}\right)$ is generated by one copy of $S^{m} H_{x}(m+1)$ for each normalized Eisenstein series of weight $m+2$. In particular, when $\Gamma=\mathrm{SL}_{2}(\mathbb{Z})$,

$$
H_{1}\left(\mathfrak{u}_{\Gamma}^{\mathrm{eis}}\right) \cong \prod_{n \geq 1} S^{2 n} H_{x}(2 n+1)
$$

There is a natural projection $\mathcal{U}_{B} \rightarrow \mathcal{U}_{\Gamma}^{\text {eis }}$ from Manin's quotient of $\mathcal{U}_{\Gamma}$ to $\mathcal{U}_{\Gamma}^{\text {eis }}$ that induces an isomorphism

$$
H_{1}\left(\mathfrak{u}_{B}\right) \cong H_{1}\left(\mathfrak{u}_{\Gamma}^{\mathrm{eis}}\right) .
$$


But, as we show in Section 19, the cuspidal generators $\check{M}_{f} \otimes S^{2 n} H_{x}$ of $\mathfrak{u}_{\Gamma}$ become non-trivial relations in $\mathfrak{u}_{\Gamma}^{\text {eis }}$. Such relations were suggested by computations in the $\ell$-adic étale version with Makoto Matsumoto (cf. [23]). Evidence for them was provided by Aaron Pollack's undergraduate thesis [33] in which he found quadratic relations between the generators of the image of the representation $\operatorname{Gr}_{\bullet}^{W} \mathfrak{u}_{\Gamma}^{\text {eis }} \rightarrow \operatorname{Der} \mathbb{L}(H)$ induced by the natural action of $\mathcal{U}_{\Gamma}^{\text {eis }}$ on the unipotent fundamental group of a once punctured elliptic curve, which we construct in Section $15 .^{2}$ The arguments in Section 19 and the computations of Brown [3] and Terasoma [43] (Thm. 19.3) imply that Pollack's quadratic relations also hold in $\mathfrak{u}_{\Gamma}^{\text {eis }}$. Since $\mathfrak{u}_{B}$ is free and since $\mathfrak{u}^{\text {eis }}$ is not, Manin's quotient $\mathfrak{u}_{B}$ does not support a natural MHS.

The starting point of much of this work is the theory of "universal mixed elliptic motives" [23] developed with Makoto Matsumoto. The origin of that project was a computation in 2007 of the $\ell$-adic weighted completion of $\pi_{1}\left(\mathcal{M}_{1,1 / \mathbb{Z}[1 / \ell]}\right)$ in which we observed that cuspidal generators of the relative completion of the geometric fundamental group of $\mathcal{M}_{1,1 / \mathbb{Z}[1 / \ell]}$ appeared to become relations in the weighted completion of its arithmetic fundamental group. Pollack's thesis [33] added evidence that these cuspidal generators had indeed become relations in the weighted completion.

Finally, we mention related work by Levin and Racinet [28], Brown and Levin [4], and Calaque, Enriquez and Etingof [6], and subsequent work of Enriquez.

Although the paper contains many new results, it is expository. The intended audience is somebody who is familiar with modern Hodge theory. Several standard topics, such as a discussion of modular symbols, are included to fix notation and point of view, and also to make the paper more accessible. The reader is assumed to be familiar with the basics of mixed Hodge structures, their construction and their variations, including the basics of computing limit mixed Hodge structures.

Acknowledgments: It is a pleasure to acknowledge the mathematicians with whom I have had fruitful discussions, which helped shape my view of the subject of these notes. In particular, I would like to thank my long term collaborator, Makoto Matsumoto, as well as Aaron Pollack and Francis Brown. I am indebted to Francis Brown and Tomohide Terasoma, each of whom communicated their computation of the cup product, Theorem 19.3. I am also grateful to Francis Brown for his interest in the project and for his numerous constructive comments and corrections.

\subsection{Notation and Conventions.}

1.1.1. Path multiplication and iterated integrals. In this paper we use the topologist's convention (which is the opposite of the algebraist's convention) for path multiplication. Two paths $\alpha, \beta:[0,1] \rightarrow X$ in a topological space $X$ are composable when $\alpha(1)=\beta(0)$. The product $\alpha * \beta$ of two composable paths first traverses $\alpha$ and then $\beta$.

Denote the complex of smooth $\mathbb{C}$-valued forms on a smooth manifold $M$ by $E^{\bullet}(M)$. Iterated integrals are defined using Chen's original definition: if $\omega_{1}, \ldots, \omega_{r} \in$

\footnotetext{
${ }^{2} \mathrm{He}$ also found, for each cusp form, relations of all degrees $\geq 3$ that hold in a certain quotient of the image of this representation.
} 
$E^{1}(M) \otimes A$ are 1-forms on a manifold $M$ that take values in an associative $\mathbb{C}$-algebra $A$ and $\alpha:[0,1] \rightarrow M$ is a piecewise smooth path, then

$$
\int_{\alpha} \omega_{1} \omega_{2} \ldots \omega_{r}=\int_{\Delta^{r}} f_{1}\left(t_{1}\right) \ldots f_{r}\left(t_{r}\right) d t_{1} d t_{2} \ldots d t_{r} .
$$

where $f_{j}(t) d t=\alpha^{*} \omega_{j}$ and $\Delta^{r}$ is the "time ordered" $r$-simplex

$$
\Delta^{r}=\left\{\left(t_{1}, \ldots, t_{r}\right) \in \mathbb{R}^{n}: 0 \leq t_{1} \leq t_{2} \leq \cdots \leq t_{r} \leq 1\right\} .
$$

An exposition of the basic properties of iterated integrals can be found in [13, 17].

1.1.2. Filtrations. The lower central series (LCS) $L^{\bullet} G$ of a group $G$ is defined by

$$
G=L^{1} G \supseteq L^{2} G \supseteq L^{3} G \supseteq \cdots
$$

where $L^{m+1} G=\left[G, L^{m} G\right]$. Its associated graded $\operatorname{Gr}_{\mathrm{LCS}}^{\bullet} G$ is a graded Lie algebra over $\mathbb{Z}$ whose $m$ th graded quotient is $\operatorname{Gr}_{\mathrm{LCS}}^{m} G:=L^{m} G / L^{m+1} G$.

The lower central series $L \cdot \mathfrak{g}$ of a Lie algebra $\mathfrak{g}$ is defined similarly. A Lie algebra $\mathfrak{g}$ is nilpotent if $L^{N} \mathfrak{g}=0$ for some $N \geq 0$.

1.1.3. Hodge theory. All mixed Hodge structures will be $\mathbb{Q}$ mixed Hodge structures unless otherwise stated. The category of $\mathbb{Q}$-mixed Hodge structures will be denoted by MHS. The category of $\mathbb{R}$-mixed Hodge structures will be denoted by $M H S_{\mathbb{R}}$.

Often we will abbreviate mixed Hodge structure by MHS, variation of MHS by VMHS, mixed Hodge complex by MHC, cohomological MHC by CMHC. The category of admissible VMHS over a smooth variety $X$ will be denoted by $\operatorname{MHS}(X)$.

\section{Preliminaries}

2.1. Proalgebraic groups. In this paper, the term algebraic group will refer to a linear algebraic group. Suppose that $F$ is a field of characteristic zero. A proalgebraic group $G$ over $F$ is an inverse limit of algebraic $F$-groups $G_{\alpha}$. The coordinate ring $\mathcal{O}(G)$ of $G$ is the direct limit of the coordinate rings of the $G_{\alpha}$. The Lie algebra $\mathfrak{g}$ of $G$ is the inverse limit of the Lie algebras $\mathfrak{g}_{\alpha}$ of the $G_{\alpha}$. It is a Hausdorff topological Lie algebra. The neighbourhoods of 0 are the kernels of the canonical projections $\mathfrak{g} \rightarrow \mathfrak{g}_{\alpha}$.

The continuous cohomology of $\mathfrak{g}=\lim _{\mathfrak{g}} \mathfrak{g}_{\alpha}$ is defined by

$$
H^{\bullet}(\mathfrak{g}):=\underset{\alpha}{\lim } H^{\bullet}\left(\mathfrak{g}_{\alpha}\right) .
$$

Its homology is the full dual:

$$
H_{\bullet}(\mathfrak{g}):=\operatorname{Hom}_{F}\left(H^{\bullet}(\mathfrak{g}), F\right) \cong \lim _{\longleftarrow} H_{\bullet}\left(\mathfrak{g}_{\alpha}\right)
$$

Each homology group is a Hausdorff topological vector space; the neighbourhoods of 0 are the kernels of the natural maps $H_{\bullet}(\mathfrak{g}) \rightarrow H_{\bullet}\left(\mathfrak{g}_{\alpha}\right)$.

Continuous cohomology can be computed using continuous Chevalley-Eilenberg cochains:

$$
\mathcal{C}^{\bullet}(\mathfrak{g})=\operatorname{Hom}_{F}^{\text {cts }}\left(\Lambda^{\bullet} \mathfrak{g}, F\right):=\underset{\alpha}{\lim } \operatorname{Hom}_{F}\left(\Lambda^{\bullet} \mathfrak{g}_{\alpha}, F\right)
$$

with the usual differential.

If, instead, $\mathfrak{g}=\bigoplus_{m} \mathfrak{g}_{m}$ is a graded Lie algebra, then the homology and cohomology of $\mathfrak{g}$ are also graded. This follows from the fact that the grading of $\mathfrak{g}$ induces a grading of the Chevalley-Eilenberg chains and cochains of $\mathfrak{g}$. 
2.2. Prounipotent groups and pronilpotent Lie algebras. A prounipotent $F$-group is a proalgebraic group that is an inverse limit of unipotent $F$-groups.

A pronilpotent Lie algebra over a $F$ is an inverse limit of finite dimensional nilpotent Lie algebras. The Lie algebra of a prounipotent group is a pronilpotent Lie algebra. The functor that takes a prounipotent group to its Lie algebra is an equivalence of categories between the category of unipotent $F$-groups and the category of pronilpotent Lie algebras over $F$.

The following useful result is an analogue for pronilpotent Lie algebras of a classical result of Stallings [40]. A proof can be found in [18, §3].

Proposition 2.1. For a homomorphism $\varphi: \mathfrak{n}_{1} \rightarrow \mathfrak{n}_{2}$ of pronilpotent Lie algebras, the following are equivalent:

(i) $\varphi$ is an isomorphism,

(ii) $\varphi^{*}: H^{\bullet}\left(\mathfrak{n}_{2}\right) \rightarrow H^{\bullet}\left(\mathfrak{n}_{1}\right)$ is an isomorphism,

(iii) $\varphi^{*}: H^{j}\left(\mathfrak{n}_{2}\right) \rightarrow H^{j}\left(\mathfrak{n}_{1}\right)$ is an isomorphism when $j=1$ and injective when $j=2$.

Another useful fact that we shall need is the following exact sequence, which is essentially due to Sullivan [42].

Proposition 2.2. If $\mathfrak{n}$ is a pronilpotent Lie algebra over $F$, then the sequence

$$
0 \longrightarrow\left(\operatorname{Gr}_{\mathrm{LCS}}^{2} \mathfrak{n}\right)^{*} \stackrel{[,]^{*}}{\longrightarrow} \Lambda^{2} H^{1}(\mathfrak{n}) \stackrel{c u p}{\longrightarrow} H^{2}(\mathfrak{n})
$$

is exact, where ()$^{*}=\operatorname{Hom}^{\mathrm{cts}}(, F)$ and $[,]^{*}$ denotes the continuous dual of the bracket $\Lambda^{2} H_{1}(\mathfrak{n}) \rightarrow \mathrm{Gr}_{\mathrm{LCS}}^{2} \mathfrak{n}$.

Remark 2.3. When $H_{1}(\mathfrak{n})$ is finite dimensional, one can dualize to obtain the exact sequence

$$
H_{2}(\mathfrak{n}) \stackrel{\text { cup }^{*}}{\longrightarrow} \Lambda^{2} H_{1}(\mathfrak{n}) \stackrel{[,]}{\longrightarrow} \operatorname{Gr}_{\mathrm{LCS}}^{2} \mathfrak{n} \longrightarrow 0 .
$$

However, when $H_{1}(\mathfrak{n})$ is infinite dimensional, this sequence is not exact. Instead, one needs to replace $\Lambda^{2} H_{1}(\mathfrak{n})$ by the alternating part of the completed tensor product $H_{1}(\mathfrak{n}) \hat{\otimes} H_{1}(\mathfrak{n})$.

2.3. Free Lie algebras. Suppose that $F$ is a field of characteristic 0 and that $V$ is a vector space over $F$. Here we are not assuming $V$ to be finite dimensional. The free Lie algebra generated by $V$ will be denoted by $\mathbb{L}(V)$. It is characterized by the property that a linear map $V \rightarrow \mathfrak{g}$ into a Lie algebra over $F$ induces a unique Lie algebra homomorphism $\mathbb{L}(V) \rightarrow \mathfrak{g}$. The Poincaré-Birkhoff-Witt Theorem implies [37] that $\mathbb{L}(V)$ is the Lie subalgebra of the tensor algebra $T(V)$ (with bracket $[A, B]=A B-B A)$ generated by $V$ and that the inclusion $\mathbb{L}(V) \rightarrow T(V)$ induces an isomorphism $U \mathbb{L}(V) \rightarrow T(V)$ from the enveloping algebra of $\mathbb{L}(V)$ to $T(V)$. The cohomology of $\mathbb{L}(V)$ with trivial coefficients vanishes in degrees $>1$.

If $\mathfrak{f}$ is a Lie algebra, then any splitting of the projection $\mathfrak{f} \rightarrow H_{1}(\mathfrak{f})$ induces a homomorphism $\mathbb{L}\left(H_{1}(\mathfrak{f})\right) \rightarrow \mathfrak{f}$. If $\mathfrak{f}$ is free, then this homomorphism is an isomorphism [37]. It induces a canonical isomorphism

$$
\operatorname{Gr}_{\mathrm{LCS}}^{\bullet} \mathfrak{f} \cong \mathbb{L}\left(H_{1}(\mathfrak{f})\right)
$$

of the graded Lie algebra associated to the lower central series (LCS) of $\mathfrak{f}$ with the free Lie algebra generated by its first graded quotient $H_{1}(\mathfrak{f})=\mathfrak{f} / L^{2} \mathfrak{f}$. 
The free completed Lie algebra $\mathbb{L}(V)^{\wedge}$ generated by $V$ is defined to be

$$
\mathbb{L}(V)^{\wedge}=\lim _{\longleftarrow} \mathfrak{n},
$$

where $\mathfrak{n}$ ranges over all finite dimensional nilpotent quotients of $\mathbb{L}(V)$. It is viewed as a topological Lie algebra. It is useful to note that there is a canonical isomorphism

$$
\mathbb{L}(V)^{\wedge}=\lim _{W, n} \mathbb{L}(W) / L^{n} \mathbb{L}(W)
$$

of topological Lie algebras, where $W$ ranges over all finite dimensional quotients of $V$ and $n$ over all positive integers.

We can regard $V$ as a topological vector space: the neighbourhoods of 0 are the subspaces of $V$ of finite codimension. Every continuous linear mapping $V \rightarrow \mathfrak{u}$ from $V$ into a pronilpotent Lie algebra induces a unique continuous homomorphism $\mathbb{L}(V)^{\wedge} \rightarrow \mathfrak{u}$. The continuous cohomology of $\mathbb{L}(V)^{\wedge}$ vanishes in degrees $\geq 2$.

If $\mathfrak{n}$ is a pronilpotent Lie algebra, then any continuous section of the quotient mapping $\mathfrak{n} \rightarrow H_{1}(\mathfrak{n})$ induces a continuous surjective homomorphism $\mathbb{L}\left(H_{1}(\mathfrak{n})\right)^{\wedge} \rightarrow$ $\mathfrak{n}$. Applying Proposition 2.1 to this homomorphism, we obtain:

Proposition 2.5. A pronilpotent Lie algebra is free if and only if $H^{2}(\mathfrak{n})=0$.

\section{Part 1. Completed Path Torsors of Affine Curves}

\section{Relative Completion in the Abstract}

Suppose that $\Gamma$ is a discrete group and that $R$ is a reductive algebraic group over a field $F$ of characteristic zero. The completion of $\Gamma$ relative to a Zariski dense representation $\rho: \Gamma \rightarrow R(F)$ is a proalgebraic $F$-group $\mathcal{G}$ which is an extension

$$
1 \rightarrow \mathcal{U} \rightarrow \mathcal{G} \rightarrow R \rightarrow 1
$$

of $R$ by a prounipotent group $\mathcal{U}$, and a homomorphism $\hat{\rho}: \Gamma \rightarrow \mathcal{G}(F)$ such that the composite

$$
\Gamma \stackrel{\hat{\rho}}{\longrightarrow} \mathcal{G}(F) \longrightarrow R(F)
$$

is $\rho$. It is universal for such groups: if $G$ is a proalgebraic $F$ group that is an extension of $R$ by a prounipotent group, and if $\phi: \Gamma \rightarrow G(F)$ is a homomorphism whose composition with $G \rightarrow R$ is $\rho$, then there is a homomorphism $\hat{\phi}: \mathcal{G} \rightarrow G$ of proalgebraic $F$-groups such that the diagram

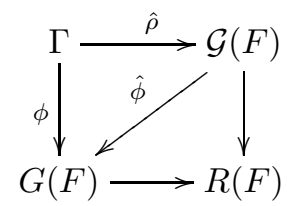

commutes.

When $R$ is trivial, $\rho$ is trivial and $\mathcal{G}=\mathcal{U}$ is the unipotent completion of $\Gamma$ over $F$.

Relative completion can be defined as follows: Let $\mathcal{L}(\Gamma, R)$ denote the category of finite dimensional $F$-linear representations $V$ of $\Gamma$ that admit a filtration

$$
0=V_{0} \subset V_{1} \subset \cdots \subset V_{N}=V
$$

by $\Gamma$-submodules with the property that each graded quotient $V_{j} / V_{j-1}$ is an $R$ module and the action of $\Gamma$ on it factors through $\rho$. It is a neutral tannakian 
category. The completion of $\Gamma$ relative to $\rho$ is the fundamental group of this category with respect to the fiber functor that takes a representation to its underlying vector space.

We will generally be sloppy and not distinguish between a proalgebraic group $G$ and its group $G(F)$ of $F$-rational points. For example, in the context of relative completion, $\rho$ will be a homomorphism $\Gamma \rightarrow R$.

3.1. Levi splittings. The following generalization of Levi's Theorem implies that the relative completion $\mathcal{G}$ of a finitely generated group $\Gamma$ can be expressed (noncanonically) as a semi-direct product $\mathcal{G} \cong R \ltimes \mathcal{U}$. The Lie algebra $\mathfrak{u}$ of $\mathcal{U}$ is then a pronilpotent Lie algebra in the category of $R$-modules. The isomorphism type of $\mathcal{G}$ is determined by $\mathfrak{u}$ with its $R$-action.

Suppose that $F$ is a field of characteristic 0 and that $R$ is a reductive $F$-group. Call an extension

$$
1 \rightarrow \mathcal{U} \rightarrow \mathcal{G} \rightarrow R \rightarrow 1
$$

of $R$ by a prounipotent group in the category of affine $F$-groups quasi-finite if for all finite dimensional $R$-modules $V, \operatorname{Hom}_{R}\left(V, H_{1}(\mathcal{U})\right)$ is finite dimensional. The results in the following section imply that the completion of a finitely generated group $\Gamma$ relative to a homomorphism $\rho: \Gamma \rightarrow R(F)$ is a quasi finite extension of $R$.

Proposition 3.1. Every quasi-finite extension of $R$ by a prounipotent group $\mathcal{U}$ is split. Moreover, any two splittings are conjugate by an element of $\mathcal{U}(F)$.

Sketch of Proof. The classical case where $\mathcal{U}$ is an abelian unipotent group (i.e., a finite dimensional vector space) was proved by Mostow in [32]. (See also, [2, Prop. 5.1].)

First consider the case where $\mathcal{U}$ is an abelian proalgebraic group. The quasifiniteness assumption implies that there are (finite dimensional) abelian unipotent groups $\mathcal{U}_{\alpha}$ with $R$-action and an $R$-equivariant isomorphism

$$
\mathcal{U} \cong \prod_{\alpha} \mathcal{U}_{\alpha} .
$$

The extension of $\mathcal{G}$ by $\mathcal{U}$ can be pushed out along the projection $\mathcal{U} \rightarrow \mathcal{U}_{\alpha}$ to obtain extensions

$$
1 \rightarrow \mathcal{U}_{\alpha} \rightarrow \mathcal{G}_{\alpha} \rightarrow R \rightarrow 1
$$

The classical case, stated above, implies that each of these has a splitting $s_{\alpha}$ and that this splitting is unique up to conjugation by an element of $\mathcal{U}_{\alpha}$. These sections assemble to give a section $s=\left(s_{\alpha}\right)$ of $\mathcal{G} \rightarrow R$ that is defined over $F$. Every section of $\mathcal{G} \rightarrow R$ is of this form. Any two are conjugate by an element of $\mathcal{U}(F)$.

To prove the general case, consider the extensions

$$
1 \rightarrow \mathcal{U}_{n} \rightarrow \mathcal{G}_{n} \rightarrow R \rightarrow 1
$$

where $\mathcal{G}_{n}=\mathcal{G} / L^{n+1} \mathcal{U}, \mathcal{U}_{n}=\mathcal{U} / L^{n+1} \mathcal{U}$, and where $L^{n} \mathcal{U}$ denotes the $n$th term of the LCS of $\mathcal{U}$. The result is proved by constructing a compatible sequence of sections of these extensions. We have already established the $n=1$ case. Suppose that $n>1$ and that we have constructed a splitting of $s_{n-1}$ of $\mathcal{G}_{n-1} \rightarrow R$ and shown that any two such splittings are conjugate by an element of $\mathcal{U}_{n-1}$.

Pulling back the extension

$$
1 \rightarrow \mathrm{Gr}_{\mathrm{LCS}}^{n} \mathcal{U} \rightarrow \mathcal{G}_{n} \rightarrow \mathcal{G}_{n-1} \rightarrow 1
$$


along $s_{n-1}$ gives an extension

$$
1 \rightarrow \mathrm{Gr}_{\mathrm{LCS}}^{n} \mathcal{U} \rightarrow G \rightarrow R \rightarrow 1
$$

The quasi-finite assumption implies that the $R$-module $\operatorname{Gr}_{\mathrm{LCS}}^{n} \mathcal{U}$ is a product of finite dimensional $R$-modules. The $n=1$ case implies that this extension is split and that any two splittings are conjugate by an element of $\operatorname{Gr}_{\mathrm{LCS}}^{n} \mathcal{U}$. If $s$ is a section of $G \rightarrow R$, then the composition of $s$ with the inclusion $G \hookrightarrow \mathcal{G}_{n}$ is a section $s_{n}$ of (3.2) that is compatible with $s_{n-1}$ :

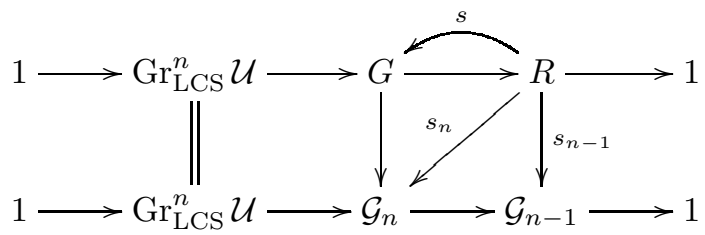

The uniqueness of $s$ implies that any two such lifts of $s_{n-1}$ are conjugate by an element of $\operatorname{Gr}_{\mathrm{LCS}}^{n} \mathcal{U}(F)$. This and the fact that $s_{n-1}$ is unique up to conjugation by an element of $\mathcal{U}(F)$ implies that $s_{n}$ is as well.

3.2. Cohomology. We continue with the notation above, where $\mathcal{G}$ is the relative completion of $\Gamma$. When $R$ is reductive, the structure of $\mathfrak{g}$ and $\mathfrak{u}$ are closely related to the cohomology of $\Gamma$ with coefficients in rational representations of $R$. We will assume also that $H^{j}(\Gamma, V)$ is finite dimensional when $j \leq 2$ for all rational representations $V$ of $R$. This condition is satisfied when $\Gamma$ is finitely presented and thus by fundamental groups of all complex algebraic varieties.

For each rational representation $V$ of $R$ there are natural isomorphisms

$$
\operatorname{Hom}_{R}^{\text {cts }}\left(H_{\bullet}(\mathfrak{u}), V\right) \cong\left[H^{\bullet}(\mathfrak{u}) \otimes V\right]^{R} \cong H^{\bullet}(\mathcal{G}, V) .
$$

The homomorphism $\Gamma \rightarrow \mathcal{G}(F)$ induces a homomorphism

$$
H^{\bullet}(\mathfrak{u}, V)^{R} \cong H^{\bullet}(\mathcal{G}, V) \rightarrow H^{\bullet}(\Gamma, V)
$$

It is an isomorphism in degrees $\leq 1$ and an injection in degree 2 .

Denote the set of isomorphism classes of finite dimensional irreducible representations of $R$ by $\check{R}$. Fix an $R$-module $V_{\lambda}$ in each isomorphism class $\lambda \in \check{R}$. If each irreducible representation of $R$ is absolutely irreducible ${ }^{3}$ and if $H^{j}(\Gamma, V)$ is finite dimensional for all rational representations $V$ of $R$ when $j=1$, 2, then (3.3) implies that there is an isomorphism

$$
\prod_{\lambda \in \check{R}}\left[H^{1}\left(\Gamma, V_{\lambda}\right)\right]^{*} \otimes_{F} V_{\lambda} \cong H_{1}(\mathfrak{u})
$$

of topological modules, and that there is a continuous $R$-invariant surjection

$$
\prod_{\lambda \in \check{R}}\left[H^{2}\left(\Gamma, V_{\lambda}\right)\right]^{*} \otimes_{F} V_{\lambda} \rightarrow H_{2}(\mathfrak{u}) .
$$

In both cases, the LHS has the product topology.

\footnotetext{
${ }^{3}$ This is the case when $R=\mathrm{Sp}_{g}$ over any field of characteristic zero.
} 
3.3. Base change. When discussing the mixed Hodge structure on a relative completion of the fundamental group of a complex algebraic manifold $X$, we need to be able to compare the completion of $\pi_{1}(X, x)$ over $\mathbb{R}($ or $\mathbb{Q})$ with its completion over $\mathbb{C}$. For this reason we need to discuss the behaviour of relative completion under base change.

The cohomological properties of relative completion stated above imply that it behaves well under base change. To explain this, suppose that $K$ is an extension field of $F$. Then $\rho_{K}: \Gamma \rightarrow R(K)$ is Zariski dense in $R \times_{F} K$, so one has the completion $\mathcal{G}_{K}$ of $\Gamma$ relative to $\rho_{K}$. It is an extension of $R \times_{F} K$ by a prounipotent group. The universal mapping property of $\mathcal{G}_{K}$ implies that the homomorphism $\Gamma \rightarrow \mathcal{G}(K)$ induces a homomorphism $\mathcal{G}_{K} \rightarrow \mathcal{G} \times{ }_{F} K$ of proalgebraic $K$-groups. The fact that (3.3) is an isomorphism in degree 1 and injective in degree 2 implies that this homomorphism is an isomorphism.

3.4. Examples. Here the coefficient field $F$ will be $\mathbb{Q}$. But because of base change, the discussion is equally valid when $F$ is any field of characteristic 0 .

3.4.1. Free groups. Suppose that $\Gamma$ is a finitely generated free group and that $\rho$ : $\Gamma \rightarrow R(F)$ is a Zariski dense reductive representation. Denote the completion of $\Gamma$ with respect to $\rho$ by $\mathcal{G}$ and its unipotent radical by $\mathcal{U}$. Denote their Lie algebras by $\mathfrak{g}$ and $\mathfrak{u}$. Since $H^{j}(\Gamma, V)$ vanishes for all $R$-modules $V$ for all $j \geq 2, \mathfrak{u}$ is free. Consequently, the homomorphism (3.3) is an isomorphism in all degrees.

3.4.2. Modular groups. Suppose that $\Gamma$ is a modular group - that is, a finite index subgroup of $\mathrm{SL}_{2}(\mathbb{Z})$. Let $R=\mathrm{SL}_{2}$ and $\rho: \Gamma \rightarrow \mathrm{SL}_{2}(\mathbb{Q})$ be the inclusion. This has Zariski dense image. Denote the completion of $\Gamma$ with respect to $\rho$ by $\mathcal{G}$ and its unipotent radical by $\mathcal{U}$.

Every torsion free subgroup $\Gamma^{\prime}$ of $\mathrm{SL}_{2}(\mathbb{Z})$ is the fundamental group of the quotient $\Gamma^{\prime} \backslash \mathfrak{h}$ of the upper half plane by $\Gamma^{\prime}$. Since this is a non-compact Riemann surface, $\Gamma^{\prime}$ is free. Since $\mathrm{SL}_{2}(\mathbb{Z})$ has finite index torsion free subgroups (e.g., the matrices congruent to the identity $\bmod m$ for any $m \geq 3$ ), every modular group is virtually free. This implies that $H^{j}(\Gamma, V)=0$ whenever $j \geq 2$ and $V$ is a rational vector space. The results of Section 3.2 imply that the Lie algebra $\mathfrak{u}$ of $\mathcal{U}$ is a free pronilpotent Lie algebra. As in the case of a free group, this implies that the homomorphism (3.3) is an isomorphism in all degrees.

The set $\check{R}$ of isomorphism classes of irreducible $R$-modules is $\mathbb{N}$. The natural number $n$ corresponds to the $n$th symmetric power $S^{n} H$ of the defining representation $\mathrm{H}$ of $\mathrm{SL}_{2}$. The results of Section 3.2 imply that there is a non-canonical isomorphism

$$
\mathfrak{u} \cong \mathbb{L}\left(\bigoplus_{n \geq 0} H^{1}\left(\Gamma, S^{n} H\right)^{*} \otimes S^{n} H\right)^{\wedge}
$$

of pronilpotent Lie algebras in the category of $\mathrm{SL}_{2}$ modules. (Cf. Remarks 3.9 and 7.2 in [16].) So we have a complete description of $\mathcal{G}$ as a proalgebraic group:

$$
\mathcal{G} \cong \mathrm{SL}_{2} \ltimes \exp \mathfrak{u} .
$$

In Section 4 we give a method for constructing a homomorphism $\Gamma \rightarrow \mathrm{SL}_{2}(\mathbb{C}) \ltimes \exp \mathfrak{u}$ that induces an isomorphism $\mathcal{G} \rightarrow \mathrm{SL}_{2}(\mathbb{C}) \ltimes \exp \mathfrak{u}$. 
3.4.3. Unipotent completion of fundamental groups of punctured elliptic curves. Here $E$ is a smooth elliptic curve over $\mathbb{C}$ and $\Gamma=\pi_{1}\left(E^{\prime}, x\right)$ where $E^{\prime}=E-\{0\}$ and $x \in E^{\prime}$. In this case we take $R$ to be trivial. The corresponding completion of $\Gamma$ is the unipotent completion of $\pi_{1}\left(E^{\prime}, x\right)$. Since $H^{2}\left(E^{\prime}\right)=0$, the results of Section 3.2 imply that the Lie algebra $\mathfrak{p}$ of the unipotent completion of $\pi_{1}\left(E^{\prime}, x\right)$ is (non-canonically isomorphic to) the completion of the free Lie algebra generated by $H_{1}(E, \mathbb{Q})$ :

$$
\mathfrak{p} \cong \mathbb{L}\left(H_{1}(E)\right)^{\wedge}
$$

This induces a canonical isomorphism $\operatorname{Gr}_{\mathrm{LCS}}^{\bullet} \mathfrak{p} \cong \mathbb{L}\left(H_{1}(E)\right)$ of the associated graded Lie algebra of the lower central series (LCS) of $\mathfrak{p}$ with the free Lie algebra generated by $H_{1}(E)$.

3.5. Naturality and Right exactness. The following naturality property is easily proved using either the universal mapping property of relative completion or its tannakian description.

Proposition 3.5. Suppose that $\Gamma$ and $\Gamma^{\prime}$ are discrete groups and that $R$ and $R^{\prime}$ are reductive F-groups. If one has a commutative diagram

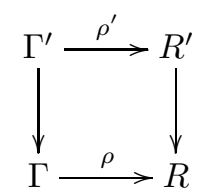

in which $\rho$ and $\rho^{\prime}$ are Zariski dense, then one has a commutative diagram

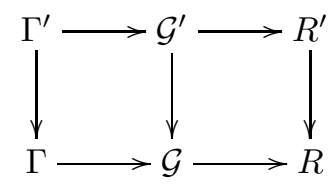

where $\mathcal{G}$ and $\mathcal{G}^{\prime}$ denote the completions of $\Gamma$ and $\Gamma^{\prime}$ with respect to $\rho$ and $\rho^{\prime}$.

Relative completion is not, in general, an exact functor. However, it is right exact. The following is a special case of this right exactness. It can be proved using the universal mapping property of relative completion. (A similar argument can be found in $[22, \S 4.5]$.)

Proposition 3.6. Suppose that $\Gamma, \Gamma^{\prime}$ and $\Gamma^{\prime \prime}$ are discrete groups and that $R, R^{\prime}$ and $R^{\prime \prime}$ are reductive F-groups. Suppose that one has a diagram

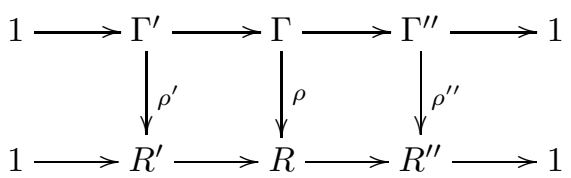

with exact rows in which $\rho, \rho^{\prime}$ and $\rho^{\prime \prime}$ are Zariski dense, then the corresponding diagram

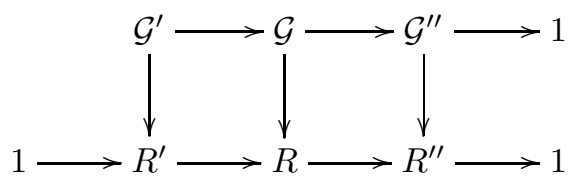

of relative completions has right exact top row. 
Example 3.7. The moduli space of $n \geq 1$ pointed genus 1 curves will be denoted by $\mathcal{M}_{1, n}$. It will be regarded as an orbifold. It is isomorphic to (and will be regarded as) the moduli space of elliptic curves $(E, 0)$ with $n-1$ distinct labelled points $\left(x_{1}, \ldots, x_{n-1}\right)$. The point of $\mathcal{M}_{1, n}$ that corresponds to $\left(E, 0, x_{1}, \ldots, x_{n-1}\right)$ will be denoted by $\left[E, x_{1}, \ldots, x_{n-1}\right]$.

The fiber of the projection $\mathcal{M}_{1,2} \rightarrow \mathcal{M}_{1,1}$ that takes $[E, x]$ to $[E]$ is $E^{\prime}:=$ $E-\{0\}$. Fix a base point $x_{o}=[E, x]$ of $\mathcal{M}_{1,2}$ and $t_{o}=[E] \in \mathcal{M}_{1,1}$. The (orbifold) fundamental group of $\mathcal{M}_{1,2}$ is an extension

$$
1 \rightarrow \pi_{1}\left(E^{\prime}, x\right) \rightarrow \pi_{1}\left(\mathcal{M}_{1,2}, x_{o}\right) \rightarrow \pi_{1}\left(\mathcal{M}_{1,1}, t_{o}\right) \rightarrow 1 .
$$

Denote the completion of $\pi_{1}\left(\mathcal{M}_{1,2}, x_{o}\right)$ with respect to the natural homomorphism to $\mathrm{SL}\left(H_{1}(E)\right) \cong \mathrm{SL}_{2}(\mathbb{Q})$ by $\widetilde{\mathcal{G}}$. Functoriality and right exactness of relative completion implies that we have an exact sequence

$$
\pi_{1}\left(E^{\prime}, x\right)^{\text {un }} \rightarrow \widetilde{\mathcal{G}} \rightarrow \mathcal{G} \rightarrow 1 .
$$

In this case, we can prove exactness on the left as well.

This is proved using the conjugation action of $\pi_{1}\left(\mathcal{M}_{1,2}, x_{o}\right)$ on $\pi_{1}\left(E^{\prime}, x\right)$, which induces an action of $\pi_{1}\left(\mathcal{M}_{1,2}, x_{o}\right)$ on the Lie algebra $\mathfrak{p}$ of $\pi_{1}\left(E^{\prime}, x\right)^{\mathrm{un}}$. This action preserves the lower central series filtration of $\mathfrak{p}$ and therefore induces an action on $\operatorname{Gr}_{\mathrm{LCS}}^{\bullet} \mathfrak{p} \cong \mathbb{L}\left(H_{1}(E)\right)$. This action is determined by its action on $H_{1}(E)$, and therefore factors through the homomorphism $\pi_{1}\left(\mathcal{M}_{1,2}, x_{o}\right) \rightarrow \mathrm{SL}_{2}(\mathbb{Q})$. The universal mapping property of relative completion implies that this induces an action $\widetilde{\mathcal{G}} \rightarrow$ Aut $\mathfrak{p}$ and the corresponding Lie algebra homomorphism $\tilde{\mathfrak{g}} \rightarrow$ Der $\mathfrak{p}$. The composite $\mathfrak{p} \rightarrow \tilde{\mathfrak{g}} \rightarrow$ Der $\mathfrak{p}$ is the homomorphism induced by the conjugation action of $\pi_{1}\left(E^{\prime}, x\right)^{\text {un }}$ on itself and is therefore the adjoint action. Since $\mathfrak{p}$ is free of rank $>1$, it has trivial center, which implies that the adjoint action is faithful and that $\mathfrak{p} \rightarrow \tilde{\mathfrak{g}}$ is injective.

3.6. Hodge Theory. Suppose that $X$ is the complement of a normal crossings divisor in a compact Kähler manifold. Suppose that $F=\mathbb{Q}$ or $\mathbb{R}$ and that $\mathbb{V}$ is a polarized variation of $F$-Hodge structure over $X$. Pick a base point $x_{o} \in X{ }^{4}$ Denote the fiber over $\mathbb{V}$ over $x_{o}$ by $V_{o}$. The Zariski closure of the image of the monodromy representation

$$
\rho: \pi_{1}\left(X, x_{o}\right) \rightarrow \operatorname{Aut}\left(V_{o}\right)
$$

is a reductive $F$-group, $[11,4.2 .6]$. Denote it by $R$. Then one has the relative completion $\mathcal{G}$ of $\pi_{1}\left(X, x_{o}\right)$ with respect to $\rho: \pi_{1}\left(X, x_{o}\right) \rightarrow R(F)$.

Theorem $3.8([15])$. The coordinate ring $\mathcal{O}(\mathcal{G})$ is a Hopf algebra in the category of Ind-mixed Hodge structures over $F$. It has the property that $W_{-1} \mathcal{O}(\mathcal{G})=0$ and $W_{0} \mathcal{O}(\mathcal{G})=\mathcal{O}(R)$.

A slightly weaker version of the theorem is stated in terms of Lie algebras. Denote the prounipotent radical of $\mathcal{G}$ by $\mathcal{U}$. Denote their Lie algebras by $\mathfrak{g}$ and $\mathfrak{u}$, and the Lie algebra of $R$ by $\mathfrak{r}$.

Corollary 3.9 ([15]). The Lie algebra $\mathfrak{g}$ is a Lie algebra in the category of promixed Hodge structures over $F$. It has the property that

$$
\mathfrak{g}=W_{0} \mathfrak{g}, \mathfrak{u}=W_{-1} \mathfrak{g}, \text { and } \operatorname{Gr}_{0}^{W} \mathfrak{g} \cong \mathfrak{r} .
$$

\footnotetext{
${ }^{4}$ We also allow tangential base points.
} 
If $\mathbb{V}$ is a PVHS over $X$ with fiber $V_{o}$ over the base point $x_{o}$, then the composite

$$
H^{\bullet}\left(\mathfrak{u}, V_{o}\right)^{R} \rightarrow H^{\bullet}\left(\Gamma, V_{o}\right) \rightarrow H^{\bullet}(X, \mathbb{V})
$$

of (3.3) with the canonical homomorphism is a morphism of MHS. It is an isomorphism in degrees $\leq 1$ and injective in degree 2. When $X$ is an (orbi) curve, it is an isomorphism in all degrees.

The existence of the mixed Hodge structure on $\mathfrak{u}$ in the unipotent case and when $X$ is not necessarily compact is due to Morgan [31] and Hain [15]. The results in this section also hold in the orbifold case.

Example 3.10. The local system $R^{1} f_{*} \mathbb{Q}$ over $\mathcal{M}_{1,1}$ associated to the universal elliptic curve $f: \mathcal{E} \rightarrow \mathcal{M}_{1,1}$ is a polarized variation of Hodge structure of weight 1 . This variation and its pullback to $\mathcal{M}_{1, n}$ will be denoted by $\mathbb{H}$. It has fiber $H^{1}(E)$ over $[E]$. The Zariski closure of the monodromy representation $\pi_{1}\left(\mathcal{M}_{1,1},[E]\right) \rightarrow$ Aut $H^{1}(E)$ is $\mathrm{SL}\left(H_{1}(E)\right)$, which is isomorphic to $\mathrm{SL}_{2}$. Poincaré duality $H_{1}(E) \cong$ $H^{1}(E)(1)$ induces an isomorphism of $\mathbb{H}(1)$ with the local system over $\mathcal{M}_{1,1}$ whose fiber over $[E]$ is $H_{1}(E)$.

The choice of an elliptic curve $E$ and a non-zero point $x$ of $E$ determines compatible base points of $E^{\prime}, \mathcal{M}_{1,1}$ and $\mathcal{M}_{1,2}$. Denote the Lie algebras of the relative completions of $\pi_{1}\left(\mathcal{M}_{1,1},[E]\right)$ and $\pi_{1}\left(\mathcal{M}_{1,2},[E, x]\right)$ by $\mathfrak{g}$ and $\tilde{\mathfrak{g}}$, respectively. Denote the Lie algebra of the unipotent completion of $\pi_{1}\left(E^{\prime}, x\right)$ by $\mathfrak{p}$. The results of this section imply that each has a natural MHS and that the sequence

$$
0 \rightarrow \mathfrak{p} \rightarrow \tilde{\mathfrak{g}} \rightarrow \mathfrak{g} \rightarrow 0
$$

is exact in the category of MHS. The adjoint action of $\tilde{\mathfrak{g}}$ on $\mathfrak{p}$ induces an action

$$
\tilde{\mathfrak{g}} \rightarrow \operatorname{Der} \mathfrak{p}
$$

Since the inclusion $\mathfrak{p} \rightarrow \tilde{\mathfrak{g}}$ is a morphism of MHS, this homomorphism is a morphism of MHS.

Since the functor $\mathrm{Gr}_{W}^{\bullet}$ is exact on the category of MHS, one can study this action by passing to its associated graded action

$$
\operatorname{Gr}_{\bullet}^{W} \tilde{\mathfrak{g}} \rightarrow \operatorname{Der} \mathbb{L}\left(H_{1}(E)\right) .
$$

\section{A Concrete Approach to Relative Completion}

Suppose that $M$ is the orbifold quotient ${ }^{5} \Gamma \backslash X$ of a simply connected manifold $X$ by a discrete group $\Gamma$. We suppose that $\Gamma$ acts properly discontinuously and virtually freely ${ }^{6}$ on $X$. Our main example will be when $X=\mathfrak{h}$ and $\Gamma$ is a finite index subgroup of $\mathrm{SL}_{2}(\mathbb{Z})$.

Suppose that $R$ is a complex (or real) Lie group and that $\left(\mathfrak{u}_{\alpha}\right)_{\alpha}$ is an inverse system of finite dimensional nilpotent Lie algebras in the category of left $R$-modules. Its limit

$$
\mathfrak{u}=\lim _{\alpha} \mathfrak{u}_{\alpha}
$$

is a pronilpotent Lie algebra in the category of $R$-modules. Denote the unipotent Lie group corresponding to $\mathfrak{u}_{\alpha}$ by $U_{\alpha}$. The prounipotent Lie group corresponding to $\mathfrak{u}$ is the inverse limit of the $U_{\alpha}$.

\footnotetext{
${ }^{5}$ For a detailed and elementary description of what this means, see [19, §3].

${ }^{6}$ That is, $\Gamma$ has a finite index subgroup that acts freely on $X$.
} 
The action of $R$ on $\mathfrak{u}$ induces an action of $R$ on $\mathcal{U}$, so we can form the semi-direct product $^{7} R \ltimes \mathcal{U}$. This is the inverse limit

$$
R \ltimes \mathcal{U}={\underset{\alpha}{\alpha}}_{\lim }\left(R \ltimes U_{\alpha}\right)
$$

If $R$ is an algebraic group, then $R \ltimes \mathcal{U}$ is a proalgebraic group.

Suppose that $\rho: \Gamma \rightarrow R$ is a representation. At this stage, we do not assume that $\rho$ has Zariski dense image. The following assertion is easily proved.

Lemma 4.1. Homomorphisms $\hat{\rho}: \Gamma \rightarrow R \ltimes \mathcal{U}$ that lift $\rho$ correspond to functions $F: \Gamma \rightarrow \mathcal{U}$ that satisfy the 1-cocycle condition

$$
F\left(\gamma_{1} \gamma_{2}\right)=F\left(\gamma_{1}\right)\left(\gamma_{1} \cdot F\left(\gamma_{2}\right)\right) .
$$

The homomorphism $\hat{\rho}$ corresponds to the function $\Gamma \rightarrow \mathcal{U} \times R$

$$
\gamma \mapsto(F(u), \rho(\gamma))
$$

under the identification of $R \ltimes \mathcal{U}$ with $\mathcal{U} \times R$.

Cocycles can be constructed from $\Gamma$-invariant 1 -forms on $X$ with values in $\mathfrak{u}$. Define

$$
E^{\bullet}(X) \hat{\otimes} \mathfrak{u}:=\lim _{\alpha} E^{\bullet}(X) \otimes \mathfrak{u}_{\alpha},
$$

where $E^{\bullet}(X)$ denotes the complex of smooth $\mathbb{C}$-valued forms on $X$. The group $\Gamma$ acts on $E^{\bullet}(X) \hat{\otimes} \mathfrak{u}$ by

$$
\gamma \cdot \omega=\left(\left(\gamma^{*}\right)^{-1} \otimes \gamma\right) \omega
$$

Such a form $\omega$ is invariant if

$$
\left(\gamma^{*} \otimes 1\right) \omega=(1 \otimes \gamma) \omega
$$

for all $\gamma \in \Gamma$.

Let $\Gamma$ act on $X \times \mathcal{U}$ diagonally: $\gamma:(u, x) \rightarrow(\gamma u, \gamma x)$. The projection

$$
X \times \mathcal{U} \rightarrow X
$$

is a $\Gamma$-equivariant principal right $\mathcal{U}$-bundle. Its sections correspond to functions $f: X \rightarrow \mathcal{U}$. Each $\omega \in E^{1}(X) \hat{\otimes} \mathfrak{u}$ defines a connection on this bundle invariant under the right $\mathcal{U}$ action via the formula

$$
\nabla f=d f+\omega f
$$

where $f$ is a $\mathcal{U}$-valued function defined locally on $X$. The connection is $\Gamma$-invariant if and only if $\omega$ is $\Gamma$-invariant. It is flat if and only if $\omega$ is integrable:

$$
d \omega+\frac{1}{2}[\omega, \omega]=0 \quad \text { in } E^{2}(X) \hat{\otimes} \mathfrak{u} .
$$

In this case, parallel transport defines a function

$$
T: P X \rightarrow \mathcal{U}
$$

\footnotetext{
${ }^{7}$ To be clear, the group $R \ltimes U$ is the set $U \times R$ with multiplication

$$
\left(u_{1}, r_{1}\right)\left(u_{2}, r_{2}\right)=\left(u_{1}\left(r_{1} \cdot u_{2}\right), r_{1} r_{2}\right),
$$
}

where $r \cdot u$ denotes the action of $R$ on $U$. We will omit the dot when it is clear from the context that $r u$ means the action of $R$ on $U$. 
from the path space of $X$ into $\mathcal{U}$. With our conventions, this satisfies $T(\alpha * \beta)=$ $T(\beta) T(\alpha)$. When $\omega$ is integrable, $T(\alpha)$ depends only on the homotopy class of $\gamma$ relative to its endpoints. Chen's transport formula implies that the inverse transport function is given by the formula

$$
T(\alpha)^{-1}=1+\int_{\alpha} \omega+\int_{\alpha} \omega \omega+\int_{\alpha} \omega \omega \omega+\cdots
$$

Cf. [20, Cor. 5.6].

Fix a point $x_{o} \in X$. Since $X$ is simply connected, for each $\gamma \in \Gamma$ there is a unique homotopy class $c_{\gamma}$ of paths from $x_{o}$ to $\gamma \cdot x_{o}$.

Proposition 4.3. If $\omega \in E^{1}(X) \hat{\otimes} \mathfrak{u}$ is $\Gamma$-invariant and integrable, then the function $\Theta_{x_{o}}: \Gamma \rightarrow \mathcal{U}$ defined by

$$
\Theta_{x_{o}}(\gamma)=T\left(c_{\gamma}\right)^{-1}
$$

is a well-defined (left) 1-cocycle with values in $\mathcal{U}$ :

$$
\Theta_{x_{o}}(\gamma \mu)=\Theta_{x_{o}}(\gamma)\left(\gamma \cdot \Theta_{x_{o}}(\mu)\right) .
$$

Consequently, the function $\tilde{\rho}_{x_{o}}: \Gamma \rightarrow R \ltimes \mathcal{U}$ defined by $\gamma \mapsto\left(\Theta_{x_{o}}(\gamma), \rho(\gamma)\right)$ is a homomorphism.

Proof. This follows directly from the fact that $c_{\gamma \mu}=c_{\gamma} *\left(\gamma \cdot c_{\mu}\right)$ and the transport formula above.

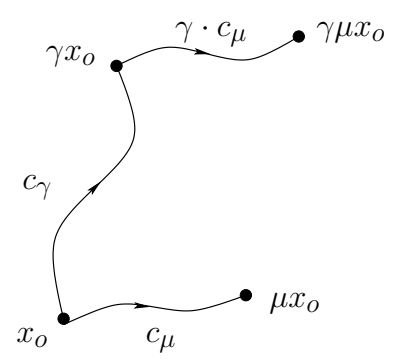

FiguRE 1. The cocycle relation $c_{\gamma \mu}=c_{\gamma} *\left(\gamma \cdot c_{\mu}\right)$

Remark 4.4. The dependence of $\Theta_{x_{o}}$ and $\tilde{\rho}_{x_{o}}$ on $x_{o}$ is easily determined. Suppose that $x^{\prime}$ is a second base point. If $e$ is the unique homotopy class of paths in $X$ from $x_{o}$ to $x^{\prime}$, then $c_{\gamma}^{\prime}:=e^{-1} * c_{\gamma} *(\gamma \cdot e)$ is the unique homotopy class of paths in $X$ from $x^{\prime}$ to $\gamma \cdot x^{\prime}$. Thus

$$
T\left(c_{\gamma}^{\prime}\right)^{-1}=T(e) T\left(c_{\gamma}\right)^{-1}\left(\gamma \cdot T(e)^{-1}\right) .
$$

Since, for $u, v \in \mathcal{U}$ and $r \in R$,

$$
(v, 1)(u, r)\left(v^{-1}, 1\right)=\left(v\left(r \cdot v^{-1}\right), \gamma\right)
$$

in $R \ltimes \mathcal{U}$, the previous formula implies that $\tilde{\rho}_{x^{\prime}}$ is obtained from $\tilde{\rho}_{x_{o}}$ by conjugation by $T(e) \in \mathcal{U}$ and that

$$
\Theta_{x^{\prime}}(\gamma)=T(e) \Theta_{x_{o}}\left(\gamma \cdot T(e)^{-1}\right)
$$


4.1. Variant. Later we will sometimes need a slightly more general setup. This material is standard. More details can be found in $[20, \S 5]$. As above, $R$ acts on $\mathcal{U}$ on the left.

Recall that a factor of automorphy is a smooth function

$$
M: \Gamma \times X \rightarrow R, \quad(\gamma, x) \mapsto M_{\gamma}(x)
$$

that satisfies $M_{\gamma \mu}(x)=M_{\gamma}(\mu x) M_{\mu}(x)$ for all $x \in X$ and $\gamma, \mu \in \Gamma$. One checks easily that the function

$$
\gamma:(x, u) \mapsto\left(\gamma x, M_{\gamma}(x) u\right)
$$

defines a left action of $\Gamma$ on the right principal $\mathcal{U}$ bundle $X \times \mathcal{U}$.

Suppose that $\nabla_{0}$ is a connection on the bundle $X \times \mathcal{U} \rightarrow X$. A 1-form $\omega \in$ $E^{1}(X) \hat{\otimes} \mathfrak{u}$ defines a right $\mathcal{U}$-invariant connection $\nabla$ on this bundle by

$$
\nabla f=\nabla_{0} f+\omega f
$$

where $f: X \rightarrow \mathcal{U}$ is a section. This connection is $\Gamma$-invariant if and only if

$$
\left(\gamma^{*} \otimes 1\right) \omega=\left(1 \otimes M_{\gamma}\right) \omega-\left(\nabla_{0} M_{\gamma}\right) M_{\gamma}^{-1} \text { all } \gamma \in \Gamma
$$

and flat if and only if $\nabla_{0} \omega+[\omega, \omega] / 2=0$.

When $\nabla$ is a flat $\Gamma$-invariant connection, the monodromy representation

$$
\Theta_{x_{o}}: \Gamma \rightarrow R \ltimes \mathcal{U}
$$

is given by

$$
\Theta_{x_{o}}: \gamma \mapsto\left(T\left(c_{\gamma}\right)^{-1}, M_{\gamma}\left(x_{o}\right)\right)
$$

where for a path $\alpha \in X, T(\alpha)$ is given by the formula (4.2).

The setup of the previous section is a special case where $M_{\gamma}(x)=\rho(\gamma)$ and where the connection $\nabla_{0}$ is trivial; that is, $\nabla_{0}=d$.

4.2. A characterization of relative unipotent completion. To give a concrete construction of the completion of $\Gamma$ relative to $\rho: \Gamma \rightarrow R$, we need a useful criterion for when a homomorphism $\Gamma \rightarrow R \ltimes \mathcal{U}$ induces an isomorphism $\mathcal{G} \rightarrow R \ltimes \mathcal{U}$ with the relative completion. In the present situation, the criterion is cohomological.

Suppose that $\omega \in E^{1}(X) \hat{\otimes} \mathfrak{u}$ is an integrable $\Gamma$-invariant 1-form on $X$, as above. Suppose that $V$ is a finite dimensional $R$-module and that $\mathcal{U} \rightarrow$ Aut $V$ is an $R$ invariant homomorphism. ${ }^{8}$ Denote the action of $u \in \mathcal{U}$ on $v \in V$ by $u \cdot v$. Let $M$ be the orbifold quotient of $X \times V$ by the diagonal $\Gamma$-action: $\gamma:(x, v) \mapsto(\gamma x, \rho(\gamma) v)$. The formula

$$
\nabla v=d v+\omega \cdot v
$$

defines a flat $\Gamma$-invariant connection on the vector bundle $X \times V \rightarrow X$. The complex

$$
\left(E^{\bullet}(X) \otimes V\right)^{\Gamma}:=\left\{\omega \in E^{\bullet}(X) \otimes V:\left(\gamma^{*} \otimes 1\right) \omega=(1 \otimes \gamma) \omega\right\}
$$

of $\Gamma$-invariant $V$-valued forms on $X$ has differential defined by

$$
\nabla(\eta \otimes v)=d \eta \otimes v+\eta \wedge(\omega \cdot v),
$$

where $\eta \in E^{\bullet}(X)$ and $v \in V$. It computes the cohomology $H^{\bullet}(M, \mathbb{V})$ of the orbifold $M$ with coefficients in the orbifold local system $\mathbb{V}$ of locally constant sections of the flat vector bundle $\Gamma \backslash(X \times V)$ over $M$.

\footnotetext{
${ }^{8}$ Equivalently, $V$ is an $R \ltimes \mathcal{U}$-module.
} 
Lemma 4.5. If $R$ is reductive, each integrable $\Gamma$-invariant 1-form $\omega \in E^{1}(X) \hat{\otimes} \mathfrak{u}$ induces a ring homomorphism

$$
H^{\bullet}(\mathfrak{u}, V)^{R} \rightarrow H^{\bullet}(M, \mathbb{V}) .
$$

Proof. Note that $\Gamma$ acts on $\mathfrak{u}$ on the left by the formula $\gamma: u \mapsto \rho(\gamma) \cdot u$. A 1-form $\omega \in E^{1}(X) \hat{\otimes} \mathfrak{u}$ can be regarded as a function

$$
\theta_{\omega}: \operatorname{Hom}^{\text {cts }}(\mathfrak{u}, \mathbb{C}) \rightarrow E^{1}(X), \quad \varphi \mapsto(1 \otimes \varphi) \omega .
$$

It is $\Gamma$-equivariant if and only if $\omega$ is. The induced algebra homomorphism

$$
\mathcal{C}^{\bullet}(\mathfrak{u})=\Lambda^{\bullet} \operatorname{Hom}^{\text {cts }}(\mathfrak{u}, \mathbb{C}) \rightarrow E^{\bullet}(X)
$$

commutes with differentials if and only if $\omega$ is integrable. So a $\Gamma$-invariant and integrable 1-form $\omega \in E^{1}(X) \hat{\otimes} \mathfrak{u}$ induces a dga homomorphism

$$
\mathcal{C}^{\bullet}(\mathfrak{u}, V)^{R}=\left[\left(\Lambda^{\bullet} \operatorname{Hom}^{\text {cts }}(\mathfrak{u}, \mathbb{C})\right) \otimes V\right]^{R} \rightarrow\left(E^{\bullet}(X) \otimes V\right)^{\Gamma} .
$$

Since $R$ is reductive, the natural map

$$
H^{\bullet}\left(\mathcal{C}^{\bullet}(\mathfrak{u}, V)^{R}\right) \rightarrow H^{\bullet}\left(\mathcal{C}^{\bullet}(\mathfrak{u}, V)\right)^{R}
$$

is an isomorphism. The result follows.

The desired characterization of relative completion is:

Proposition 4.6. If $R$ is reductive and $\rho: \Gamma \rightarrow R$ has Zariski dense image, then the homomorphism $\tilde{\rho}_{x_{o}}: \Gamma \rightarrow R \ltimes \mathcal{U}$ constructed from $\omega \in\left(E^{1}(X) \hat{\otimes} \mathfrak{u}\right)^{\Gamma}$ above is the completion of $\Gamma$ with respect to $\rho$ if and only if the homomorphism

$$
\theta_{\omega}^{*}:\left[H^{j}(\mathfrak{u}) \otimes V\right]^{R} \rightarrow H^{j}(M, \mathbb{V})
$$

induced by $\theta_{\omega}$ is an isomorphism when $j=0,1$ and injective when $j=2$ for all $R$-modules $V$.

When $X$ is a universal covering of a manifold, such universal 1-forms can be constructed using a suitable modification of Chen's method of power series connections [7].

Proof. Denote the completion of $\Gamma$ relative to $\rho$ by $\mathcal{G}$. The universal mapping property of relative completion implies that the homomorphism $\tilde{\rho}_{x_{o}}: \Gamma \rightarrow R \ltimes \mathcal{U}$ induces a homomorphism $\Psi: \mathcal{G} \rightarrow R \ltimes \mathcal{U}$ that commutes with the projections to $R$. Denote the prounipotent radical of $\mathcal{G}$ by $\mathcal{N}$ and its Lie algebra by $\mathfrak{n}$. Then $\Psi$ induces a homomorphism $\mathcal{N} \rightarrow \mathcal{U}$ and an $R$-invariant homomorphism

$$
\Psi^{*}: H^{\bullet}(\mathfrak{u}) \rightarrow H^{\bullet}(\mathfrak{n}) .
$$

Then for each finite dimensional $R$-module $V$, one has the commutative diagram

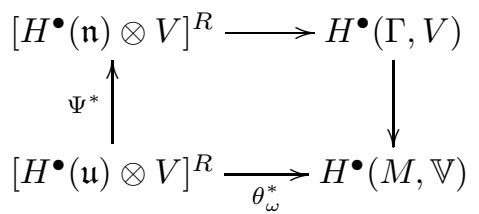

where the right-hand vertical mapping is induced by the orbifold morphism $M \rightarrow$ $B \Gamma$ of $M$ into the classifying space of $\Gamma$. Standard topology implies that this is an isomorphism in degrees 0 and 1 and injective in degree 2. Results in Section 3.2 imply that the top row is an isomorphism in degrees 0 and 1 , and injective in degree 
2. The assumption implies that the left hand vertical map is an isomorphism in degrees 0 and 1 and injective in degree 2 . Since $H^{\bullet}(\mathfrak{u})$ and $H^{\bullet}(\mathfrak{n})$ are direct limits of finite dimensional $R$-modules, by letting $V$ run through all finite dimensional irreducible $R$-modules, we see that $H^{\bullet}(\mathfrak{u}) \rightarrow H^{\bullet}(\mathfrak{n})$ is an isomorphism in degrees 0 and 1 and and injective in degree 2. Proposition 2.1 implies that $\mathfrak{n} \rightarrow \mathfrak{u}$ is an isomorphism. This implies that $\Psi$ is an isomorphism.

4.3. Rational structure. To construct a MHS on the completion of (say) a modular group $\Gamma$, we will first construct its complex form together with its Hodge and weight filtrations using an integrable, $\Gamma$-invariant 1 -form, as above. An easy formal argument, given below, implies that this relative completion has a natural $\mathbb{Q}$ structure provided that $R$ and $\rho$ are defined over $\mathbb{Q}$. To understand the MHS on $\mathcal{O}(\mathcal{G})$, we will need a concrete description of this $\mathbb{Q}$-structure on $R \ltimes \mathcal{U}$ in terms of periods. Explaining this is the goal of this section.

In general, we are not distinguishing between a proalgebraic $F$-group and its group of $F$ rational points. Here, since we are discussing Hodge theory, we will distinguish between a $\mathbb{Q}$-group, and its groups of $\mathbb{Q}$ and $\mathbb{C}$ rational points.

Suppose that $R$ is a reductive group that is defined over $\mathbb{Q}$ and that $\rho: \Gamma \rightarrow R$ takes values in the $\mathbb{Q}$-rational points $R(\mathbb{Q})$ of $R$. Denote the completion of $\Gamma$ with respect to $\rho$ over $\mathbb{Q}$ by $\mathcal{G}$ and its prounipotent radical by $\mathcal{N}$. These are proalgebraic $\mathbb{Q}$-groups. We also have the completion $\mathcal{G}_{\mathbb{C}}$ of $\Gamma$ over $\mathbb{C}$ relative to $\rho$, where the coefficient field is $\mathbb{C}$. Base change (cf. Section 3.3) implies that the natural homomorphism $\mathcal{G} \otimes_{\mathbb{Q}} \mathbb{C} \rightarrow \mathcal{G}_{\mathbb{C}}$ is an isomorphism.

When the hypotheses of Proposition 4.6 are satisfied we obtain a canonical $\mathbb{Q}$ structure on $R \ltimes \mathcal{U}$ from the isomorphism

$$
\psi: \mathcal{G} \otimes_{\mathbb{Q}} \mathbb{C} \stackrel{\simeq}{\longrightarrow} R \ltimes \mathcal{U} .
$$

induced by $\tilde{\rho}_{x_{o}}$. The $\mathbb{Q}$-structure on $\mathcal{U}$ is the image of the restriction $\mathcal{N} \otimes_{\mathbb{Q}} \mathbb{C} \rightarrow \mathcal{U}$ of this isomorphism to $\mathcal{N}$. This induces a canonical $\mathbb{Q}$-structure on $\mathfrak{u}$ via the isomorphism $\mathfrak{u} \cong \mathfrak{n} \otimes_{\mathbb{Q}} \mathbb{C}$.

Proposition 4.7. The canonical $\mathbb{Q}$-structure on $\mathfrak{u}$ is the $\mathbb{Q}$-Lie subalgebra of $\mathfrak{u}$ generated by the set $\left\{\log \Theta_{x_{o}}(\gamma): \gamma \in \Gamma\right\}$.

Proof. Fix a $\mathbb{Q}$-splitting $s$ of the surjection $\mathcal{G} \rightarrow R_{\mathbb{Q}}$. This gives an identification of $\mathcal{G}$ with $R_{\mathbb{Q}} \ltimes \mathcal{N}$. Levi's Theorem (Prop. 3.1) implies that there is a $u \in \mathcal{U}$ such that the composition of

$$
R_{\mathbb{Q}} \stackrel{s}{\longrightarrow} \mathcal{G} \stackrel{\psi}{\longrightarrow} R \ltimes \mathcal{U}
$$

is conjugated to the section $R_{\mathbb{Q}} \rightarrow R \ltimes \mathcal{U}$ that takes $r$ to $(1, r)$ by $u$. That is, the first section takes $r \in R$ to $\left(u(r \cdot u)^{-1}, r\right) \in R \ltimes \mathcal{U}$. The composite

$$
R_{\mathbb{Q}} \ltimes \mathcal{N} \stackrel{\simeq}{\longrightarrow} \mathcal{G} \mathcal{G}_{\mathbb{C}} \stackrel{\simeq}{\longrightarrow} R \ltimes \mathcal{U}
$$

is thus given by the formula

$$
(n, r) \mapsto(\psi(n), 1)\left(u(r \cdot u)^{-1}, r\right)=\left(\psi(n) u(r \cdot u)^{-1}, r\right) \in R \ltimes \mathcal{U} .
$$

The composite

$$
\Gamma \longrightarrow \mathcal{G}(\mathbb{Q}) \stackrel{\simeq}{\longrightarrow} R(\mathbb{Q}) \ltimes \mathcal{N}(\mathbb{Q})
$$


takes $\gamma \in \Gamma$ to $(F(\gamma), \gamma) \in \mathcal{N}(\mathbb{Q}) \times R(\mathbb{Q})$ for some 1-cocycle $F: \Gamma \rightarrow \mathcal{N}(\mathbb{Q})$. Note that every divisible subgroup of the rational points of a prounipotent $\mathbb{Q}$-group $N$ is the set of $\mathbb{Q}$-rational points of a $\mathbb{Q}$-subgroup of $N$. This implies that the smallest subgroup of $\mathcal{N}(\mathbb{Q})$ that contains $\{F(\gamma): \gamma \in \Gamma\}$ is the set of $\mathbb{Q}$-points of a $\mathbb{Q}$-subgroup $\mathcal{S}$ of $\mathcal{N}$. The subgroup $\mathcal{S}$ must be $\mathcal{N}$. This is because the cocycle condition implies that it is a $\Gamma$-invariant subgroup:

$$
\gamma_{1} \cdot F\left(\gamma_{2}\right)=F\left(\gamma_{1}\right)^{-1} F\left(\gamma_{1} \gamma_{2}\right) \in \mathcal{S}(\mathbb{Q}) .
$$

Since $\Gamma$ is Zariski dense in $\mathcal{G}, \mathcal{S}$ is a normal subgroup of $\mathcal{G}$. This implies that $R_{\mathbb{Q}} \ltimes \mathcal{S}$ is a subgroup of $\mathcal{G}=R_{\mathbb{Q}} \ltimes \mathcal{N}$ that contains the image of the canonical homomorphism $\tilde{\rho}: \Gamma \rightarrow \mathcal{G}(\mathbb{Q})$. But since $\tilde{\rho}$ is Zariski dense, we must have $\mathcal{G}=R_{\mathbb{Q}} \ltimes \mathcal{S}$.

Formula (4.8) now implies that the image of $\mathcal{N}(\mathbb{Q})$ in $\mathcal{U}(\mathbb{C})$ is the smallest divisible subgroup of $\mathcal{U}(\mathbb{C})$ that contains the set $\{\psi \circ F(\gamma): \gamma \in \Gamma\}$. But the formula (4.8) and the commutativity of the diagram

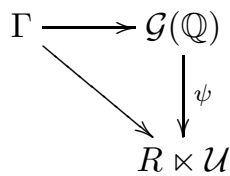

imply that $\psi \circ F=\Theta_{x_{o}}$, so that the image of $\mathcal{N}(\mathbb{Q})$ in $\mathcal{U}$ is the smallest divisible subgroup of $\mathcal{U}$ that contains the set $\left\{\Theta_{x_{o}}(\gamma): \gamma \in \Gamma\right\}$. The result now follows from the Baker-Campbell-Hausdorff formula.

Remark 4.9. One might think that this $\mathbb{Q}$ structure on $\mathcal{U}$ can be constructed as the image of the unipotent completion over $\mathbb{Q}$ of $\operatorname{ker} \rho$ in $\mathcal{U}$. This often works, but it does not when $\Gamma$ is a modular group as $\rho: \Gamma \rightarrow R$ is injective and $\mathcal{U}$ is non-trivial in this case.

4.3.1. Complements. The coordinate $\operatorname{ring} \mathcal{O}(\mathcal{G})$ of $\mathcal{G}$ is isomorphic, as a ring, to $\mathcal{O}(R) \otimes \mathcal{O}(\mathcal{U})$. Its coproduct is twisted by the action of $R$ on $\mathcal{U}$. The $\mathbb{Q}$-form of $\mathcal{O}(\mathcal{G})$ consists of those elements of $\mathcal{O}(R) \otimes \mathcal{O}(\mathcal{U})$ that take rational values on the image of $\Gamma \rightarrow R \ltimes \mathcal{U}$. Since the exponential map $\mathfrak{u} \rightarrow \mathcal{U}$ is an isomorphism of affine schemes, the coordinate ring of $\mathcal{U}$ consists of the polynomial functions on $\mathfrak{u}$ that are continuous functions $\mathfrak{u} \rightarrow \mathbb{C}$. Since the coefficients of the logarithm and exponential functions are rational numbers, $\mathcal{O}\left(\mathcal{U}_{\mathbb{Q}}\right)$ is the ring of continuous polynomials on $\mathfrak{u}_{\mathbb{Q}}$

\section{Relative Completion of Path Torsors}

This section can be omitted on a first reading. Here we consider the relative completion $\mathcal{G}_{x, y}$ of the torsor of paths from $x$ to $y$ in a manifold $M$ with respect to a reductive local system $\mathbb{H}$. This can be described using tannakian formalism. ${ }^{9}$ Here we outline a direct approach partly because it is more concrete and better suits our needs.

We use the setup of the previous section. So $M=\Gamma \backslash X$ where $X$ is a simply connected manifold, $\Gamma$ is a discrete group that acts properly discontinuously and

\footnotetext{
${ }^{9}$ The category of local systems of finite dimensional $F$-vector spaces over $M$ that admit a filtration whose graded quotients are local systems that correspond to representations of the Zariski closure of $\Gamma \rightarrow$ Aut $H_{x}$ is tannakian. The completion $\mathcal{G}_{x, y}$ of the torsor $\Pi(M ; x, y)$ of paths in $M$ from $x$ to $y$ is the torsor of isomorphisms between the fiber functors at $x$ and $y$. It is an affine scheme over $F$.
} 
virtually freely on $X$, and $\rho: \Gamma \rightarrow R$ is a representation of $\Gamma$ into an affine $F$-group $(F=\mathbb{R}$ or $\mathbb{C})$, not yet assumed to be reductive or Zariski dense.

If $M$ is a manifold and $x, y \in M$, then $\Pi(M ; x, y)$ denotes the set of homotopy classes of paths from $x$ to $y$. We need to define what we mean by $\Pi(M ; x, y)$ when the action of $\Gamma$ on $X$ is not free, in which case, $M$ is an orbifold, but not a manifold. Choose a fundamental domain $D$ for the action of $\Gamma$ on $X$. The orbit of each $x \in M$ contains a unique point $\tilde{x} \in D$. Suppose that $x, y \in M$. Elements of $\Pi(M ; x, y)$ can be represented by pairs $\left(\gamma, c_{\gamma}\right)$, where $\gamma \in \Gamma$ and $c_{\gamma}$ is a homotopy class of paths from $\tilde{x}$ to $\gamma \tilde{y}$. (This homotopy class is unique as $X$ is simply connected.) The composition map

$$
\Pi(M ; x, y) \times \Pi(M ; y, z) \rightarrow \Pi(M ; x, z)
$$

is given by

$$
\left(\left(\gamma, c_{\gamma}\right),\left(\mu, c_{\mu}\right)\right) \mapsto\left(\gamma \mu, c_{\gamma \mu}\right):=\left(\gamma \mu, c_{\gamma} *\left(\gamma \cdot c_{\mu}\right)\right) .
$$

Note that $\Pi(M ; x, y)$ is a torsor under the left action of $\pi_{1}(M, x):=\Pi(M, x, x)$ and a torsor under the right action of $\pi_{1}(M, y)$. This definition of $\Pi(M ; x, y)$ agrees with the standard definition when the action of $\Gamma$ is fixed point free.

Now suppose that $H$ is a finite dimensional vector space over $F=\mathbb{C}$ (or $\mathbb{R}$ ), that $R$ is a reductive subgroup of $\mathrm{GL}(H)$, and $\rho: \Gamma \rightarrow R(F)=R$ is a Zariski dense representation. Let $\mathbb{H}$ be the corresponding (orbifold) local system over the orbifold $M$.

As in the previous section, we suppose that $\omega \in E^{1}(X) \hat{\otimes} \mathfrak{u}$ is an integrable, $\Gamma$-invariant 1-form on $X$. Define

$$
\Theta_{x, y}: \Pi(M ; x, y) \rightarrow R \ltimes \mathcal{U} \text { by }\left(\gamma, c_{\gamma}\right) \mapsto\left(\rho(\gamma), T\left(c_{\gamma}\right)^{-1}\right) .
$$

Note that, unless $x=y$, this is not a group homomorphism. The universal mapping property of $\mathcal{G}_{x, y}$ implies that $\Theta_{x, y}$ induces a morphism $\mathcal{G}_{x, y} \rightarrow R \ltimes \mathcal{U}$ of affine schemes such that the diagram

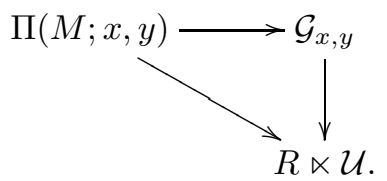

If $\omega$ satisfies the assumptions of Proposition 4.6, then the vertical morphism is an isomorphism. This follows as, in this case, $\mathcal{G}_{x, y}$ and $R \ltimes \mathcal{U}$ are both torsors under the left action of the relative completion $\mathcal{G}_{x, x} \cong R \ltimes \mathcal{U}$ of $\Gamma \cong \pi_{1}(M, x)$ with respect to $\rho$.

If $R$ and $\rho$ are defined over $\mathbb{Q}$, then $\mathcal{G}_{x, y}$ has a natural $\mathbb{Q}$ structure consisting of those elements of $\mathcal{O}\left(\mathcal{G}_{x, y}\right)$ that take rational values on the image of $\Pi(M ; x, y)$ in $\mathcal{G}_{x, y}$.

\section{Zucker's Mixed Hodge Complex}

In this section we recall the construction of the natural MHS on the cohomology of a smooth curve with coefficients in a polarized variation of Hodge structure.

Suppose that $C$ is a compact Riemann surface and that $D$ is a finite subset, which we assume to be non-empty. Then $C^{\prime}:=C-D$ is a smooth affine curve. Suppose that $\mathbb{V}$ is a polarized variation of Hodge structure over $C^{\prime}$ of weight $m$. For simplicity, we assume that the local monodromy about each $x \in D$ is unipotent. Zucker $[45, \S 13]$ constructs a cohomological Hodge complex $\mathbf{K}(\mathbb{V})$ that computes 
the MHS on $H^{\bullet}\left(C^{\prime}, \mathbb{V}\right)$. In this section we recall the definition of its complex component $\mathbf{K}_{\mathbb{C}}(\mathbb{V})$, together with its Hodge and weight filtrations. We first recall a few basic facts about mixed Hodge complexes.

6.1. Review of mixed Hodge complexes. This is a very brief outline of how one constructs a mixed Hodge structure on a graded vector space using a mixed Hodge complex. Full details can be found in [11].

The standard method for constructing a mixed Hodge structure on a graded invariant $M^{\bullet}$ of a complex algebraic variety is to express the invariant as the cohomology of a mixed Hodge complex (MHC). Very briefly, a MHC K consists of:

(i) two complexes $K_{\mathbb{Q}}^{\bullet}$ and $K_{\mathbb{C}}^{\bullet}$, each endowed with a weight filtration $W_{\bullet}$ by subcomplexes,

(ii) a $W \bullet$ filtered quasi-isomorphism between $K_{\mathbb{Q}} \otimes \mathbb{C}$ and $K_{\mathbb{C}}$.

(iii) a Hodge filtration $F^{\bullet}$ of $K_{\mathbb{C}}^{\bullet}$ by subcomplexes.

These are required to satisfy several technical conditions, which we shall omit, although the lemma below encodes some of them. The complexes $K_{\mathbb{Q}}^{\bullet}$ and $K_{\mathbb{C}}^{\bullet}$ compute the $\mathbb{Q}$ - and $\mathbb{C}$-forms of the invariant $M$ :

$$
M_{\mathbb{Q}}^{\bullet} \cong H^{\bullet}\left(K_{\mathbb{Q}}^{\bullet}\right) \text { and } M_{\mathbb{C}}^{\bullet} \cong H^{\bullet}\left(K_{\mathbb{C}}^{\bullet}\right)
$$

The quasi-isomorphism between them is compatible with these isomorphisms. The weight filtration of $K_{\mathbb{Q}}^{\bullet}$ induces a weight filtration of $M_{\mathbb{Q}}$ by

$$
W_{m} M_{\mathbb{Q}}^{j}=\operatorname{im}\left\{H^{j}\left(W_{m-j} K_{\mathbb{Q}}^{\bullet}\right) \rightarrow M_{\mathbb{Q}}^{j}\right\} .
$$

The assumption that the quasi-isomorphism between $K_{\mathbb{Q}}^{\bullet} \otimes \mathbb{C}$ and $K_{\mathbb{C}}^{\bullet}$ be $W_{\bullet}$-filtered implies that the weight filtrations of $K_{\mathbb{Q}}^{\bullet}$ and $\mathcal{K}_{\mathbb{C}}^{\bullet}$ induce the same weight filtration on $M^{\bullet}$. That is,

$$
\left(W_{m} M_{\mathbb{Q}}^{j}\right) \otimes \mathbb{C}=\operatorname{im}\left\{H^{j}\left(W_{m-j} K_{\mathbb{C}}^{\bullet}\right) \rightarrow M_{\mathbb{C}}^{j}\right\}
$$

Finally, the Hodge filtration of $K_{\mathbb{C}}^{\bullet}$ induces the Hodge filtration of $M_{\mathbb{C}}^{\bullet}$ via

$$
F^{p} M_{\mathbb{C}}^{\bullet}:=\operatorname{im}\left\{H^{\bullet}\left(F^{p} K_{\mathbb{C}}^{\bullet}\right) \rightarrow M_{\mathbb{C}}^{\bullet} .\right.
$$

If $\mathbf{K}$ is a MHC, then $M^{\bullet}$ is a MHS with these Hodge and weight filtrations.

We shall need the following technical statement. As pointed out above, this is equivalent to several of the technical conditions satisfied by a MHC.

Lemma $6.1([14,3.2 .8])$. Suppose that $\left(K_{\mathbb{C}}^{\bullet}, W_{\bullet}, F^{\bullet}\right)$ is the complex part of a $M H C$. If $u \in F^{p} W_{m} K_{\mathbb{C}}^{j}$ is exact in $K_{\mathbb{C}}^{\bullet}$, then there exists $v \in F^{p} W_{m+1} K_{\mathbb{C}}^{j-1}$ such that $d v=u$.

Finally, a cohomological mixed Hodge complex (CMHC) is a collection of filtered complexes of sheaves on a variety (or a topological space) with the property that the global sections of a collection of acyclic resolutions of its components is a MHC. For details, see [11].

6.2. Zucker's cohomological MHC. We'll denote Zucker's cohomological MHC for computing the MHS on $H^{\bullet}\left(C^{\prime}, \mathbb{V}\right)$ by $\mathbf{K}(\mathbb{V})$. We describe only its complex part $\mathbf{K}_{\mathbb{C}}(\mathbb{V})$. When trying to understand the definition of its weight filtration, the reader may find it useful to read the following section on the limit MHS of $\mathbb{V}$ at a tangent vector to a cusp $P \in D$. 
Set $\mathcal{V}=\mathbb{V} \otimes \mathcal{O}_{C^{\prime}}$. This has a canonical flat connection $\nabla$. This extends to a meromorphic connection

$$
\nabla: \overline{\mathcal{V}} \rightarrow \Omega_{C}^{1}(\log D) \otimes \overline{\mathcal{V}}
$$

on Deligne's canonical extension $\overline{\mathcal{V}}$ of it to $C$. Schmid's Nilpotent Orbit Theorem [36] implies that the Hodge sub-bundles of $\mathcal{V}$ extend to holomorphic sub-bundles $F^{p} \overline{\mathcal{V}}$ of $\overline{\mathcal{V}}$.

As a sheaf, $\mathbf{K}_{\mathbb{C}}(\mathbb{V})$ is simply $\Omega_{C}^{\bullet}(\log D) \otimes \overline{\mathcal{V}}$ with the differential $\nabla$. Standard arguments imply that $H^{\bullet}\left(C, \mathbf{K}_{\mathbb{C}}(\mathbb{V})\right)$ is isomorphic to $H^{\bullet}\left(C^{\prime}, \mathbb{V}\right)$. Its Hodge filtration is defined in the obvious way:

$$
F^{p} \mathbf{K}_{\mathbb{C}}(\mathbb{V}):=\sum_{s+t=p}\left(F^{s} \Omega_{C}^{\bullet}(\log D)\right) \otimes F^{t} \overline{\mathcal{V}} .
$$

In degree 0 the weight filtration is simply

$$
0=W_{m-1} \mathbf{K}_{\mathbb{C}}^{0}(\mathbb{V}) \subseteq W_{m} \mathbf{K}_{\mathbb{C}}^{0}(\mathbb{V})=\mathbf{K}_{\mathbb{C}}^{0}(\mathbb{V}) .
$$

In degree $1, W_{r} \mathbf{K}_{\mathbb{C}}^{1}(\mathbb{V})$ vanishes when $r<m$. To define the remaining terms in degree 1 , consider the reside mapping

$$
\operatorname{Res}_{P}: \Omega_{C}^{1}(\log D) \otimes \overline{\mathcal{V}} \rightarrow V_{P}
$$

at $P \in C$, which takes values in the fiber $V_{P}$ of $\overline{\mathcal{V}}$ over $P$. The residue $N_{P}$ of the connection $\nabla$ on $\overline{\mathcal{V}}$ at $P \in C$ is the local monodromy logarithm divided by $2 \pi i$. It acts on $V_{P}$. When $r \geq 0$, the stalk of $W_{m+r} \mathbf{K}_{\mathbb{C}}^{1}(\mathbb{V})$ at $P$ is

$$
W_{m+r} \mathbf{K}_{\mathbb{C}}^{1}(\mathbb{V})_{P}:=\operatorname{Res}_{P}^{-1}\left(\operatorname{im} N_{P}+\operatorname{ker} N_{P}^{r}\right) .
$$

Note that, when $P \notin D$, the reside map vanishes (and so does $N_{P}$ ), so that the stalk of $W_{m} \mathbf{K}_{\mathbb{C}}^{1}(\mathbb{V})$ when $P \notin D$ is $\mathbf{K}_{\mathbb{C}}(\mathbb{V})_{P}$.

The Hodge and weight filtrations on $H^{j}\left(C^{\prime}, \mathbb{V}\right)$ are defined by

$$
F^{p} H^{j}\left(C^{\prime}, \mathbb{V}\right)=\operatorname{im}\left\{H^{j}\left(C, F^{p} \mathbf{K}_{\mathbb{C}}(\mathbb{V})\right) \rightarrow H^{j}\left(C^{\prime}, \mathbb{V}\right)\right\}
$$

and

$$
W_{m} H^{j}\left(C^{\prime}, \mathbb{V}\right)=\operatorname{im}\left\{H^{j}\left(C, W_{m-j} \mathbf{K}_{\mathbb{C}}(\mathbb{V})\right) \rightarrow H^{j}\left(C^{\prime}, \mathbb{V}\right)\right\} .
$$

The definition of the weight filtration implies that $H^{0}\left(C^{\prime}, \mathbb{V}\right)$ has weight $m$ and that the weights on $H^{1}\left(C^{\prime}, \mathbb{V}\right)$ are $\geq 1+m$.

Remark 6.2. Let $j: C^{\prime} \rightarrow C$ denote the inclusion. The complex of sheaves $W_{m} \mathbf{K}_{\mathbb{C}}(\mathbb{V})$ on $C$ is a cohomological Hodge complex that is easily seen to be quasiisomorphic to the sheaf $j_{*} \mathbb{V}$ on $C$. It therefore computes the intersection cohomology $I H^{j}(C, \mathbb{V})$ and shows that it has a canonical pure Hodge structure of weight $m+j$. For more details, see Zucker's paper [45].

6.3. The limit MHS on $V_{P}$. Suppose that $P \in D$. For each choice of a non-zero tangent vector $\vec{v} \in T_{P} C$ there is a limit MHS, denoted $V_{\vec{v}}$ on $V_{P}$. The $p$ th term of the Hodge filtration is the fiber of $F^{p} \overline{\mathcal{V}}$ over $P$. The weight filtration is the monodromy weight filtration shifted so that its average weight is $m$, the weight of $\mathbb{V}$. The $\mathbb{Q}$ (or $\mathbb{Z}$ structure, if that makes sense) is constructed by first choosing a local holomorphic coordinate $t$ defined on a disk $\Delta$ containing $P$ where $t(P)=0$. Assume that $\Delta \cap D=\{P\}$. Standard ODE theory (cf. [44, Chapt. II]) implies that there is a trivialization $\Delta \times V_{P}$ of the restriction of $\overline{\mathcal{V}}$ to $\Delta$ such that the connection $\nabla$ is given by

$$
\nabla f=d f+N_{P}(f) \frac{d t}{t}
$$


with respect to this trivialization, where $f: \Delta \rightarrow V_{P}$. Suppose that $Q \in \Delta-$ $\{P\}$. The $\mathbb{Q}$-structure on $V_{P}$ corresponding to the tangent vector $\vec{v}=t(Q) \partial / \partial t$ is obtained from the $\mathbb{Q}$ structure $V_{Q, \mathbb{Q}}$ on $V_{Q}$ by identifying $V_{P}$ with $V_{Q}$ via the trivialization. This MHS depends only on the tangent vector and not on the choice of the holomorphic coordinate $t$.

For all non-zero $\vec{v} \in T_{P} C$, the monodromy logarithm $N_{P}: V_{\vec{v}} \rightarrow V_{\vec{v}}$ acts as a morphism of type $(-1,-1)$. This implies that $V_{\vec{v}} / \operatorname{im} N_{P}$ has a natural MHS for all $\vec{v} \neq 0$. Since $N_{P}$ acts trivially on this, the MHS on $V_{P} / \operatorname{im} N_{P}$ has a natural MHS that is independent of the choice of the tangent vector $\vec{v} \in T_{P} C$. The definition of the weight filtration on $V_{\vec{v}}$ implies that the weight filtration on $V_{P} /$ im $N_{P}$ is

$$
W_{m+r}\left(V_{P} / \operatorname{im} N_{P}\right)=\left(\operatorname{im} N_{P}+\operatorname{ker} N_{P}^{r-1}\right) / \operatorname{im} N_{P}
$$

when $r \geq 0$ and $W_{m+r}\left(V_{P} / \operatorname{im} N_{P}\right)=0$ when $r<0$.

There is a canonical isomorphism

$$
H^{1}(\Delta-\{P\}, \mathbb{V}) \cong V_{P} / \operatorname{im} N_{P} .
$$

from which it follows that this cohomology group has a canonical MHS for each $P \in D$.

6.4. An exact sequence. Observe that

$$
\mathbf{K}_{\mathbb{C}}(\mathbb{V}) / W_{m} \mathbf{K}_{\mathbb{C}}(\mathbb{V})=\bigoplus_{P \in D} i_{P *}\left(V_{P} / N_{P} V_{P}\right)(-1)[-1] .
$$

This and the exact sequence of sheaves

$$
0 \rightarrow W_{m} \mathbf{K}(\mathbb{V}) \rightarrow \mathbf{K}(\mathbb{V}) \rightarrow \mathbf{K}(\mathbb{V}) / W_{m} \rightarrow 0
$$

on $C$ gives the exact sequence of MHS

$$
\begin{aligned}
0 \rightarrow W_{m+1} H^{1}\left(C^{\prime}, \mathbb{V}\right) \rightarrow H^{1}\left(C^{\prime}, \mathbb{V}\right) & \\
& \rightarrow \bigoplus_{P \in D}\left(V_{P} / \operatorname{im} N_{P}\right)(-1) \rightarrow I H^{2}(C, \mathbb{V}) \rightarrow 0 .
\end{aligned}
$$

Here we are assuming that we are in the "interesting case" where $D$ is non-empty.

Since $H^{0}\left(C^{\prime}, \mathbb{V}\right)=I H^{0}(C, \mathbb{V})$, and since this is dual to $I H^{2}(C, \mathbb{V})$, we see that the sequence

$$
0 \rightarrow W_{m+1} H^{1}\left(C^{\prime}, \mathbb{V}\right) \rightarrow H^{1}\left(C^{\prime}, \mathbb{V}\right) \rightarrow \bigoplus_{P \in D}\left(V_{P} / \operatorname{im} N_{P}\right)(-1) \rightarrow 0
$$

is exact when $H^{0}\left(C^{\prime}, \mathbb{V}\right)=0$.

6.5. A MHC of smooth forms. To extend this MHS from the cohomology groups $H^{1}\left(C^{\prime}, \mathbb{V}\right)$ to one on relative completion of its fundamental group, we will need the complex part of a global MHC of smooth forms. The construction of this from $\mathbf{K}_{\mathbb{C}}(\mathbb{V})$ is standard. We recall the construction.

The resolution of $\mathbf{K}_{\mathbb{C}}(\mathbb{V})$ by smooth forms is the total complex of the double complex

$$
\mathcal{K}_{\mathbb{C}}^{\bullet \bullet}(\mathbb{V}):=\mathbf{K}_{\mathbb{C}}(\mathbb{V}) \otimes_{\mathcal{O}_{C}} \mathcal{E}_{C}^{0, \bullet},
$$

where $\mathcal{E}_{C}^{0, \bullet}$ denotes the sheaf of smooth forms on $C$ of type $(0, \bullet)$. The Hodge and weight filtrations extend as

$$
F^{p} \mathcal{K}_{\mathbb{C}}^{\bullet \bullet}(\mathbb{V}):=\mathcal{K}_{\mathbb{C}}^{\geq p, \bullet}(\mathbb{V})=\left(F^{p} \mathbf{K}_{\mathbb{C}}(\mathbb{V})\right) \otimes_{\mathcal{O}_{C}} \mathcal{E}_{C}^{0, \bullet}
$$


and

The global sections

$$
W_{r} \mathcal{K}_{\mathbb{C}}^{\bullet \bullet \bullet}(\mathbb{V}):=\left(W_{r} \mathbf{K}_{\mathbb{C}}(\mathbb{V})\right) \otimes_{\mathcal{O}_{C}} \mathcal{E}_{C}^{0, \bullet}
$$

$$
K^{\bullet}(C, D ; \mathbb{V}):=H^{0}\left(C, \operatorname{tot} \mathcal{K}_{\mathbb{C}}^{\bullet \bullet}(\mathbb{V})\right) .
$$

of (6.4) is a sub dga of $E^{\bullet}\left(C^{\prime}, \mathbb{V}\right)$. It has Hodge and weight filtrations defined by taking the global sections of the Hodge and weight filtrations of (6.4). It is the complex part of a mixed Hodge complex.

The Hodge and weight filtrations on $H^{j}\left(C^{\prime}, \mathbb{V}\right)$ are

$$
F^{p} H^{j}\left(C^{\prime}, \mathbb{V}\right)=\operatorname{im}\left\{H^{j}\left(F^{p} K^{\bullet}(C, D ; \mathbb{V})\right) \rightarrow H^{j}\left(C^{\prime}, \mathbb{V}\right)\right\}
$$

and

$$
W_{m} H^{j}\left(C^{\prime}, \mathbb{V}\right)=\operatorname{im}\left\{H^{j}\left(W_{m-j} K^{\bullet}(C, D ; \mathbb{V})\right) \rightarrow H^{j}\left(C^{\prime}, \mathbb{V}\right)\right\} .
$$

Zucker's MHC and its resolution by smooth forms are natural in the local system $\mathbb{V}$ and are compatible with tensor products: if $\mathbb{V}_{1}, \mathbb{V}_{2}$ and $\mathbb{V}_{3}$ are PVHS over $C^{\prime}$, then a morphism $\mathbb{V}_{1} \otimes \mathbb{V}_{2} \rightarrow \mathbb{V}_{3}$ of PVHS induces morphism

$$
\mathbf{K}\left(\mathbb{V}_{1}\right) \otimes \mathbf{K}\left(\mathbb{V}_{2}\right) \rightarrow \mathbf{K}\left(\mathbb{V}_{1} \otimes \mathbb{V}_{2}\right) \rightarrow \mathbf{K}\left(\mathbb{V}_{3}\right) .
$$

of CMHCs and dga homomorphism

$$
K^{\bullet}\left(C, D ; \mathbb{V}_{1}\right) \otimes K^{\bullet}\left(C, D ; \mathbb{V}_{2}\right) \rightarrow K^{\bullet}\left(C, D ; \mathbb{V}_{1} \otimes \mathbb{V}_{2}\right) \rightarrow K^{\bullet}\left(C, D ; \mathbb{V}_{3}\right)
$$

that preserve the Hodge and weight filtrations.

6.6. Remarks about the orbifold case. Zucker's work extends formally to the orbifold case. For us, an orbi-curve $C^{\prime}=C-D$ is the orbifold quotient of a smooth curve $X^{\prime}=X-E$ by a finite group $G$. This action does not have to be effective. (That is, $G \rightarrow$ Aut $X$ does not have to be injective.) An orbifold variation of MHS $\mathbb{V}$ over $C^{\prime}$ is an admissible variation of MHS $\mathbb{V}_{X}$ over $X^{\prime}$ together with a $G$-action such that the projection $\mathbb{V} \rightarrow X^{\prime}$ is $G$-equivariant. For each $g \in G$, we require that the map $g: \mathbb{V}_{X} \rightarrow \mathbb{V}_{X}$ induce an isomorphism of variations of MHS $g^{*} \mathbb{V}_{X} \cong \mathbb{V}_{X}$.

With these assumptions, $G$ acts on $\mathbf{K}^{\bullet}\left(X, E ; \mathbb{V}_{X}\right)$ and $\mathbf{K}^{\bullet}\left(X, E ; \mathbb{V}_{X}\right)^{G}$ is a sub MHC. Define

$$
\mathbf{K}^{\bullet}(C, D ; \mathbb{V})=\mathbf{K}^{\bullet}\left(X, E ; \mathbb{V}_{X}\right)^{G} .
$$

This computes the cohomology $H^{\bullet}\left(C^{\prime}, \mathbb{V}\right)$ and implies that it has a MHS such that the canonical isomorphism $H^{\bullet}\left(C^{\prime}, \mathbb{V}\right) \cong H^{\bullet}\left(X^{\prime}, \mathbb{V}_{X}\right)^{G}$ is an isomorphism of MHS.

\section{Relative Completion of Fundamental Groups of Affine Curves}

In this section we use the results of the last two sections to construct, under suitable hypotheses, a MHS on the relative completion of the fundamental group of an affine curve. As in the previous section, we suppose that $C$ is a compact Riemann surface and that $D$ is a finite subset of $C$. Here we suppose, in addition, that $D$ is non-empty, so that $C^{\prime}=C-D$ is an affine curve. Suppose that $\mathbb{V}$ is a polarized variation of $\mathbb{Q}$-HS over $C^{\prime}$ with unipotent monodromy about each $P \in D$. Denote the fiber of $\mathbb{V}$ over $x$ by $V_{x}$. The Zariski closure of the monodromy representation

$$
\rho: \pi_{1}\left(C^{\prime}, x\right) \rightarrow \operatorname{Aut}\left(V_{x}\right)
$$

is a reductive $\mathbb{Q}$-group [39, Lem. 2.10], which we will denote by $R_{x}$. Fix a base point $x_{o} \in C$. Set $R=R_{x_{o}}$. Each monodromy group $R_{x}$ is isomorphic to $R$; 
the isomorphism is unique mod inner automorphisms. We thus have Zariski dense monodromy representations

$$
\rho_{x}: \pi_{1}\left(C^{\prime}, x\right) \rightarrow R_{x}(\mathbb{Q})
$$

for each $x \in X$. Denote the completion of $\pi_{1}\left(C^{\prime}, x\right)$ with respect to $\rho_{x}$ by $\mathcal{G}_{x}$, and its Lie algebra by $\mathfrak{g}_{x}$. We will construct natural MHSs on $\mathcal{O}\left(\mathcal{G}_{x}\right)$ and on $\mathfrak{g}_{x}$ that are compatible with their algebraic structures (Hopf algebra, Lie algebra). Before doing this we need to show that the connection form $\Omega$ can be chosen to have coefficients in Zucker's MHC and be compatible with the various Hodge and weight filtrations.

For simplicity, we make the following assumptions:

(i) Every irreducible representation of $R$ is absolutely irreducible. That is, it remains irreducible when we extend scalars from $\mathbb{Q}$ to $\mathbb{C}$.

(ii) For each irreducible representation $V_{\lambda}$ of $R$, the corresponding local system $\mathbb{V}_{\lambda}$ over $C^{\prime}$ underlies a PVHS over $C^{\prime}$. The Theorem of the Fixed Part (stated below) implies that this PVHS is unique up to Tate twist.

These hold in our primary example, where $C^{\prime}$ is a quotient $X_{\Gamma}$ of the upper half plane by a finite index subgroup of $\mathrm{SL}_{2}(\mathbb{Z})$ and $R \cong \mathrm{SL}_{2}$.

7.1. The bundle $\mathfrak{u}$ of Lie algebras. Denote the set of isomorphism classes of irreducible $R$-modules by $\check{R}$. Fix a representative $V_{\lambda}$ of each $\lambda \in \check{R}$ and the structure of a PVHS on the corresponding local system $\mathbb{V}_{\lambda}$ over $C^{\prime}$. Filter

$$
\check{R}_{1} \subset \check{R}_{2} \subset \check{R}_{3} \subset \cdots \subset \bigcup_{n} \check{R}_{n}=\check{R}
$$

$\check{R}$ by finite subsets such that if $\lambda \in \check{R}_{n}$ and $\mu \in \check{R}_{m}$, then the isomorphism class of $V_{\lambda} \otimes V_{\mu}$ is in $\check{R}_{m+n}$. For example, when $R=\mathrm{SL}_{2}$, one can take $\check{R}_{n}$ to be the set of all symmetric powers $S^{m} H$ with $m \leq n$ of the defining representation $H$ of $\mathrm{SL}_{2}$.

For each $\lambda \in \check{R}$, the variation MHS $H^{1}\left(C^{\prime}, \mathbb{V}_{\lambda}\right)^{*} \otimes \mathbb{V}_{\lambda}$, being the tensor product of a constant MHS with a PVHS, is an admissible variation of MHS over $C^{\prime}$. Note that the VMHS structure on it does not change when $\mathbb{V}_{\lambda}$ is replaced by $\mathbb{V}_{\lambda}(n)$, so that the VMHS $H^{1}\left(C^{\prime}, \mathbb{V}_{\lambda}\right)^{*} \otimes \mathbb{V}_{\lambda}$ is independent of the choice of the PVHS structure on $\mathbb{V}_{\lambda}$. Since the weights of $H^{1}\left(C, \mathbb{V}_{\lambda}\right)$ are at least $1+$ the weight of $\mathbb{V}_{\lambda}$, the weights of $H^{1}\left(C^{\prime}, \mathbb{V}_{\lambda}\right)^{*} \otimes \mathbb{V}_{\lambda}$ are $\leq-1$.

The inverse limit

$$
\mathfrak{u}_{1}:=\underbrace{}_{n} \lim _{\lambda \in \breve{R}} H^{1}\left(C^{\prime}, \mathbb{V}_{\lambda}\right)^{*} \otimes \mathbb{V}_{\lambda}
$$

is pro-variation of MHS over $\mathbb{C}$ of negative weight. ${ }^{10}$ That is, $\mathfrak{u}_{1}=W_{-1} \mathfrak{u}_{1}$. Observe that its fiber

$$
\varliminf_{n} \lim _{\lambda \in \check{R}} H^{1}\left(C^{\prime}, \mathbb{V}_{\lambda}\right)^{*} \otimes V_{\lambda, o}=\prod_{\lambda \in \check{R}} H^{1}\left(C^{\prime}, \mathbb{V}_{\lambda}\right)^{*} \otimes V_{\lambda, o}
$$

over $x_{o}$ is the abelianization of the prounipotent radical of the completion of $\pi_{1}\left(C^{\prime}, x_{o}\right)$ with respect to the homomorphism to $R$.

\footnotetext{
${ }^{10}$ Note that this is a very special kind of variation of MHS - it is a direct sum of variations that are the tensor product of a constant MHS with a PVHS. Their asymptotic behaviour is determined by that of the PVHS that occur in the summands.
} 
The degree $n$ part $V \mapsto \mathbb{L}_{n}(V)$ of the free Lie algebra is a Schur functor, so that it makes sense to apply it to bundles. Set

$$
\mathfrak{u}_{n}=\mathbb{L}_{n}\left(\mathfrak{u}_{1}\right):={\underset{\lim }{\hbar}}_{n} \mathbb{L}_{n}\left(\bigoplus_{\lambda \in \check{R}} H^{1}\left(C^{\prime}, \mathbb{V}_{\lambda}\right)^{*} \otimes \mathbb{V}_{\lambda}\right)
$$

and

$$
\mathfrak{u}:=\varliminf_{n} \varliminf_{j=1}^{n} \bigoplus_{j} \mathfrak{u}_{j} \text { and } \mathfrak{u}^{N}:=\lim _{n \geq N} \bigoplus_{j=N}^{n} \mathfrak{u}_{j} .
$$

These are admissible pro-variations of MHS over $C^{\prime}$. Denote the fiber of $\mathfrak{u}$ over $x$ by $\mathfrak{u}_{x}$. It is abstractly isomorphic to the prounipotent radical of the completion of $\pi_{1}\left(C^{\prime}, x\right)$ relative to the monodromy representation $\pi_{1}\left(C^{\prime}, x\right) \rightarrow R$. The fiber of $\mathfrak{u}^{N}$ over $x$ is the $n$th term $L^{n} \mathfrak{u}_{x}$ of the lower central series (LCS) of $\mathfrak{u}_{x}$.

7.2. Some technicalities. The Theorem of the Fixed Part states that if $\mathbb{A}$ is an admissible VMHS over a smooth variety $X$, then $H^{0}(X, \mathbb{A})$ has a natural MHS with the property that for each $x \in X$, the natural inclusion $H^{0}(X, \mathbb{A}) \rightarrow A_{x}$ is a morphism of MHS. In the algebraic case, it is enough to prove this when $X$ is a curve. When $\mathbb{A}$ is pure, this follows from Zucker's MHS [45] on $H^{\bullet}(X, \mathbb{V})$. The general case follows from Saito's theory of Hodge and mixed Hodge modules [34, 35].

The following is a direct consequence of the Theorem of the Fixed Part. Its proof is left as an exercise.

Lemma 7.1. Assume that $\mathbb{A}$ is an admissible VMHS over $C^{\prime}$ whose monodromy representation $\pi_{1}\left(C^{\prime}, x_{o}\right) \rightarrow$ Aut $A_{x_{o}}$ factors through $\pi_{1}\left(C^{\prime}, x_{o}\right) \rightarrow R$. With the assumptions above, each $R$ isotypical component of $\mathbb{A}$ over $C^{\prime}$ is an admissible $V M H S$. If $\mathbb{V}_{\lambda}$ is a PVHS that corresponds to the irreducible $R$-module $V_{\lambda}$, then the natural mapping

$$
\bigoplus_{\lambda \in \check{R}} H^{0}\left(C^{\prime}, \mathbb{V}_{\lambda}\right) \otimes \mathbb{V}_{\lambda} \rightarrow \mathbb{A}
$$

is an isomorphism of admissible VMHS. In particular, the structure of a PVHS on $\mathbb{V}_{\lambda}$ is unique up to Tate twist.

Every pro object of the category of admissible VMHS $\mathbb{A}$ over $C^{\prime}$ is thus of the form

$$
\mathbb{A}=\prod_{\lambda \in \check{R}} \mathbb{V}_{\lambda} \otimes A_{\lambda},
$$

where each $A_{\lambda}$ is a MHS. Define

$$
\mathbf{K}(\mathbb{A})=\prod_{\lambda \in \check{R}} \mathbf{K}\left(\mathbb{V}_{\lambda}\right) \otimes A_{\lambda} .
$$

This is a pro-CMHC. In particular, its complex part

$$
K^{\bullet}(C, D ; \mathbb{A})=\prod_{\lambda \in \check{R}} K^{\bullet}\left(C, D ; \mathbb{V}_{\lambda}\right) \otimes A_{\lambda}
$$

has naturally defined Hodge and weight filtrations; its differential is strict with respect to the Hodge and weight filtrations (cf. Lemma 6.1). In particular, for all $n \geq 1$, the complexes $K^{\bullet}\left(C, D ; \mathfrak{u}_{n}\right)$ have this strictness property. 
7.3. The connection form $\Omega$. Observe that $H^{1}\left(C^{\prime}, H^{1}\left(C^{\prime}, \mathbb{V}_{\lambda}\right)^{*} \otimes \mathbb{V}_{\lambda}\right)$ is naturally isomorphic (as a MHS) to

$$
H^{1}\left(C^{\prime}, \mathbb{V}_{\lambda}\right) \otimes H^{1}\left(C^{\prime}, \mathbb{V}_{\lambda}\right)^{*} \cong \operatorname{Hom}\left(H^{1}\left(C^{\prime}, \mathbb{V}_{\lambda}\right), H^{1}\left(C^{\prime}, \mathbb{V}_{\lambda}\right)\right)
$$

So, for each $\lambda \in \check{R}$, there is an element $\xi_{\lambda} \in F^{0} W_{0} H^{1}\left(C^{\prime}, H^{1}\left(C^{\prime}, \mathbb{V}_{\lambda}\right)^{*} \otimes \mathbb{V}\right)$ that corresponds to the identity mapping $H^{1}\left(C^{\prime}, \mathbb{V}_{\lambda}\right) \rightarrow H^{1}\left(C^{\prime}, \mathbb{V}_{\lambda}\right)$. Lemma 6.1 implies that this is represented by a 1 -form

$$
\omega_{\lambda} \in F^{0} W_{-1} K^{1}\left(C, D ; H^{1}\left(C^{\prime} ; \mathbb{V}_{\lambda}\right)^{*} \otimes \mathbb{V}_{\lambda}\right) .
$$

Set

$$
\Omega_{1}:=\prod_{\lambda \in \check{R}} \omega_{\lambda} \in K^{1}\left(C, D ; \mathfrak{u}_{1}\right) .
$$

Note that $d \Omega_{1}=0$ and that

$$
\frac{1}{2}\left[\Omega_{1}, \Omega_{1}\right] \in F^{0} W_{-2} K^{2}\left(C, D ; \mathfrak{u}_{2}\right) .
$$

Since $C$ is a surface, $\left[\Omega_{1}, \Omega_{1}\right]$ is closed. Since $C^{\prime}$ is not compact, it is exact. Lemma 6.1 implies that we can find $\Xi_{2}$ in $F^{0} W_{-1} K^{1}\left(C, D ; \mathfrak{u}_{2}\right)$ such that $d \Xi_{2}=$ $\frac{1}{2}\left[\Omega_{1}, \Omega_{1}\right]$. Set

$$
\Omega_{2}=\Omega_{1}-\Xi_{2} \in F^{0} W_{-1} K^{1}\left(C, D ; \mathfrak{u}_{1} \oplus \mathfrak{u}_{2}\right) .
$$

Then

$$
d \Omega_{2}+\frac{1}{2}\left[\Omega_{2}, \Omega_{2}\right] \in F^{0} W_{-2} K^{2}\left(C, D ; \mathfrak{u}^{3}\right) .
$$

Its component that lies in $K^{2}\left(C, D ; \mathfrak{u}_{3}\right)$ is closed and thus exact. So it is the exterior derivative of some $\Xi_{3} \in F^{0} W_{-2} K^{1}\left(C, D ; \mathfrak{u}_{3}\right)$. Set

$$
\Omega_{3}=\Omega_{2}-\Xi_{3} \in F^{0} W_{-1} K^{1}\left(C, D ; \mathfrak{u}_{1} \oplus \mathfrak{u}_{2} \oplus \mathfrak{u}_{3}\right) .
$$

Then

$$
d \Omega_{3}+\frac{1}{2}\left[\Omega_{3}, \Omega_{3}\right] \in F^{0} W_{-2} K^{2}\left(C, D ; \mathfrak{u}^{4}\right) .
$$

Continuing this way, we obtain a sequence of elements $\Xi_{n} \in F^{0} W_{-1} K^{1}\left(C, D ; \mathfrak{u}_{n}\right)$ such that for all $N \geq 2$

$$
\Omega_{N}:=\Omega_{1}-\left(\Xi_{2}+\cdots+\Xi_{N}\right) \in F^{0} W_{-1} K^{1}\left(C, D ; \oplus_{n=1}^{N} \mathfrak{u}_{n}\right)
$$

satisfies

$$
d \Omega_{N}+\frac{1}{2}\left[\Omega_{N}, \Omega_{N}\right] \in F^{0} W_{-2} K^{2}\left(C, D ; \mathfrak{u}^{N+1}\right) .
$$

Then the $\mathfrak{u}$-valued 1 -form

$$
\Omega:=\lim _{N} \Omega_{N} \in F^{0} W_{-1} K^{1}(C, D ; \mathfrak{u})
$$

is integrable:

$$
d \Omega+\frac{1}{2}[\Omega, \Omega]=0
$$

To understand the significance of the form $\Omega$, note that the bundle $\mathfrak{u}_{1}$ over $C^{\prime}$, and hence each $\mathfrak{u}_{n}=\mathbb{L}_{n}\left(\mathfrak{u}_{1}\right)$, is a flat bundle over $C^{\prime}$. The monodromy of each factors through the representation $\rho_{x}: \pi_{1}\left(C^{\prime}, x\right) \rightarrow R$. Summing these, we see that for each $N \geq 1$, the bundle

$$
\mathfrak{u} / \mathfrak{u}^{N+1} \cong \mathfrak{u}_{1} \oplus \cdots \oplus \mathfrak{u}_{N}
$$


is flat with monodromy that factors through $\rho_{x}$. Denote the limit of these flat connections by $\nabla_{0}$. Then

$$
\nabla:=\nabla_{0}+\Omega
$$

defines a new connection on the bundle $\boldsymbol{u}$ over $C^{\prime}$ which is flat as $\Omega$ is integrable. Here we view $\mathfrak{u}$ (and hence $\Omega$ ) as acting on each fiber by inner derivations.

The restriction of the filtration

$$
\mathfrak{u}=\mathfrak{u}^{1} \supset \mathfrak{u}^{2} \supset \mathfrak{u}^{3} \supset \cdots
$$

of $\mathfrak{u}$ to each fiber is the lower central series. Note that

$$
\operatorname{Gr}_{\mathrm{LCS}}^{\bullet} \mathfrak{u}:=\mathfrak{u}^{n} / \mathfrak{u}^{n+1}
$$

is naturally isomorphic to $\mathfrak{u}_{n}$.

Lemma 7.4. Each term $\mathfrak{u}^{n}$ of the lower central series filtration of $\mathfrak{u}$ is a flat subbundle of $(\mathfrak{u}, \nabla)$. The induced connection on $\mathrm{Gr}_{\mathrm{LCS}}^{n} \mathfrak{u} \cong \mathfrak{u}_{n}$ is $\nabla_{0}$.

Proof. This follows from the fact that $\Omega$ takes values in $\boldsymbol{u}$ and that the inner derivations act trivially on $\mathrm{Gr}_{\mathrm{LCS}}^{\bullet} \mathfrak{u}$.

7.4. Hodge and weight bundles and their extensions to $C$. The flat connection $\nabla$ on $\mathfrak{u}$ defines a new complex structure as a (pro) holomorphic vector bundle over $C^{\prime}$. To understand it, write $\Omega=\Omega^{\prime}+\Omega^{\prime \prime}$, where $\Omega^{\prime}$ is of type $(1,0)$ and $\Omega^{\prime \prime}$ is of type $(0,1)$. Set

$$
D^{\prime}=\nabla_{0}+\Omega^{\prime} \text { and } D^{\prime \prime}=\bar{\partial}+\Omega^{\prime \prime}
$$

so that $\nabla=D^{\prime}+D^{\prime \prime}$. Then $D^{\prime \prime}$ is a $(0,1)$-valued form taking values in $\mathfrak{u}$. Note that $\left(D^{\prime \prime}\right)^{2}=0$.

Lemma 7.5. A section $s$ of $\mathfrak{u}$ is holomorphic with respect to the complex structure on $\mathfrak{u}$ defined by the flat connection $\nabla$ if and only if $D^{\prime \prime} s=0$.

Proof. Since $D^{\prime \prime}$ is $\mathcal{O}_{C^{\prime}}$ linear, it suffices to show that $D^{\prime \prime} s=0$ when $s$ is a flat local section of $\boldsymbol{u}$. But this follows as $D^{\prime \prime} s$ is the $(0,1)$ component of $\nabla s$, which vanishes as $s$ is flat.

Denote $\mathfrak{u}$ with this complex structure by $\left(\mathfrak{u}, D^{\prime \prime}\right)$. Since all holomorphic sections of $\left(\mathfrak{u}, D^{\prime \prime}\right)$ are $\mathcal{O}$-linear combinations of flat sections, Lemma 7.4 implies: ${ }^{11}$

Lemma 7.6. The lower central series filtration (7.3) of $\mathfrak{u}$ is a filtration by holomorphic sub-bundles. The isomorphism

$$
\operatorname{Gr}_{\mathrm{LCS}}^{n}\left(\mathfrak{u}, D^{\prime \prime}\right) \cong \mathfrak{u}_{n}
$$

is an isomorphism of holomorphic vector bundles.

Denote the canonical extension of $\mathfrak{u}_{1}$ to $C$ by $\overline{\mathfrak{u}}_{1}$. Then, since the local monodromy operators are unipotent, $\overline{\mathfrak{u}}_{n}:=\mathbb{L}_{n}\left(\overline{\mathfrak{u}}_{1}\right)$ is the canonical extension of $\mathfrak{u}_{n}$ to $C$. Define

$$
\overline{\mathfrak{u}}:=\underbrace{}_{n} \varliminf_{j=1} \bigoplus_{j}^{n} \overline{\mathfrak{u}}_{j} \text { and } \overline{\mathfrak{u}}^{N}:=\lim _{n \geq N} \bigoplus_{j=N}^{n} \overline{\mathfrak{u}}_{j} .
$$

Then $\left(\overline{\mathfrak{u}}, \nabla_{0}\right)$ is the canonical extension of $\left(\mathfrak{u}, \nabla_{0}\right)$ to $C$.

\footnotetext{
${ }^{11}$ This also follows from the fact that $\Omega^{\prime \prime}$ is $\mathfrak{u}$-valued thus acts trivially on the graded quotients of the lower central series filtration of $\mathfrak{u}$.
} 
Our next task is to show that $(\overline{\mathfrak{u}}, \nabla)$ is the canonical extension of $(\mathfrak{u}, \nabla)$ to $C$. Since smooth logarithmic $(0,1)$-forms on $C$ with poles on $D$ are smooth on $C$, it follows that $\Omega^{\prime \prime}$ is a smooth, $\overline{\mathfrak{u}}$-valued $(0,1)$-form on $C$. It follows that $D^{\prime \prime}$ extends to a $(0,1)$ form-valued operator on smooth sections of $\overline{\mathfrak{u}}$. Since $\left(D^{\prime \prime}\right)^{2}=0$, it defines a complex structure on $\overline{\mathfrak{u}}$. A smooth locally defined section $s$ of $\overline{\mathfrak{u}}$ is holomorphic if and only if $D^{\prime \prime} s=0$. We'll denote this complex structure by $\left(\overline{\mathfrak{u}}, D^{\prime \prime}\right)$.

Suppose that $P \in D$ and that $t$ is a local holomorphic coordinate on $C$ centered at $P$. Since $t \Omega$ is a smooth $\overline{\mathfrak{u}}$-valued form on $C$ in a neighbourhood $\Delta$ of $P$, and since $t \nabla_{0}$ takes smooth sections of $\overline{\mathfrak{u}}$ defined on $\Delta$ to smooth 1-forms with values in $\overline{\mathfrak{u}}$, it follows that $t \nabla$ is a differential operator on sections of $\overline{\mathfrak{u}}$ over $\Delta$. This implies that $\nabla$ is a meromorphic connection on $\left(\overline{\mathfrak{u}}, D^{\prime \prime}\right)$ with regular singular points along $D$.

Proposition 7.7. The bundle $\left(\overline{\mathfrak{u}}, D^{\prime \prime}\right)$ with the connection $D^{\prime}$ is Deligne's canonical extension of $(\mathfrak{u}, \nabla)$ to $C$.

Proof. Since all singularities of $\nabla$ are regular singular points, it suffices to check that the residue of $\nabla$ at each $P \in D$ is pronilpotent endomorphism of $\mathfrak{u}_{P}$, the fiber of $\overline{\mathfrak{u}}$ over $P$. This is an immediate consequence of the fact that the residue of $\left(\operatorname{Gr}_{\mathrm{LCS}}^{\bullet} \mathfrak{u}, \nabla\right) \cong\left(\mathfrak{u}, \nabla_{0}\right)$ at $P$ is pronilpotent by assumption and that the residue of $\Omega$ at $P$ is an element of $\mathfrak{u}_{P}$, which acts trivially on $\mathrm{Gr}_{\mathrm{LCS}}^{\bullet} \mathfrak{u}_{P}$.

We now turn our attention to the behaviour of the Hodge bundles. Since each $\mathfrak{u}_{n}$ is a sum of variations of MHS that are tensor products of a constant MHS with a PVHS, they behave well near each cusp $P \in D$. In particular, the Hodge bundles $F^{p} \mathfrak{u}_{n}$ extend to sub-bundles of $\overline{\mathfrak{u}}_{n}$. This implies that the the Hodge bundles $F^{p} \mathfrak{u}$ extend to holomorphic sub-bundles of $\overline{\mathfrak{u}}$. Consequently, they extend as $C^{\infty}$ sub-bundles of $\left(\overline{\mathfrak{u}}, D^{\prime \prime}\right)$.

Lemma 7.8. The Hodge sub-bundles of $\overline{\mathbf{u}}$ are holomorphic and the connection $\nabla$ satisfies Griffiths transversality: if $s$ is a local holomorphic section of $F^{p} \overline{\mathfrak{u}}$, then $\nabla s$ is a local section $\Omega_{C}^{1}(\log D) \otimes F^{p} \overline{\mathbf{u}}$.

Proof. To prove that $F^{p} \overline{\mathbf{u}}$ is a holomorphic sub-bundle with respect to the complex structure $D^{\prime \prime}$, it suffices to show that if $s$ is a local $C^{\infty}$ section of $F^{p} \overline{\mathfrak{u}}$, then $D^{\prime \prime} s$ is a $(0,1)$-form with values in $F^{p} \overline{\mathfrak{u}}$. Since $F^{p} \mathfrak{u}$ is a holomorphic sub-bundle of $(\mathfrak{u}, \bar{\partial})$, it follows that $\bar{\partial} s$ is a $(0,1)$-form with values in $F^{p} \overline{\mathfrak{u}}$. And since

$$
\Omega^{\prime \prime} \in F^{0} K^{0,1}(C, D ; \mathfrak{u})=E^{0,1}(C) \hat{\otimes} F^{0} \overline{\mathfrak{u}}
$$

it follows that $\Omega^{\prime \prime}(s) \in E^{0,1}(C) \hat{\otimes} F^{p} \overline{\mathfrak{u}}$, which implies that $D^{\prime \prime} s \in E^{0,1}(C) \hat{\otimes} F^{p} \overline{\mathfrak{u}}$, as required.

Griffiths transversality follows for similar reasons. Suppose that $s$ is a local $C^{\infty}$ section of $F^{p} \mathfrak{u}$. Since $\left(\mathfrak{u}, \nabla_{0}\right)$ satisfies Griffiths transversality, $\nabla_{0} s$ is a 1 -form valued section of $F^{p-1} \overline{\mathfrak{u}}$. Since

$$
\Omega \in F^{0} K^{1}(C, D ; \mathfrak{u}) \subseteq E^{1}(C \log D) \hat{\otimes} F^{-1} \overline{\mathfrak{u}},
$$

$\Omega(s)$ is a 1 -form valued section of $F^{p-1} \overline{\mathfrak{u}}$. It follows that $\nabla(s)$ takes values in $F^{-1} \overline{\mathfrak{u}}$.

Lemma 7.9. The weight sub-bundles $W_{m} \mathfrak{u}$ are flat sub-bundles of $(\overline{\mathfrak{u}}, \nabla)$. Moreover, the identity induces an isomorphism of PVHS

$$
\left(\operatorname{Gr}_{m}^{W} \overline{\mathfrak{u}}, \nabla\right) \cong\left(\operatorname{Gr}_{m}^{W} \overline{\mathfrak{u}}, \nabla_{0}\right) .
$$


Proof. Both assertions follow from the fact that $\Omega$ (and hence $\Omega^{\prime \prime}$ as well) takes values in $\mathfrak{u}$ and that $\mathfrak{u}=W_{-1} \mathfrak{u}$. This implies that the adjoint action of $\Omega$ and $\Omega^{\prime \prime}$ on $\operatorname{Gr}_{\bullet}^{W} \mathfrak{u}$ is trivial. It follows that $\nabla$ respects the weight filtration of $\mathfrak{u}$ and that the induced connection on $\mathrm{Gr}_{\bullet}^{W} \mathfrak{u}$ is $\nabla_{0}$.

Since $\Omega$ acts trivially on $\mathrm{Gr}_{\mathrm{LCS}}^{\bullet} \mathfrak{u}$, we have:

Lemma 7.10. For all $n \geq 1$ there is a natural isomorphism of

$$
\left(\mathrm{Gr}_{\mathrm{LCS}}^{n} \mathfrak{u}, \nabla\right) \cong\left(\mathrm{Gr}_{\mathrm{LCS}}^{n} \mathfrak{u}, \nabla_{0}\right) \cong \mathfrak{u}_{n}
$$

local systems.

7.5. The ind-variation $\mathbb{O}\left(P_{x}\right)$. Here we dispense of a few technicalities, in preparation for the construction of a MHS on $\mathcal{O}\left(\mathcal{G}_{x}\right)$ in the next section.

Denote the fibers of $\mathbb{V}$ over $x, y \in C^{\prime}$ by $V_{x}$ and $V_{y}$, respectively. Denote the Zariski closure of the image of the parallel transport mapping

$$
\rho_{x, y}: \Pi\left(C^{\prime} ; x, y\right) \rightarrow \operatorname{Hom}\left(H_{x}, H_{y}\right)
$$

by $R_{x, y}$. Set $R_{x}=R_{x, x}$. Then $R_{x, y}$ is a left $R_{x}$ torsor and a right $R_{y}$ torsor.

The theorem of the fixed part (Sec. 7.2) implies that the coordinate ring of $R_{x, y}$ is an algebra in the category of ind-Hodge structures of weight 0 . This HS is characterized by the property that the monodromy coaction

$$
E_{x} \rightarrow \mathcal{O}\left(R_{x, y}\right) \otimes E_{y}
$$

is a morphism of HS for all polarized variations of Hodge structure $\mathbb{E}$ over $C^{\prime}$ whose monodromy representation factors through $\rho_{x}: \pi_{1}\left(C^{\prime}, x\right) \rightarrow R_{x}$. (Here $E_{z}$ denotes the fiber of $\mathbb{E}$ over $z \in C^{\prime}$.) Under our assumption that each irreducible representation $V_{\lambda}$ of $R$ is absolutely irreducible and that the corresponding local system $\mathbb{V}_{\lambda}$ underlies a PVHS over $C^{\prime}$, there is an isomorphism of HS

$$
\mathcal{O}\left(R_{x, y}\right) \cong \bigoplus_{\lambda} \operatorname{Hom}\left(V_{\lambda, x}, V_{\lambda, y}\right)^{*} .
$$

When $C^{\prime}=\Gamma \backslash \mathfrak{h}, \Gamma$ a finite index subgroup of $\mathrm{SL}_{2}(\mathbb{Z})$, and $\mathbb{H}$ is the standard variation of $\mathrm{HS}$,

$$
\mathcal{O}\left(R_{x, y}\right)=\bigoplus_{n \geq 0} \operatorname{Hom}\left(S^{n} H_{x}, S^{n} H_{y}\right)^{*} .
$$

Denote the local system over $C^{\prime}$ whose fiber over $y$ is $\mathcal{O}\left(R_{y, x}\right)$ by $\mathbb{O}_{x}$. In concrete terms

$$
\mathbb{O}_{x}=\bigoplus_{\lambda} \operatorname{Hom}\left(\mathbb{V}_{\lambda}, V_{\lambda, x}\right)^{*} \cong \bigoplus_{\lambda} \mathbb{V}_{\lambda} \otimes V_{\lambda, x}^{*} .
$$

Note that this is local system of algebras and that there is a natural left $R_{x}$ action that preserves the algebra structure.

For every local system $\mathbb{E}$ whose monodromy representation factors through $\rho$ : $\pi_{1}(X, x) \rightarrow R_{x}$, there is a natural isomorphism

$$
\mathbb{V} \cong\left[\mathbb{O}_{x} \otimes E_{x}\right]^{R}
$$

The $R$-finite vectors in the de Rham complex of $C^{\prime}$ with coefficients in $\mathbb{O}_{x}$ form a complex $E_{\text {fin }}^{\bullet}\left(C^{\prime}, \mathbb{O}_{x}\right)$. In concrete terms, this is

$$
E_{\text {fin }}^{\bullet}\left(C^{\prime}, \mathbb{O}_{x}\right)=\bigoplus_{\lambda} E^{\bullet}\left(C^{\prime}, \mathbb{V}_{\lambda}\right) \otimes V_{\lambda, x}^{*} .
$$


It is a (graded commutative) differential graded algebra. Similarly, one defines the ind-MHC

$$
\mathbf{K}\left(\mathbb{O}_{x}\right)=\bigoplus_{\lambda} \mathbf{K}\left(\mathbb{V}_{\lambda}\right) \otimes V_{\lambda, x}^{*}
$$

whose complex part is

$$
K^{\bullet}\left(C, D ; \mathbb{O}_{x}\right)=\bigoplus_{\lambda} K^{\bullet}\left(C, D ; \mathbb{V}_{\lambda}\right) \otimes V_{\lambda, x}^{*}
$$

The relevance of these complexes is that the iterated integrals of their elements are elements of the coordinate ring of $\mathcal{G}_{x}$. (Cf. [15].)

Lemma 7.13. If $\mathbb{E}$ is a PVHS over $C^{\prime}$, then the isomorphism (7.12) induces an isomorphism of bifiltered complexes:

$$
K^{\bullet}(C, D ; \mathbb{E}) \cong\left(K^{\bullet}\left(C, D ; \mathbb{O}_{x}\right) \otimes \mathbb{E}\right)^{R}
$$

The relevance of the preceding discussion is that iterated integrals of elements of

$$
E^{\bullet}\left(C^{\prime}, \mathbb{V}_{\lambda}\right) \otimes V_{\lambda, x}^{*}
$$

that occur in Section 4 are iterated integrals of elements of $E^{\mathrm{fin}}\left(C^{\prime}, \mathbb{O}_{x}\right)$ considered in [15]. This implies that the iterated Shimura integrals considered by Manin [29, 30] are examples of the iterated integrals constructed in [15].

7.6. The MHS on the relative completion of $\pi_{1}\left(C^{\prime}, x\right)$. Denote the fiber of $\mathfrak{u}$ over $x \in C^{\prime}$ by $\mathfrak{u}_{x}$. Denote the corresponding prounipotent group by $\mathcal{U}_{x}$. The Hodge and weight bundles of $\boldsymbol{u}$ restrict to Hodge and weight filtrations on $\mathfrak{u}_{x}$. Set $\Gamma=\pi_{1}\left(C^{\prime}, x\right)$ and write $C^{\prime}$ as the quotient $\Gamma \backslash X$ of a simply connected Riemann surface by $\Gamma$. Implicit here is that we have chosen a point $\tilde{x} \in X$ that lies over $x \in C^{\prime}$.

Trivialize the pullback of each local system $\mathfrak{u}_{n}$ to $X$ so that the flat sections are constant. ${ }^{12}$ This determines a trivialization

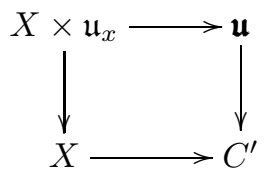

of the pullback of $\boldsymbol{u}$ to $X$ as the product of the pullbacks of the $\boldsymbol{u}_{n}$. The pullback of the connection $\nabla=\nabla_{0}+\Omega$ on $\mathfrak{u}$ is $\Gamma$-invariant with respect to the diagonal $\Gamma$-action on $X \times \mathfrak{u}_{x}$ and is of the form $d+\widetilde{\Omega}$, where

$$
\widetilde{\Omega} \in\left(E^{1}(X) \hat{\otimes} \mathfrak{u}_{x}\right)^{\Gamma} .
$$

Proposition 4.6 and the fact that $\Omega_{1}$ represents the product of the identity maps $H^{1}\left(C^{\prime}, \mathbb{V}_{\lambda}\right) \rightarrow H^{1}\left(C^{\prime}, \mathbb{V}_{\lambda}\right)$ for all $\lambda \in \check{R}$ imply that the transport of $\widetilde{\Omega}$ induces an isomorphism

$$
\Theta_{x}: \mathcal{G}_{x} \rightarrow R_{x} \ltimes \mathcal{U}_{x} .
$$

\footnotetext{
${ }^{12}$ It is also natural to trivialize $\mathfrak{u}_{1}$ (and hence all $\mathfrak{u}_{n}$ ) so that the Hodge bundles are trivialized. In this case, we are in the setup of Section 4.1. The monodromy representation will be the same as it is with this "constant trivialization". Trivializing the Hodge bundles is better when computing the MHS on completed path torsors. For example, in Section 9.1, we can trivialize $\mathcal{H}:=\mathbb{H} \otimes \mathcal{O}_{\mathfrak{h}}$ over the upper half plane $\mathfrak{h}$ using the flat sections $\mathbf{a}, \mathbf{b}$, as we do here, or we can use the sections $\mathbf{a}, \mathbf{w}$ that are adapted to the Hodge filtration. The second trivialization is better suited to studying asymptotic properties of VMHS.
} 
The MHS on $\mathcal{O}\left(\mathcal{G}_{x}\right)$ is constructed by pulling back the natural Hodge and weight filtrations on $\mathcal{O}\left(R_{x}\right) \otimes \mathcal{O}\left(\mathcal{U}_{x}\right)$, which we now recall.

To describe $\mathcal{O}\left(\mathcal{U}_{x}\right)$ we recall some basic facts about prounipotent groups. Suppose that $\mathcal{N}$ is a prounipotent group over $F$ with Lie algebra $\mathfrak{n}$. The enveloping algebra $U \mathfrak{n}$ of $\mathfrak{n}$ is a Hopf algebra. The exponential mapping exp $: \mathfrak{n} \rightarrow \mathcal{N}$ is a bijection, so we can identify $\mathcal{N}$ with the subspace $\mathfrak{n}$ of $U \mathfrak{n}$. The Poincaré-Birkhoff-Witt Theorem implies that the restriction mapping induces a Hopf algebra isomorphism

$$
\mathcal{O}(\mathcal{N}) \cong \operatorname{Hom}^{\operatorname{cts}}(U \mathfrak{n}, F) \cong \operatorname{Sym}^{\operatorname{cts}}(\mathfrak{n}):=\underset{\alpha}{\lim } \operatorname{Sym} \mathfrak{n}_{\alpha} .
$$

In particular, $\mathcal{O}\left(\mathcal{U}_{x}\right) \cong \operatorname{Hom}^{\text {cts }}\left(U \mathfrak{u}_{x}, \mathbb{C}\right)$. The construction of the Hodge and weight filtrations of $\mathfrak{u}_{x}$ and (7.11) imply that the coaction

$$
U \mathfrak{u}_{x} \rightarrow \mathcal{O}\left(R_{x}\right) \otimes U \mathfrak{u}_{x}
$$

that defines the semi direct product $R_{x} \ltimes \mathcal{U}_{x}$ preserves the Hodge and weight filtrations. ${ }^{13}$

The Hodge and weight filtrations of $\mathfrak{u}_{x}$ induce Hodge and weight filtrations on $\operatorname{Hom}^{\text {cts }}\left(U \mathfrak{u}_{x}, \mathbb{C}\right)$, and thus on $\mathcal{O}\left(\mathcal{U}_{x}\right)$. Define Hodge and weight filtrations on the coordinate ring of $R_{x} \ltimes \mathcal{U}_{x}$ via the canonical isomorphism

$$
\mathcal{O}\left(R_{x} \ltimes \mathcal{U}_{x}\right) \cong \mathcal{O}\left(R_{x}\right) \otimes \mathcal{O}\left(\mathcal{U}_{x}\right) .
$$

These pullback to Hodge and weight filtrations on $\mathcal{O}\left(\mathcal{G}_{x}\right)$ along the isomorphism $\Theta_{x}$. Equation (7.14) implies that these are compatible with the product and coproduct of $\mathcal{O}\left(R_{x} \times \mathcal{U}_{x}\right)$.

A filtration $W_{\text {. }}$ of a Lie algebra $\mathfrak{u}$ is said to be compatible with the bracket if $\left[W_{i} \mathfrak{u}, W_{j} \mathfrak{u}\right] \subseteq W_{i+j} \mathfrak{u}$ for all $i, j$. Similarly, a filtration $W$ • of a Hopf algebra $A$ is compatible with its multiplication $\mu$ and comultiplication $\Delta$ if $\mu\left(W_{i} A \otimes W_{j} A\right) \subseteq$ $W_{i+j} A$ and $\Delta\left(W_{m} A\right) \subset \sum_{i+j} W_{i} A \otimes W_{j} A$. The Hodge and weight filtrations on $\mathcal{O}\left(R_{x} \ltimes \mathcal{U}_{x}\right)$ defined above are compatible with its product and coproduct.

Denote the maximal ideal of $\mathcal{O}\left(\mathcal{G}_{x}\right)$ of functions that vanish at the identity by $\mathfrak{m}_{e}$. The Lie algebra $\mathfrak{g}_{x}$ of $\mathcal{G}_{x}$ is isomorphic to $\operatorname{Hom}\left(\mathfrak{m}_{e} / \mathfrak{m}_{e}^{2}, F\right)$ and its bracket is induced by the Lie cobracket of $\mathcal{O}\left(\mathcal{G}_{x}\right)$. The Hodge and weight filtrations of $\mathcal{O}\left(\mathcal{G}_{x}\right)$ thus induce Hodge and weight filtrations on $\mathfrak{g}_{x}$ that are compatible with its bracket.

Theorem 7.15. These Hodge and weight filtrations define a $M H S$ on $\mathcal{O}\left(\mathcal{G}_{x}\right)$ for which the multiplication and comultiplication are morphisms. This MHS agrees with the one constructed in [15].

Proof. The natural isomorphism $\Theta_{x}^{*}: \mathcal{O}\left(R_{x} \ltimes \mathcal{U}_{x}\right) \rightarrow \mathcal{O}\left(\mathcal{G}_{x}\right)$ respects $\mathbb{Q}$-structures (essentially by definition). To prove the result it suffices to show that it takes the Hodge and weight filtrations on $\mathcal{O}\left(R_{x} \ltimes \mathcal{U}_{x}\right)$ constructed above onto the Hodge and weight filtrations of the canonical MHS on $\mathcal{O}\left(\mathcal{G}_{x}\right)$ constructed in [15]. This will imply that the weight filtration defined on $\mathcal{O}\left(R_{x} \ltimes \mathcal{U}_{x}\right)$ above is defined over $\mathbb{Q}$ and that $\mathcal{O}\left(R_{x} \otimes \mathcal{U}_{x}\right)$ has a MHS and that this MHS is isomorphic to the canonical MHS on $\mathcal{O}\left(\mathcal{G}_{x}\right)$ via $\Theta_{x}^{*}$.

The first point is that Saito's MHC for computing the MHS on $H^{\bullet}\left(C^{\prime}, \mathbb{V}\right)$, which is used in [15], is a generalization of Zucker's MHC used here and agrees with it

\footnotetext{
${ }^{13}$ In fact, this is a morphism of MHS - and thus strict with respect with respect to the Hodge and weight filtrations - if we give $\mathfrak{u}_{x}$ MHS via the identification $\mathfrak{u}_{x} \cong \prod_{n}\left(\mathrm{Gr}_{n}^{\mathrm{LCS}} \mathfrak{u}\right)_{x}$. The canonical MHS on $\mathfrak{u}_{x}$ has the same underlying complex vector space, the same Hodge and weight filtrations, but its $\mathbb{Q}$-structure is deformed using the deformed connection $\nabla_{0}+\Omega$.
} 
in the curve case. The iterated integrals of elements of $K^{\bullet}(C, D ; \mathbb{V}) \otimes V_{x}^{*}$ in this paper are a special case of the iterated integrals defined in $[15, \S 5]$ by Lemma 7.13.

The next point is that, by Equation (7.2) and Lemma 7.13,

$$
\Omega \in F^{0} W_{-1} K^{1}(C, D ; \mathfrak{u}) \cong F^{0} W_{-1}\left(K^{1}\left(C, D ; \mathbb{O}_{x}\right) \hat{\otimes} \mathfrak{u}_{x}\right) .
$$

This implies, and this is the key point, that — with the Hodge and weight filtrations on the bar construction defined in $[15, \S 13]$ and the Hodge and weight filtrations on $\mathfrak{u}_{x}$ defined above - the $U \mathfrak{u}_{x}$-valued formal transport

$$
T:=1+[\widetilde{\Omega}]+[\widetilde{\Omega} \mid \widetilde{\Omega}]+[\widetilde{\Omega}|\widetilde{\Omega}| \widetilde{\Omega}]+\cdots \in B\left(\mathbb{C}, K_{\text {fin }}^{\bullet}\left(C, D ; \mathbb{O}_{x}\right), \mathbb{C}\right) \hat{\otimes} U \mathfrak{u}_{x}
$$

of $\widetilde{\Omega}$, which takes values in the completed enveloping algebra of $\mathfrak{u}_{x}$, satisfies

$$
T \in F^{0} W_{0} H^{0}\left(B\left(\mathbb{C}, K_{\text {fin }}^{\bullet}\left(C, D ; \mathbb{O}_{x}\right), \mathbb{C}\right) \hat{\otimes} U \mathfrak{u}_{x}\right) .
$$

This implies that the induced Hopf algebra homomorphism

$$
f: \mathcal{O}\left(\mathcal{U}_{x}\right) \cong \operatorname{Hom}^{\operatorname{cts}}\left(U \mathfrak{u}_{x}, \mathbb{C}\right) \rightarrow H^{0}\left(B\left(\mathbb{C}, K_{\text {fin }}^{\bullet}\left(C, D ; \mathbb{O}_{x}\right), \mathcal{O}\left(R_{x}\right)\right)\right) \cong \mathcal{O}\left(\mathcal{G}_{x}\right)
$$

that corresponds to the function

$$
\mathcal{G}_{x} \stackrel{\Theta_{x}}{\longrightarrow} R_{x} \ltimes \mathcal{U}_{x} \stackrel{\text { projn }}{\longrightarrow} \mathcal{U}_{x}
$$

preserves the Hodge and weight filtrations.

The constructions in [15] imply that the homomorphism $\pi^{*}: \mathcal{O}\left(R_{x}\right) \rightarrow \mathcal{O}\left(\mathcal{G}_{x}\right)$ induced by the projection $\pi: \mathcal{G}_{x} \rightarrow R_{x}$ is a morphism of MHS. Since the Hodge and weight filtrations of $\mathcal{O}\left(\mathcal{G}_{x}\right)$ are compatible with multiplication and since both $f$ and $\pi^{*}$ preserve the Hodge and weight filtrations filtrations, the homomorphism

$$
\mathcal{O}\left(R_{x}\right) \otimes \mathcal{O}\left(\mathcal{U}_{x}\right) \stackrel{\pi^{*} \otimes f}{\longrightarrow} \mathcal{O}\left(\mathcal{G}_{x}\right)
$$

does too. This homomorphism is $\Theta_{x}^{*}$ when $\mathcal{O}\left(R_{x}\right) \otimes \mathcal{O}\left(\mathcal{U}_{x}\right)$ is identified with $\mathcal{O}\left(R_{x} \ltimes\right.$ $\left.\mathcal{U}_{x}\right)$.

It remains to prove that this isomorphism is an isomorphism of bifiltered vector spaces. Since $\pi^{*}$ is a morphism of MHS, it suffices to show that the isomorphism $j^{*} \circ f$

$$
\mathcal{O}\left(\mathcal{U}_{x}\right) \stackrel{f}{\longrightarrow} \mathcal{O}\left(\mathcal{G}_{x}\right) \stackrel{j^{*}}{\longrightarrow} \mathcal{O}\left(\mathcal{N}_{x}\right),
$$

where $j: \mathcal{N}_{x} \rightarrow \mathcal{G}_{x}$ is the inclusion of the prounipotent radical of $\mathcal{G}_{x}$, is a bifiltered isomorphism. To prove this, it suffices to show that $\mathfrak{n}_{x} \rightarrow \mathfrak{u}_{x}$ is a bifiltered isomorphism, where $\mathfrak{n}_{x}$ denotes the Lie algebra of $\mathcal{N}_{x}$. But this follows from Lemma 7.10, which implies that $H_{1}\left(\mathfrak{u}_{x}\right)$ has a MHS and that the induced homomorphism

$$
\mathrm{Gr}_{\bullet}^{\mathrm{LCS}} \mathfrak{n}_{x} \rightarrow \mathrm{Gr}_{\bullet}^{\mathrm{LCS}} \mathfrak{u}_{x}
$$

is an isomorphism of graded MHS.

The result also gives an explicit description of the MHS on $\mathfrak{u}_{x}$.

Corollary 7.16. The Hodge and weight filtrations of the natural MHS on $\mathfrak{u}_{x}$ are those induced on it from $\mathfrak{u} ;$ its $\mathbb{Q}$-structure is the one described in Proposition 4.7.

To complete the story, we show that the $\mathfrak{u}$ is a pro-admissible variation of MHS. 
Theorem 7.17. With the Hodge and weight filtrations and $\mathbb{Q}$-structure defined above, $(\mathfrak{u}, \nabla)$ is a pro-object of the category of admissible variation of MHS over $C^{\prime}$. Its lower central series is a filtration of $\mathfrak{u}$ by pro-admissible variations of MHS. The natural isomorphism

$$
\left(\mathrm{Gr}_{\mathrm{LCS}}^{n} \mathfrak{u}, \nabla_{0}\right) \cong\left(\mathrm{Gr}_{\mathrm{LCS}}^{n} \mathfrak{u}, \nabla\right)
$$

is an isomorphism of admissible variations of MHS. In particular, there are natural MHS isomorphisms

$$
H_{1}\left(\mathfrak{u}_{x}\right) \cong \prod_{\lambda \in \check{R}} H^{1}\left(C^{\prime}, \mathbb{V}_{\lambda}\right)^{*} \otimes V_{\lambda, x}
$$

for all base points $x$ of $C^{\prime} .{ }^{14}$ Finally, for all PVHS $\mathbb{V}$ over $C^{\prime}$ whose monodromy representation factors through $\rho$, the natural homomorphism

$$
H^{\bullet}\left(\mathcal{G}_{x}, V_{x}\right)=\left[H^{\bullet}\left(\mathfrak{u}_{x}\right) \otimes V_{x}\right]^{R} \rightarrow H^{\bullet}\left(C^{\prime}, \mathbb{V}\right)
$$

is an isomorphism of MHS.

With a little more work, one can show that the local system with fiber $\mathcal{O}\left(\mathcal{G}_{x}\right)$ over $x \in C^{\prime}$ is an admissible VMHS.

Proof. Proposition 7.7 and Lemma 7.8 imply that the Hodge bundles are holomorphic sub-bundles of $\mathfrak{u}$ and extend to holomorphic sub-bundles of $\overline{\mathfrak{u}}$. Lemma 7.9 implies that the weight bundle's are flat and extend to $\overline{\mathfrak{u}}$. Theorem 7.15 implies that $\mathfrak{u}$ has a natural $\mathbb{Q}$-form and that, with respect to these structures, each fiber of $\boldsymbol{u}$ has a natural MHS.

To complete the proof, we need to show that at each $P \in D$ there is a relative weight filtration of the fiber $\mathfrak{u}_{P}$ of $\overline{\mathfrak{u}}$ over $P$. First an easily verified fact. Suppose that $\mathbb{V}$ is a PVHS over $C^{\prime}$ of weight $m$. Let $M_{\bullet}$ be the monodromy weight filtration of its fiber $V_{P}$ over $P \in D$ shifted so that it is centered at $m$. Then if $A$ is a constant MHS, then the filtration

$$
M_{r}\left(A \otimes V_{P}\right):=\sum_{i+j=r} W_{i} A \otimes M_{j} V_{P}
$$

defines a relative weight filtration of the fiber over $P \in D$ of the admissible variation of MHS $A \otimes \mathbb{V}$. From this it follows that the fiber over $P \in D$ of each

$$
H^{1}\left(C^{\prime}, \mathbb{V}_{\lambda}\right)^{*} \otimes \mathbb{V}_{\lambda}
$$

has a relative weight filtration. Adding these implies that the fiber over $P \in D$ of each of the pro-variations $\mathfrak{u}_{n}$ has a relative weight filtration. Write the residue at $P \in D$ of $\nabla$ as the sum

$$
N_{P}=N_{0}+N_{\mathfrak{u}}
$$

of the residues of $\nabla_{0}$ and $\Omega$. The discussion above implies that the product of the weight filtrations on the fibers over $P$ of the $\mathfrak{u}_{n}$ defines a relative weight filtration for $N_{0}$ on the fiber $\mathfrak{u}_{P}$ of $\overline{\mathfrak{u}}$ over $P$. We have to show that this is also a relative weight filtration for $N$. To prove this, it suffices to show that $N_{\mathfrak{u}} \in W_{-2} \mathfrak{u}_{P}$. (See the definition of the relative weight filtration in [41].)

Let $t$ be a local holomorphic coordinate on $C$ centered at $P$. Then, near $P$, we can write

$$
\Omega=N_{\mathfrak{u}} \otimes \frac{d t}{t}+\text { a smooth } 1 \text {-form with values in } \overline{\mathfrak{u}}
$$

\footnotetext{
${ }^{14}$ This statement holds, even when $x$ is a tangential base point.
} 
Since $\Omega^{1} \in W_{-1} K^{1}(C, D ; \mathfrak{u})$, and since $d t / t$ has weight 1 , we see that $N_{\mathfrak{u}} \in W_{-2} \mathfrak{u}_{P}$, as required.

The last statement is a direct consequence of (3.4.2), the de Rham construction of the homomorphism in Section 4.2, and the fact that $\Omega \in F^{0} W_{-1} K^{1}(C, D ; \mathfrak{u})$, which implies that $\theta_{\Omega}: H^{0}\left(C^{\prime}, \operatorname{Hom}(\Lambda \bullet \mathfrak{u}, \mathbb{V})\right) \rightarrow K^{\bullet}(C, D, \mathbb{V})$ preserves the Hodge filtration and satisfies

$$
\theta_{\Omega}\left(W_{m} H^{0}\left(C^{\prime}, \operatorname{Hom}\left(\Lambda^{j} \mathfrak{u}, \mathbb{V}\right)\right)\right) \subseteq W_{m-j} K^{j}(C, D, \mathbb{V})
$$

in degree $j .{ }^{15}$ The last ingredient is the Theorem of the Fixed Part, which implies that for each $x \in C^{\prime}$, the restriction mapping

$$
H^{0}\left(C^{\prime}, \operatorname{Hom}\left(\Lambda^{\bullet} \mathfrak{u}, \mathbb{V}\right)\right) \rightarrow \mathcal{C}^{\bullet}\left(\mathfrak{u}_{x}, V_{x}\right)^{R}
$$

is an isomorphism of MHS for all $x \in C^{\prime}$.

7.7. A MHS on completed path torsors. Here we give a brief description of how to extend the methods of the previous section to construct the canonical MHS on the coordinate ring $\mathcal{O}\left(\mathcal{G}_{x, y}\right)$ of the relative completion of the path torsor $\Pi\left(C^{\prime} ; x, y\right)$ of $C^{\prime}$ with respect to a polarized VHS $\mathbb{V}$. Here $C^{\prime}$ may be an orbifold of the form $\Gamma \backslash X$.

As in Section 7.6, write $C^{\prime}$ as the quotient of a simply connected Riemann surface $X$ by a discrete group $\Gamma$ isomorphic to $\pi_{1}\left(C^{\prime}, x\right)$. Trivialize the pullback of each $\mathfrak{u}_{n}$ to $X$ using the flat sections and use this to trivialize the pullback of $\mathfrak{u}$ to $X$. Here, unlike in the previous section, it is useful to denote the common fiber of the trivialization by $\mathfrak{u}$ so that the pullback of $\mathfrak{u}$ to $X$ is $X \times \mathfrak{u}$. The fiber over $t \in X$ will be regarded as $\mathfrak{u}$ with a Hodge filtration $F_{t}^{\bullet}$, which depends on $t$, and a weight filtration $W_{\bullet}$, which does not. We can therefore identify $\operatorname{Hom}^{\text {cts }}\left(\mathfrak{u}_{s}, \mathfrak{u}_{t}\right)$ with End $^{\text {cts }} \mathfrak{u}$. The Hodge and weight filtrations of $\mathfrak{u}_{s}$ and $\mathfrak{u}_{t}$ induce Hodge and weight filtrations on $\operatorname{Hom}^{\text {cts }}\left(\mathfrak{u}_{s}, \mathfrak{u}_{t}\right)$.

For $s, t \in X$, set $\mathcal{U}_{s, t}$ be the subscheme of $\operatorname{Isom}^{\text {cts }}\left(\mathfrak{u}_{s}, \mathfrak{u}_{t}\right)$ that corresponds to the subgroup $\mathcal{U}:=\exp \mathfrak{u}$ of Aut $\mathfrak{u}$. It is a proalgebraic variety. Denote the subspace of $\operatorname{Hom}^{\text {cts }}\left(\mathfrak{u}_{s}, \mathfrak{u}_{t}\right)$ that corresponds to the image of

$$
U \mathfrak{u} \in \operatorname{End}(\mathfrak{u}), \quad u \mapsto\{v \mapsto u v\}
$$

by $U \mathfrak{u}_{s, t}$. The coordinate ring of $\mathcal{U}_{s, t}$ is $\mathcal{O}\left(\mathcal{U}_{s, t}\right) \cong \operatorname{Hom}^{\operatorname{cts}}\left(U \mathfrak{u}_{s, t}, \mathbb{C}\right)$. It has natural Hodge and weight filtrations induced from those on $\operatorname{Hom}^{\mathrm{cts}}\left(\mathfrak{u}_{s}, \mathfrak{u}_{t}\right)$.

The pullback connection is $d+\widetilde{\Omega}$, where $\widetilde{\Omega} \in E^{1}(X) \hat{\otimes} \mathfrak{u}$. Since the structure group of this connection is $\mathcal{U}$, the parallel transport map $T_{s, t}: \mathfrak{u}_{s} \rightarrow \mathfrak{u}_{t}$ lies in $\mathcal{U}_{s, t}$.

As in Section 5, we choose a fundamental domain $D$ of the action of $\Gamma$ on $X$. Denote the unique lift of $z \in C^{\prime}$ to $D$ by $\tilde{z}$. For each homotopy class of paths in $C^{\prime}$ from $x$ to $y$ one has $\gamma \in \Gamma$ and a homotopy class $c_{\gamma}$ of paths from $\tilde{x}$ to $\gamma \tilde{y}$. Parallel transport defines a function

$$
\Theta_{x, y}: \Pi\left(C^{\prime} ; x, y\right) \rightarrow \mathcal{U}_{x, y} \times R_{x, y} \cong \mathcal{G}_{x, y}
$$

by $\left(\gamma, c_{\gamma}\right) \mapsto\left(T\left(c_{\gamma}\right)^{-1}, \rho_{x, y}(\gamma)\right)$. This is the relative completion of $\Pi\left(C^{\prime} ; x, y\right)$.

The isomorphism

$$
\mathcal{O}\left(\mathcal{G}_{x, y}\right) \cong \mathcal{O}\left(\mathcal{U}_{x, y}\right) \otimes \mathcal{O}\left(R_{x, y}\right)
$$

\footnotetext{
${ }^{15}$ This is equivalent to the statement that $\theta_{\Omega}$ is a filtration preserving dga homomorphism to $\operatorname{Dec}_{W} K^{j}(C, D, \mathbb{V})$, where $\operatorname{Dec}_{W}$ is Deligne's shifting functor (with respect to the weight filtration), [11].
} 
induces Hodge and weight filtrations on the coordinate ring of $\mathcal{G}_{x, y}$. It also has a natural $\mathbb{Q}$-structure as relative completion is defined over $\mathbb{Q}$ and behaves well under base change from $\mathbb{Q}$ to $\mathbb{C}$.

The following theorem generalizes Theorems 7.15 and 7.17. It is proved using a similar arguments. A more general version of all but the last statement is proved in $[15, \S 12-13]$.

Theorem 7.18. These Hodge and weight filtrations define a MHS on $\mathcal{O}\left(\mathcal{G}_{x, y}\right)$, making it a ring in the category of ind-mixed Hodge structures. This MHS coincides with the one constructed in [15]. It has the property that if $x, y, z \in X$, then the maps $\mathcal{G}_{x, y} \rightarrow \mathcal{G}_{y, x}$ and $\mathcal{G}_{x, y} \times \mathcal{G}_{y, z} \rightarrow \mathcal{G}_{x, z}$ induced by inverse and path multiplication, respectively, induce morphisms of $M H S$

$$
\mathcal{O}\left(\mathcal{G}_{y, x}\right) \rightarrow \mathcal{O}\left(\mathcal{G}_{x, y}\right) \text { and } \mathcal{O}\left(\mathcal{G}_{x, z}\right) \rightarrow \mathcal{O}\left(\mathcal{G}_{x, y}\right) \otimes \mathcal{O}\left(\mathcal{G}_{y, z}\right) .
$$

In addition the local system $\mathcal{G}_{x}$, over $C^{\prime}$ whose fiber over $x, y$ is $\mathcal{O}\left(\mathcal{G}_{x, y}\right)$ is an Ind object of the category of admissible variations of $M H S$ over $C^{\prime}$.

7.8. Tangential base points and limit MHSs. Theorem 7.17 implies that for each choice of a non-zero tangent vector $\vec{v}$ of $C$ at $P \in D$, there is a limit MHS on the fiber $\mathfrak{u}_{P}$ of $\overline{\mathfrak{u}}$ at $P$. We will denote this MHS by $\mathfrak{u}_{\vec{v}}$. It is natural to think of it as a MHS on the unipotent radical of the relative completion of the fundamental group $\pi_{1}\left(C^{\prime}, \vec{v}\right)$ of $C^{\prime}$ with (tangential) base point $\vec{v}$.

More generally, we consider path torsors between tangential base points,. We first recall the definition from [12] of the torsor of paths $\Pi\left(C^{\prime} ; \vec{v}, \vec{w}\right)$. Here $P, Q \in D$ and $\vec{v} \in T_{P} C, \vec{w} \in T_{Q} C$ are non-zero tangent vectors. Elements of $\Pi(C ; \vec{v}, \vec{w})$ are homotopy classes of piecewise smooth paths $\gamma: I \rightarrow C$ satisfying

(i) $\gamma(0)=P, \gamma(1)=Q$,

(ii) $\gamma(t) \in C^{\prime}$, when $0<t<1$,

(iii) $\gamma^{\prime}(0)=\vec{v}$ and $\gamma^{\prime}(1)=-\vec{w}$.

This definition can be modified to define $\Pi(C ; \vec{v}, x)$ and $\Pi(C ; x, \vec{w})$ when $x \in C^{\prime}$. One defines $\pi_{1}\left(C^{\prime}, \vec{v}\right)=\Pi\left(C^{\prime}, \vec{v}, \vec{v}\right)$. For any 3 base points (tangential or regular)

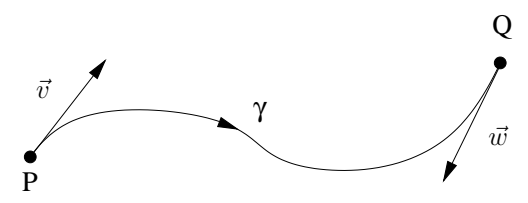

Figure 2. A path from $\vec{v}$ to $\vec{w}$ in $C^{\prime}$

$b, b^{\prime}, b^{\prime \prime}$, there are composition maps

$$
\Pi\left(C^{\prime} ; b, b^{\prime}\right) \times \Pi\left(C^{\prime} ; b^{\prime}, b^{\prime \prime}\right) \rightarrow \Pi\left(C^{\prime} ; b, b^{\prime \prime}\right) .
$$

7.8.1. The MHS on $\mathfrak{u}_{\vec{v}}$. Suppose that $P \in D$ and that $\vec{v}$ is a non-zero tangent vector of $C$ at $P$. The complex vector space underlying the limit MHS on $\mathfrak{u}_{\vec{v}}$ is the fiber $\mathfrak{u}_{P}$ of $\overline{\mathfrak{u}}$ over $P$. Its Hodge and weight filtrations $F^{\bullet}$ and $W \bullet$ are the restrictions to $\mathfrak{u}_{P}$ of the Hodge and weight filtrations of $\overline{\mathfrak{u}}$ that were constructed above. There is also the relative weight filtration $M_{\bullet}$ of $\mathfrak{u}_{P}$, which was constructed in the proof of Theorem 7.17. These data depend only on $P$ and not on $\vec{v}$. 
To construct the $\mathbb{Q}$-structure, choose a local holomorphic coordinate $t: \Delta \rightarrow \mathbb{C}$ on $C$ centered at $P$ with the property that $\vec{v}=\partial / \partial t$. Then there is a unique trivialization $\left.\overline{\mathfrak{u}}\right|_{\Delta} \cong \Delta \times \mathfrak{u}_{P}$ of $\overline{\mathfrak{u}}$ over $\Delta$ such that (1) the trivialization is the identity on the fiber $\mathfrak{u}_{P}$ over $P$, (2) $\Delta \cap D=\{P\}$, and (3) $\nabla=d+N_{P} d t / t$, where $N_{P}$ is the residue of $\nabla$ at $P$. This trivialization allows us to identify fibers of $\mathfrak{u}$ over points near $P$ with $\mathfrak{u}_{P}$. Note that this identification depends on the choice of the local coordinate $t$.

For $t \in \Delta$, the $\mathbb{Q}$-structure on $\mathfrak{u}_{P}$ corresponding to $t \vec{v}$ is simply the $\mathbb{Q}$ structure on $\mathfrak{u}_{P}$ obtained by identifying $\mathfrak{u}_{P}$ with the fiber $\mathfrak{u}_{t}$ of $\mathfrak{u}$ over $t \in \Delta$ and taking the $\mathbb{Q}$-structure to be that of $\mathfrak{u}_{t}$. The $\mathbb{Q}$-structure corresponding to $\vec{v}$ is

$$
\mathfrak{u}_{\vec{v}, \mathbb{Q}}:=t^{N_{P}} \mathfrak{u}_{t \vec{v}, \mathbb{Q}} \cdot
$$

That is, it is the unique $\mathbb{Q}$-structure on $\mathfrak{u}_{P}$ such that

$$
u_{t \vec{v}, \mathbb{Q}}:=t^{-N_{P}} \mathfrak{u}_{\vec{v}, \mathbb{Q}}
$$

for all $t \in \mathbb{C}^{*}$. Although the trivialization above depends on the choice of the local coordinate $t$, the $\mathbb{Q}$-structure on $\mathfrak{u}_{P}$ corresponding to $\vec{v}$ depends only on $\vec{v}$ the tangent vector $\vec{v}$.

With a little more effort, one can construct the limit MHS on $\mathcal{O}\left(\mathcal{G}_{\vec{v}}\right)$. Full details will appear in [24]. As in the case of cohomology, where periods of limit MHSs can be computed by regularizing integrals, the periods of the limit MHS on $\mathfrak{u}_{\vec{v}}$ are regularized iterated integrals.

7.8.2. Limit $M H S$ on completed path torsors. Similarly, one can construct the limit MHS on $\mathcal{O}\left(\mathcal{G}_{x, \vec{w}}\right)$ and $\mathcal{O}\left(\mathcal{G}_{\vec{v}}, \vec{w}\right)$, etc. Full details will appear in [24].

\section{Completed Path Torsors and Admissible Variations of MHS}

Here we state two results that relate the Hodge theory of relative completion of fundamental groups and path torsors to admissible variations of MHS. These are special cases of results in [24].

Let $C, D$ and $C^{\prime}=C-D$ be as above. Let $\mathbb{V}$ be a PVHS over $C^{\prime}$ and $R_{x}$ the Zariski closure of the monodromy representation $\pi_{1}\left(C^{\prime}, x\right) \rightarrow$ Aut $V_{x}$. Let $\rho_{x}$ be the homomorphism $\pi_{1}(X, x) \rightarrow R_{x}$. Let $\mathcal{G}_{x}$ be the completion of $\pi_{1}\left(C^{\prime}, x\right)$ with respect to $\rho_{x}$. For base points (regular or tangential) $b, b^{\prime}$ of $C^{\prime}$, let $\mathcal{G}_{x, y}$ denote the relative completion of $\Pi\left(C^{\prime} ; b, b^{\prime}\right)$.

Denote by $\operatorname{MHS}\left(C^{\prime}, \mathbb{V}\right)$ the category of admissible VMHS $\mathbb{A}$ over $C^{\prime}$ with the property that the monodromy representation of $\mathrm{Gr}_{\bullet}^{W} \mathbb{A}$ factors through $\rho_{x}$. This condition implies that the monodromy representation

$$
\pi_{1}\left(C^{\prime}, x\right) \rightarrow \operatorname{Aut}\left(A_{x}\right)
$$

factors through $\pi_{1}\left(C^{\prime}, x\right) \rightarrow \mathcal{G}_{x}$.

Theorem 8.1. For all variations $\mathbb{A}$ in $\mathrm{MHS}\left(C^{\prime}, \mathbb{V}\right.$ ) and all base points $b, b^{\prime}$ (possibly tangential) of $C^{\prime}$ the parallel transport mapping

$$
A_{b} \rightarrow A_{b^{\prime}} \otimes \mathcal{O}\left(\mathcal{G}\left(\mathcal{G}_{b, b^{\prime}}\right)\right)
$$

induced by $A_{b} \times \Pi\left(C^{\prime} ; b, b^{\prime}\right) \rightarrow A_{b^{\prime}}$ is a morphism of MHS. When $b$ or $b^{\prime}$ is tangential, then the monodromy preserves both the weight filtration $W_{\bullet}$ and the relative weight filtration $M_{\bullet}$. 
Definition 8.2. Suppose that $\mathcal{G}$ is a proalgebraic group whose coordinate ring $\mathcal{O}(\mathcal{G})$ is a Hopf algebra in the category of ind mixed Hodge structures. A Hodge representation of $\mathcal{G}$ on a MHS $A$ is a homomorphism for which the action

$$
A \rightarrow A \otimes \mathcal{O}(\mathcal{G})
$$

is a morphism of MHS. The category of Hodge representations of $\mathcal{G}$ will be denoted by $\operatorname{HRep}(\mathcal{G})$.

The previous result implies that taking the fiber at $b$ defines a functor from $\operatorname{MHS}\left(C^{\prime}, \mathbb{V}\right)$ to $\operatorname{HRep}\left(\mathcal{G}_{b}\right)$. The next theorem follows from Theorem 7.15 by a tannakian argument. Full details will be given in [24].

Theorem 8.3. For all base points $b$ of $C^{\prime}$, the "fiber at $b$ " functor $\operatorname{MHS}\left(C^{\prime}, \mathbb{V}\right) \rightarrow$ $\operatorname{HRep}\left(\mathcal{G}_{b}\right)$ is an equivalence of categories.

\section{Part 2. Completed Path Torsors of Modular Curves}

In this part, we apply the general constructions of the first part to explore the relative completions of modular groups, mainly in the case of the full modular group $\mathrm{SL}_{2}(\mathbb{Z})$. Throughout we use the following notation.

Suppose that $\Gamma$ is a finite index subgroup of $\mathrm{SL}_{2}(\mathbb{Z})$. The associated curve $X_{\Gamma}$ is the quotient $\Gamma \backslash \mathfrak{h}$ of the upper half plane by $\Gamma$. It is a smooth affine curve when $\Gamma$ is torsion free. When $\Gamma$ has torsion, it will be regarded as an orbifold as follows: Choose a finite index, torsion free normal subgroup $\Gamma^{\prime}$ of $\Gamma$. Set $G=\Gamma / \Gamma^{\prime}$. Then $X_{\Gamma}$ is the orbifold quotient of $X_{\Gamma^{\prime}}$ by $G$. To work on the orbifold $X_{\Gamma}$, one can work either $G$-equivariantly on $X_{\Gamma^{\prime}}$ or $\Gamma$-equivariantly on $\mathfrak{h}$.

The (orbi) curve $X_{\Gamma}$ can be completed to a smooth (orbi) curve by adding the finite set $D:=\Gamma \backslash \mathbb{P}^{1}(\mathbb{Q})$ of "cusps". Denote the compactified curve by $\bar{X}_{\Gamma}$. When $\Gamma=\mathrm{SL}_{2}(\mathbb{Z})$, the modular curve $X_{\Gamma}$ is the moduli space $\mathcal{M}_{1,1}$ of elliptic curves and $\bar{X}_{\Gamma}$ is $\overline{\mathcal{M}}_{1,1}$, its the Deligne-Mumford compactification, which is obtained by adding a single cusp.

If $P \in D$ is in the orbit of $\infty \in \mathbb{P}^{1}(\mathbb{Q})$, then

$$
\Gamma \cap\left(\begin{array}{cc}
1 & \mathbb{Z} \\
0 & 1
\end{array}\right)=\left(\begin{array}{cc}
1 & n \mathbb{Z} \\
0 & 1
\end{array}\right) .
$$

for some $n \geq 1$. A punctured neighbourhood of $P$ in $X_{\Gamma}$ is the quotient of $\{\tau \in$ $\mathbb{C}: \operatorname{Im}(\tau)>1\}$ by this group. This is a punctured disk with coordinate $e^{2 \pi i n \tau}$. In particular, when $\Gamma=\mathrm{SL}_{2}(\mathbb{Z})$, the coordinate about the cusp is $q:=e^{2 \pi i \tau}$.

\section{The Variation of Hodge Structure $\mathbb{H}$}

9.1. The Local System $\mathbb{H}$. The universal elliptic curve $f: \mathcal{E} \rightarrow X_{\Gamma}$ over $X_{\Gamma}$ is the quotient of $\mathbb{C} \times \mathfrak{h}$ by $\Gamma \ltimes \mathbb{Z}^{2}$, which acts via

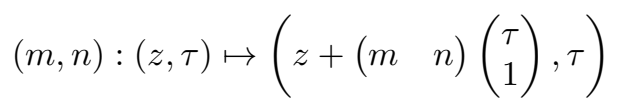

and $\gamma:(z, \tau) \mapsto\left((c \tau+d)^{-1} z, \gamma \tau\right)$, where

$$
\gamma=\left(\begin{array}{ll}
a & b \\
c & d
\end{array}\right) \in \Gamma .
$$

When $\Gamma$ is not torsion free, it should be regarded as an orbifold family of elliptic curves. 
The local system $\mathbb{H}$ over $X_{\Gamma}$ is $R^{1} f_{*} \mathbb{Q}$. When $\Gamma$ is torsion free, this is the local system over $X_{\Gamma}$ with fiber $H^{1}\left(f^{-1}(x), \mathbb{Q}\right)$ over $x$. It is a polarized variation of HS of weight 1 . Since Poincaré duality induces an isomorphism

$$
H_{1}(E) \cong H^{1}(E)(1)
$$

for all elliptic curves $E$, the polarized variation $\mathbb{H}(1)$ of weight -1 is the local system over $X_{\Gamma}$ whose fiber over $x$ is $H_{1}\left(f^{-1}(x), \mathbb{Q}\right)$. The polarization is the intersection pairing. Denote the corresponding holomorphic vector bundle $\mathbb{H} \otimes \mathcal{O}_{X_{\Gamma}}$ by $\mathcal{H}$. Its Hodge filtration satisfies

$$
\mathcal{H}=F^{0} \mathcal{H} \supset F^{1} \mathcal{H} \supset F^{2} \mathcal{H}=0 .
$$

The only interesting part is $F^{1} \mathcal{H}$.

In general we will work with the pullback $\mathbb{H}_{\mathfrak{h}}$ of $\mathbb{H}$ to $\mathfrak{h}$. Its fiber over $\tau \in \mathfrak{h}$ is $H^{1}\left(E_{\tau}, \mathbb{Z}\right)$, where

$$
E_{\tau}:=\mathbb{C} / \Lambda_{\tau} \text { and } \Lambda_{\tau}:=\mathbb{Z} \oplus \mathbb{Z} \tau .
$$

Denote the basis of $H_{1}\left(E_{\tau}, \mathbb{Z}\right)$ corresponding to the elements 1 and $\tau$ of $\Lambda_{\tau}$ by $\mathbf{a}, \mathbf{b}$.

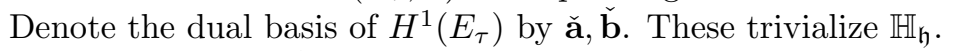

If we identify $H^{1}\left(E_{\tau}\right)$ with $H_{1}\left(E_{\tau}\right)$ via Poincaré duality, then

$$
\check{\mathbf{a}}=-\mathbf{b} \text { and } \check{\mathbf{b}}=\mathbf{a} \text {. }
$$

We regard these as sections of $\mathbb{H}_{\mathfrak{h}}$.

For each $\tau \in \mathfrak{h}$, let $\omega_{\tau} \in H^{0}\left(E_{\tau}, \Omega^{1}\right)$ be the unique holomorphic differential that takes the value 1 on a. It spans $F^{1} H^{1}\left(E_{\tau}\right)$. In terms of the framing, it is given by

$$
\omega_{\tau}=\check{\mathbf{a}}+\tau \check{\mathbf{b}}=\tau \mathbf{a}-\mathbf{b}=\left(\begin{array}{ll}
\mathbf{a} & -\mathbf{b}
\end{array}\right)\left(\begin{array}{l}
\tau \\
1
\end{array}\right) .
$$

The map $\omega: \tau \mapsto \omega_{\tau}$ is thus a holomorphic section of $\mathcal{H}_{\mathfrak{h}}:=\mathbb{H}_{\mathfrak{h}} \otimes \mathcal{O}_{\mathfrak{h}}$ whose image spans $F^{1} \mathcal{H}_{\mathfrak{h}}$.

Since $\mathfrak{h}$ is contractible, $H_{1}(\mathcal{E}, \mathbb{Z}) \cong \mathbb{Z} \mathbf{a} \oplus \mathbb{Z} \mathbf{b}$. The left action of $\mathrm{SL}_{2}(\mathbb{Z})$ on $\mathcal{E}$ induces a left action on $H_{1}(\mathcal{E})$, and therefore a right action on frames. The following result gives a formula for this action on frames.

Lemma 9.2. For all $\gamma \in \Gamma$,

(i) $\gamma:\left(\begin{array}{ll}\mathbf{a} & -\mathbf{b}\end{array}\right) \mapsto(\mathbf{a}-\mathbf{b}) \gamma$,

(ii) the section $\omega$ of $\mathcal{H}_{\mathfrak{h}}$ satisfies $(1 \otimes \gamma) \omega=(c \tau+d)\left(\gamma^{*} \otimes 1\right) \omega$.

Proof. Let $\gamma=\left(\begin{array}{ll}a & b \\ c & d\end{array}\right)$. Regard $\mathbf{a}$ and $\mathbf{b}$ as sections of $\mathbb{H}_{\mathfrak{h}}$. Denote the values of $\mathbf{a}$ and $\mathbf{b}$ at $\tau$ by $\mathbf{a}, \mathbf{b}$ and at $\gamma(\tau)$ by $\mathbf{a}^{\prime}, \mathbf{b}^{\prime}$. Then (cf. Figure 3 )

$$
\left(\begin{array}{ll}
\mathbf{a}^{\prime} & -\mathbf{b}^{\prime}
\end{array}\right)=\left(\begin{array}{ll}
\mathbf{a} & -\mathbf{b}
\end{array}\right)\left(\begin{array}{cc}
d & -b \\
-c & a
\end{array}\right)=\left(\begin{array}{ll}
\mathbf{a} & -\mathbf{b}
\end{array}\right) \gamma^{-1}
$$

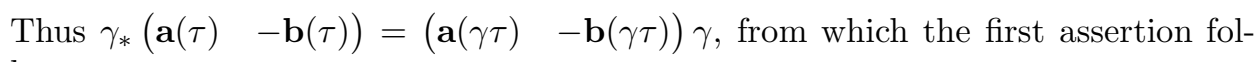
lows.

The second assertion now follows:

$$
(1 \otimes \gamma) \omega=\left(\begin{array}{ll}
\mathbf{a} & -\mathbf{b}
\end{array}\right) \gamma\left(\begin{array}{l}
\tau \\
1
\end{array}\right)=(c \tau+d)\left(\begin{array}{ll}
\mathbf{a} & -\mathbf{b}
\end{array}\right)\left(\begin{array}{c}
\gamma \tau \\
1
\end{array}\right)=(c \tau+d)\left(\gamma^{*} \otimes 1\right) \omega
$$




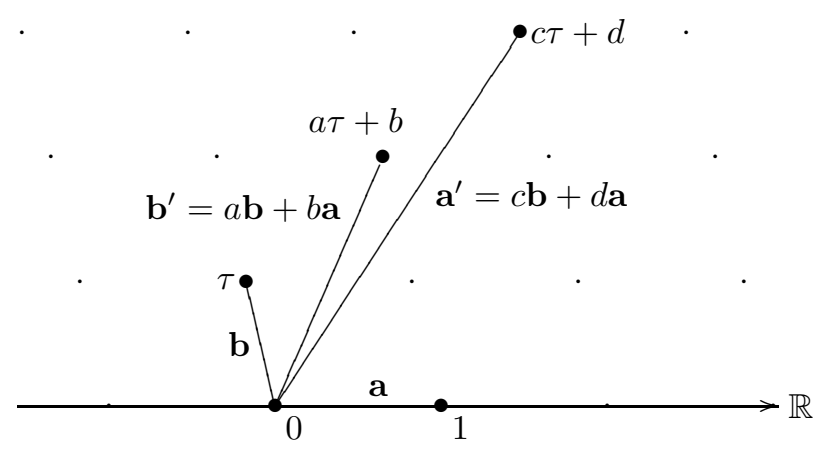

Figure 3. The $\mathrm{SL}_{2}(\mathbb{Z})$ action on frames

Define $\mathbf{w}$ to be the section

$$
\mathbf{w}: \tau \mapsto 2 \pi i \omega_{\tau}=2 \pi i(\tau \mathbf{a}-\mathbf{b}) .
$$

of $\mathcal{H}_{\mathfrak{h}}$. Since $\langle\mathbf{w}, \mathbf{a}\rangle=2 \pi i\langle\tau \mathbf{a}-\mathbf{b}, \mathbf{a}\rangle=2 \pi i$. $\mathbf{w}, \mathbf{a}$ is a framing of $\mathcal{H}_{\mathfrak{h}}$. The sections $\mathbf{a}$ and $\mathbf{w}$ trivialize $\mathcal{H}_{\mathfrak{h}}$ over $\mathfrak{h}$. As we shall see below, this trivialization is better suited to computing limit MHSs. The following computation is immediate. The proof of an equivalent formula can be found in [20, Ex. 3.4].

Corollary 9.4. The factor of automorphy associated to the trivialization

$$
\mathcal{H}_{\mathfrak{h}} \cong(\mathbb{C a} \oplus \mathbb{C w}) \times \mathfrak{h}
$$

of the pullback of $\mathcal{H}$ to $\mathfrak{h}$ is

$$
M_{\gamma}(\tau):\left(\begin{array}{ll}
\mathbf{a} & \mathbf{w}
\end{array}\right)=\left(\begin{array}{ll}
\mathbf{a} & \mathbf{w}
\end{array}\right)\left(\begin{array}{cc}
(c \tau+d)^{-1} & 0 \\
c / 2 \pi i & c \tau+d
\end{array}\right) .
$$

That is, the action of $\mathrm{SL}_{2}(\mathbb{Z})$ on $(\mathbb{C a} \oplus \mathbb{C w}) \times \mathfrak{h}$ is

$$
\gamma:(\mathbf{a}, \tau) \mapsto\left((c \tau+d)^{-1} \mathbf{a}+(c / 2 \pi i) \mathbf{w}, \gamma \tau\right) \text { and } \gamma:(\mathbf{w}, \tau) \mapsto((c \tau+d) \mathbf{w}, \gamma \tau) \text {. }
$$

The sections $\mathbf{a}$ and $\mathbf{w}$ of $\mathcal{H}_{\mathfrak{h}}$ are both invariant under $\tau \mapsto \tau+1$. They therefore descend to sections of the quotient $\mathcal{H}_{\mathbb{D}^{*}} \rightarrow \mathbb{D}^{*}$ of $\mathcal{H}_{\mathfrak{h}} \rightarrow \mathfrak{h}$ by $\left(\begin{array}{cc}1 & \mathbb{Z} \\ 0 & 1\end{array}\right)$. They trivialize $\mathcal{H}_{\mathbb{D}^{*}}$. The vector bundle

$$
\overline{\mathcal{H}}_{\mathbb{D}}:=\mathcal{O}_{\mathbb{D}} \mathbf{a} \oplus \mathcal{O}_{\mathbb{D}} \mathbf{w}
$$

is thus an extension of $\mathcal{H}_{\mathbb{D} *}$ to the $q$-disk. The Hodge bundle $F^{1} \mathcal{H}_{\mathbb{D}^{*}}$ extends to the sub-bundle $\mathcal{O}_{\mathbb{D}} \mathbf{w}$ of $\overline{\mathcal{H}}_{\mathbb{D}}$.

Denote the natural flat connection on $\mathcal{H}$ by $\nabla_{0}$. Equation (9.1) implies that

$$
\nabla_{0}\left(\begin{array}{ll}
\mathbf{a} & \mathbf{w}
\end{array}\right)=\left(\begin{array}{ll}
\mathbf{a} & \mathbf{w}
\end{array}\right)\left(\begin{array}{cc}
0 & 2 \pi i \\
0 & 0
\end{array}\right) d \tau=\left(\begin{array}{ll}
\mathbf{a} & \mathbf{w}
\end{array}\right)\left(\begin{array}{ll}
0 & 1 \\
0 & 0
\end{array}\right) \frac{d q}{q}
$$

from which it follows that the connection on $\overline{\mathcal{H}}_{\mathbb{D}}$ is

$$
\nabla_{0}=d+\mathbf{a} \frac{\partial}{\partial \mathbf{w}} \otimes \frac{d q}{q} .
$$

Since this connection is meromorphic with a simple pole at $q=0$ and nilpotent residue, the bundle $\overline{\mathcal{H}}_{\mathbb{D}}$ is the canonical extension of $\mathcal{H}_{\mathbb{D}^{*}}$ to $\mathbb{D}$. It is the pullback 
to the $q$-disk of the canonical extension of $\overline{\mathcal{H}} \rightarrow \overline{\mathcal{M}}_{1,1}$ of $\mathcal{H} \rightarrow \mathcal{M}_{1,1}$. Denote the fiber of $\overline{\mathcal{H}}$ over $q=0$ by $H$. It has basis $\mathbf{a}$, w, where $\mathbf{w}$ spans $F^{1} H$.

We now compute the limit MHSs $H_{\vec{v}}$ on $\mathbb{H}$ at $q=0$ associated to the non-zero tangent vector $\vec{v}$ of $q=0$. It will have integral lattice $H_{\mathbb{Z}}$, complexification $H$ and $F^{0} H$ defined by

$$
H_{\mathbb{Z}}=\mathbb{Z} \mathbf{a} \oplus \mathbb{Z} \mathbf{b}, H=\mathbb{C} \mathbf{a} \oplus \mathbb{C w} \text { and } F^{0} H=\mathbb{C} \mathbf{w} \subset H .
$$

To specify the MHS, we give an isomorphism $\left(H_{\mathbb{Z}}\right) \otimes \mathbb{C} \cong H$, which will depend on the tangent vector $\vec{v}^{16}$

Proposition 9.6. The $\mathbb{Z}-M H S H_{\vec{v}}(1)$ on $H$ that corresponds to the non-zero tangent vector $\vec{v}=z \partial / \partial q$ is the $M H S$ determined by the linear isomorphism $\left(H_{\mathbb{Z}}\right) \otimes \mathbb{C} \cong$ $H_{\mathbb{C}}$ given by

$$
\left(\begin{array}{ll}
\mathbf{a} & \mathbf{w}
\end{array}\right)=\left(\begin{array}{ll}
\mathbf{a} & -\mathbf{b}
\end{array}\right)\left(\begin{array}{cc}
1 & \log z \\
0 & 2 \pi i
\end{array}\right) .
$$

It is the extension of $\mathbb{Z}(-1)$ by $\mathbb{Z}(0)$ that corresponds to $z \in \mathbb{C}^{*}$ under the standard isomorphism $\operatorname{Ext}_{\mathrm{MHS}}^{1}(\mathbb{Z}(-1), \mathbb{Z}(0)) \cong \mathbb{C}^{*}$. It splits if and only if $\vec{v}=\partial / \partial q$.

Proof. Most of this is proved above. The integral lattice of $H_{z \partial / \partial q}$ is computed using the standard prescription and the fact that the value of $\mathbf{w}$ at $z \in \mathbb{D}^{*}$ is

$$
\mathbf{w}(z)=\log z \mathbf{a}+2 \pi i(-\mathbf{b}),
$$

which follows from (9.1). The weight filtration $M_{\bullet}$ of the limit MHS is the monodromy filtration of the nilpotent endomorphism $\mathbf{a} \partial / \partial \mathbf{w}$ shifted by the weight 1 of $H$ :

$$
M_{-1} H=0, M_{0} H=M_{1} H=\mathbb{Q} \mathbf{a}, M_{2} H=H .
$$

This result can also be stated by saying that the limit MHS on $H(1)$ of the variation $\mathbb{H}(1)$ associated to $z \partial / \partial q$ is the extension of $\mathbb{Z}(0)$ by $\mathbb{Z}(1)$ corresponding to $z \in \mathbb{C}^{*} \cong \operatorname{Ext}_{\mathrm{MHS}}^{1}(\mathbb{Z}(0), \mathbb{Z}(1))$.

Remark 9.8. Note that if $\vec{v} \in \mathbb{Q}^{\times} \partial / \partial q$, then $H_{\vec{v}}$ splits as an extension of $\mathbb{Q}(-1)$ by $\mathbb{Q}(0)$ if and only if $\vec{v}= \pm \partial / \partial q$. These are also the only two tangent vectors of the cusp $q=0$ of $\overline{\mathcal{M}}_{1,1}$ that are defined over $\mathbb{Z}$ and remain non-zero at every prime $p$. For this reason it is natural to identify the fiber $H$ of $\overline{\mathcal{H}}$ over $q=0$ with $H_{\partial / \partial q}$. In particular, $\mathbf{w}=-2 \pi i \mathbf{b}$.

These considerations suggest a natural choice of Cartan subalgebra of $\mathfrak{s l}(H)$ and of the positive root vectors. Namely, the Cartan is the one that acts diagonally with respect to the isomorphism

$$
H_{ \pm \partial / \partial q}=\mathbb{Q}(0) \oplus \mathbb{Q}(-1)=\mathbb{Q} \mathbf{a} \oplus \mathbb{Q} \mathbf{b} .
$$

The natural choice of a positive weight vector is the one with positive Hodge theoretic weight. Since $\mathbf{b}$ has Hodge weight 2 and $\mathbf{a}$ has weight 0 with respect to the weight filtration (9.7), the natural choice of positive root vectors in $\mathfrak{s l}(H)$ is $\mathbb{Q b} \partial / \partial \mathbf{a}$. This choice determines a Borel subalgebra of $\mathfrak{s l}(H)$.

With this choice of Cartan subalgebra, there are two natural choices of symplectic bases of $H_{\mathbb{Z}}$. Namely $\mathbf{a}, \mathbf{b}$ and $-\mathbf{b}, \mathbf{a}$. Because of formula (i) in Lemma 9.2 , which

\footnotetext{
${ }^{16}$ For the more arithmetically inclined, $\mathbf{a}, \mathbf{b}$ is a basis of the Betti component $H^{B}$ of the MHS $H$, and $\mathbf{a}, \mathbf{w}$ is a basis of the $\mathbb{Q}$-de Rham component $H^{\mathrm{DR}}$ of $H$.
} 
is dictated by the standard formula for the action of $\mathrm{SL}_{2}(\mathbb{Z})$ on $\mathfrak{h}$, we will use the basis $-\mathbf{b}, \mathbf{a}$. This choice determines corresponding isomorphisms $\mathfrak{s l}(H) \cong \mathfrak{s l}_{2}$ and $\mathrm{SL}(H) \cong \mathrm{SL}_{2}$. With respect to the above choice of Cartan subalgebra, $\mathbf{b}$ has $\mathfrak{s l}_{2}$-weight 1 and $\mathbf{a}$ has $\mathfrak{s l}_{2}$-weight -1 .

\section{Representation Theory OF $\mathrm{SL}_{2}$}

This is a quick review of the representation theory of $\mathfrak{s l}_{2}$ and $\mathrm{SL}_{2}$. Much of the time, $\mathrm{SL}_{2}$ will be $\mathrm{SL}(H)$, where $H=H_{\partial / \partial q}$ is the fiber of $\mathbb{H}$ over $\partial / \partial q$. As pointed out above, this has a natural basis, which leads us to a natural choice of Cartan and Borel subalgebras, which we make explicit below.

Let $F$ denote $\mathbb{Q}, \mathbb{R}$ or $\mathbb{C}$. Let $V$ be a two dimensional vector space over $F$ endowed with a symplectic form (i.e., a non-degenerate, skew symmetric bilinear form)

$$
\langle,\rangle: V \otimes V \rightarrow F .
$$

The choice of a symplectic basis $\mathbf{v}_{1}, \mathbf{v}_{2}$ of $V$ determines actions of $\mathrm{SL}_{2}(F)$ and $\mathfrak{s l}_{2}(F)$ on $V$ via the formula

$$
\left(\begin{array}{ll}
a & b \\
c & d
\end{array}\right):\left(\begin{array}{ll}
\mathbf{v}_{2} & \mathbf{v}_{1}
\end{array}\right)\left(\begin{array}{l}
x_{1} \\
x_{2}
\end{array}\right) \mapsto\left(\begin{array}{ll}
\mathbf{v}_{2} & \mathbf{v}_{1}
\end{array}\right)\left(\begin{array}{ll}
a & b \\
c & d
\end{array}\right)\left(\begin{array}{l}
x_{1} \\
x_{2}
\end{array}\right)
$$

where $x_{1}, x_{2} \in F$, and isomorphisms $\mathrm{SL}_{2} \cong \mathrm{SL}(V)$ and $\mathfrak{s l}_{2} \cong \mathfrak{s l}(H)$.

We will fix the choice of Cartan subalgebra of $\mathfrak{s l}_{2}$ to be the diagonal matrices. This fixes a choice of Cartan subalgebra of $\mathfrak{s l}(V)$. We will take $\mathbf{v}_{1}$ to have $\mathfrak{s l}_{2}$ weight -1 and $\mathbf{v}_{2}$ to have $\mathfrak{s l}_{2}$-weight +1 . The element

$$
\left(\begin{array}{ll}
0 & 1 \\
0 & 0
\end{array}\right)
$$

of $\mathfrak{s l}_{2}$ corresponds to $\mathbf{v}_{2} \partial / \partial \mathbf{v}_{1} \in \mathfrak{s l}(V)$ and has weight -2 . Denote it by $\mathbf{e}_{0}$.

Isomorphism classes of irreducible representations of $\mathrm{SL}(V)$ correspond to nonnegative integers. The integer $n \in \mathbb{N}$ corresponds to the $n$th symmetric power $S^{n} V$ of the defining representation $V$. To distinguish distinct but isomorphic representations of $\mathrm{SL}_{2}$, we use the notation

$$
S^{n}(\mathbf{e}):=\left\{\mathbf{e}_{0}^{j} \cdot \mathbf{e}: \mathbf{e}_{0}^{n+1} \cdot \mathbf{e}=0\right\}
$$

to denote the $\mathrm{SL}_{2}$-module with highest weight vector e of weigh $n$.

For example, motivated by the discussion in Remark 9.8, we will typically work in the following situation:

(i) $V=H$ and $\langle$, $\rangle$ is the intersection pairing,

(ii) $\mathbf{v}_{1}=-\mathbf{b}$ and $\mathbf{v}_{2}=\mathbf{a}$, so that $\mathbf{b}$ has $\mathfrak{s l}_{2}$ weight +1 and $\mathbf{a}$ has $\mathfrak{s l}_{2}$ weight -1 ,

(iii) $S^{n} H=S^{n}\left(\mathbf{b}^{n}\right)$,

(iv) $\mathbf{e}_{0}=-\mathbf{a} \frac{\partial}{\partial \mathbf{b}}$, which has $\mathfrak{s l}_{2}$ weight -2 . It is also $-2 \pi i$ times the residue at $q=0$ of the connection $\nabla_{0}$ on $\overline{\mathcal{H}}$.

Note that the weight filtration

$$
0=M_{-1} S^{n} H \subset M_{0} S^{n} H \subset \cdots \subset M_{2 n-1} S^{n} H \subset M_{2 n} S^{n} H=S^{n} H
$$

associated to the nilpotent endomorphism $\mathbf{e}_{0}$ of $S^{n} H$, shifted by the weight $n$ of $S^{n} H$, is the filtration obtained by giving $\mathbf{b}$ weight 2 and $\mathbf{a}$ weight 0 :

$$
M_{m} S^{n} H=\operatorname{span}\left\{\mathbf{a}^{n-j} \mathbf{b}^{j}: 2 j \leq m\right\},
$$


Observe that

$$
\mathrm{Gr}_{2 n}^{M} S^{n} H=S^{n} H / \mathrm{im} \mathbf{e}_{0} \cong \mathbb{Q}(-n) .
$$

It is generated by the highest weight vector $\mathbf{b}^{n}$.

\section{Modular Forms ANd Eichler-Shimura}

Suppose that $\Gamma$ is a finite index subgroup of $\mathrm{SL}_{2}(\mathbb{Z})$. Recall that a holomorphic function $f: \mathfrak{h} \rightarrow \mathbb{C}$ is a modular form of weight $w$ for $\Gamma$ if

(i) $f(\gamma \tau)=(c \tau+d)^{w} f(\tau)$ for all $\gamma \in \Gamma$. This implies that $f$ has a Fourier expansion $\sum_{k=-\infty}^{\infty} a_{k} q^{k / n}$ for some $n \geq 1$.

(ii) $f$ is holomorphic at each cusp $P \in \Gamma \backslash \mathbb{P}^{1}(\mathbb{Q})$. If, for example, $P=\infty$, this means that the coefficients $a_{k}$ of the Fourier expansion of $f$ at $P$ vanish when $k<0$.

A modular form $f$ is a cusp form if it vanishes at each cusp - that is, its Fourier coefficients $a_{k}$ vanish for all $k \leq 0$.

Assume the notation of Section 10. For an indeterminate e we have the $\mathrm{SL}_{2}$ module $S^{m}(\mathbf{e})$ that is isomorphic to $S^{m} H$. Denote the corresponding local system over $X_{\Gamma}$ by $\mathbb{S}^{m}(\mathbf{e})$. As a local system, it is isomorphic to $S^{m} \mathbb{H}$. Lemma 7.1 implies that if $\mathbb{S}^{m}(\mathbf{e})$ has the structure of a PVHS, then it is isomorphic to $S^{m} \mathbb{H}(r)$ for some $r \in \mathbb{Z}$.

For the time being, we will suppose that the PVHS $\mathbb{S}^{m}(\mathbf{e})$ over $X_{\Gamma}$ has weight $m$, so that it is an isomorphic copy of $S^{m} \mathbb{H}$. Define a function $v: \mathfrak{h} \rightarrow V$ by

$$
v(\tau, \mathbf{e}):=\exp \left(\tau \mathbf{e}_{0}\right) \mathbf{e}
$$

The discussion preceding Lemma 9.2 implies that $v(\tau, \mathbf{e})$ is a trivializing section of $F^{m}\left(\mathbb{S}^{m}(\mathbf{e}) \otimes \mathcal{O}_{\mathfrak{h}}\right)$.

Lemma 11.1. For all $\gamma \in \mathrm{SL}_{2}(\mathbb{Z})$ we have $(c \tau+d)^{m} \gamma^{*} v=\gamma_{*} v$.

Proof. Write $\mathbf{e}=\mathbf{b}^{m}$, where $\mathbf{b} \in H$. Then $\exp \left(\tau \mathbf{e}_{0}\right) \mathbf{e}=\left(\exp \left(\tau \mathbf{e}_{0}\right) \mathbf{b}\right)^{m}$. So it suffices to consider the case $m=1$. In this case $v(\tau, \mathbf{b})=(-\mathbf{a}, \mathbf{b})(\tau, 1)^{T}$ and

$$
\left(\gamma_{*} v\right)(\tau, \mathbf{b})=-\left(\begin{array}{ll}
\mathbf{a} & -\mathbf{b}
\end{array}\right)\left(\begin{array}{ll}
a & b \\
c & d
\end{array}\right)\left(\begin{array}{l}
\tau \\
1
\end{array}\right)=-(c \tau+d)\left(\begin{array}{ll}
\mathbf{a} & -\mathbf{b}
\end{array}\right)\left(\begin{array}{c}
\gamma \tau \\
1
\end{array}\right)=\left(\gamma^{*} v\right)(\tau, \mathbf{b})
$$

For a modular form $f$ of $\Gamma$ weight $w=m+2$ and an indeterminate e, define ${ }^{17}$

$$
\omega_{f}(\mathbf{e})=2 \pi i f(\tau) v(\tau, \mathbf{e}) d \tau \in E^{1}(\mathfrak{h}) \otimes S^{m}(\mathbf{e}) .
$$

A routine calculation shows that $\omega_{f}(\mathbf{e})$ is $\Gamma$-invariant in the sense that

$$
\left(\gamma^{*} \otimes 1\right) \omega_{f}(\mathbf{e})=(1 \otimes \gamma) \omega_{f}(\mathbf{e})
$$

It follows that

$$
\omega_{f}(\mathbf{e}) \in\left(E^{1}(\mathfrak{h}) \otimes S^{m}(\mathbf{e})\right)^{\Gamma} \cong E^{1}\left(X_{\Gamma}, \mathbb{S}^{m}(\mathbf{e})\right) .
$$

Since $\omega_{f}(\mathbf{e})$ is closed, it determines a class in $H^{1}\left(X_{\Gamma}, \mathbb{S}^{m}(\mathbf{e})\right)$. Its complex conjugate

$$
\bar{\omega}_{f}(\mathbf{e})=\overline{f(\tau)} v(\bar{\tau}, \mathbf{e})^{m} d \bar{\tau}
$$

\footnotetext{
${ }^{17}$ The particular scaling by $2 \pi i$ is chosen so that, when $\Gamma=\mathrm{SL}_{2}(\mathbb{Z})$, if $v(\tau, \mathbf{e})$ corresponds (locally) to a $\mathbb{Q}$-rational section of the canonical extension $S^{m} \mathcal{H}(r)$ of $S^{m} \mathbb{H}$ to $\overline{\mathcal{M}}_{1,1}$, then $\omega_{f}$ is $\mathbb{Q}$-rational in the sense that $\omega_{f}(\mathbf{e}) \in H_{\mathrm{DR}}^{1}\left(\mathcal{M}_{1,1 / \mathbb{Q}}, S^{m} \mathcal{H}\right)$. See, e.g., $[20, \S 21]$.
} 
also defines a class in $H^{1}\left(X_{\Gamma}, \mathbb{S}^{m}(\mathbf{e})\right)$.

Recall that $X_{\Gamma}=\bar{X}_{\Gamma}-D$, where $D=\Gamma \backslash \mathbb{P}^{1}(\mathbb{Q})$. And recall from Section 6 that $K^{\bullet}\left(\bar{X}, D ; \mathbb{S}^{n}(\mathbf{e})\right)$ is Zucker's mixed Hodge complex that computes the MHS on $H^{\bullet}\left(X_{\Gamma}, \mathbb{S}^{n}(\mathbf{e})\right)$. It is straightforward to check that:

Proposition 11.2 (Zucker [45]). If $f$ is a modular form of $\Gamma$ of weight $w=m+2$, then

$$
\omega_{f}(\mathbf{e}) \in F^{m+1} K^{1}\left(\bar{X}_{\Gamma}, D ; \mathbb{S}^{m}(\mathbf{e})\right) .
$$

If $f$ is cusp form, then $\omega_{f}(\mathbf{e}) \in F^{m+1} W_{m} K^{1}\left(\bar{X}_{\Gamma}, D ; \mathbb{S}^{m}(\mathbf{e})\right)$.

When $m>0$ the exact sequence (6.3) from Section 6.4 becomes

$$
0 \rightarrow W_{m+1} H^{1}\left(X_{\Gamma}, \mathbb{S}^{m}(\mathbf{e})\right) \rightarrow H^{1}\left(X_{\Gamma}, \mathbb{S}^{m}(\mathbf{e})\right) \rightarrow \bigoplus_{P \in D}\left(\left(S^{m} V_{P}\right) / \operatorname{im} N_{P}\right)(-1) \rightarrow 0,
$$

where $V_{P}$ denotes the fiber of the canonical extension of $\mathbb{S}^{m}(\mathbf{e}) \otimes \mathcal{O}_{X_{\Gamma}}$ to $C$ and $N_{P}$ the associated local monodromy operator. (Cf. Section 6.2.) It is an exact sequence of MHS. Note that each $S^{m} V_{P} / \operatorname{im} N_{P}$ is one dimensional and is isomorphic to $\mathbb{Q}(-m)$ by $(10.2)$.

The following result is equivalent to Eichler-Shimura combined with the observations that

$$
K^{1}\left(\bar{X}_{\Gamma}, D ; \mathbb{S}^{m}(\mathbf{e})\right)=W_{2 m+1} K^{1}\left(\bar{X}_{\Gamma}, D ; \mathbb{S}^{m}(\mathbf{e})\right) \text { and } F^{m+2} K^{1}\left(\bar{X}_{\Gamma}, D ; \mathbb{S}^{m}(\mathbf{e})\right)=0 .
$$

This version was proved by Zucker in [45].

Denote the space of modular forms of $\Gamma$ of weight $w$ by $\mathfrak{M}_{w}(\Gamma)$ and the subspace of cusp forms by $\mathfrak{M}_{w}^{o}(\Gamma)$. When $\Gamma=\mathrm{SL}_{2}(\mathbb{Z})$, we will omit $\Gamma$ and simply write $\mathfrak{M}_{w}$ and $\mathfrak{M}_{w}^{o}$.

Theorem 11.4 (Shimura, Zucker). If $\Gamma$ is a finite index subgroup of $\mathrm{SL}_{2}(\mathbb{Z})$, then $H^{1}\left(X_{\Gamma}, \mathbb{S}^{m}(\mathbf{e})\right)$ is spanned by the classes of modular forms of weight $w=m+2$ and their complex conjugates. The only non-vanishing Hodge numbers $h^{p, q}$ occur when $(p, q)=(m+1,0),(0, m+1)$ and $(m+1, m+1)$. The weight $m+1$ part is spanned by the classes of cusp forms and their complex conjugates. Moreover, the function that takes a modular form $f$ to the class of $\omega_{f}(\mathbf{e})$ induces an isomorphism

$$
\mathfrak{M}_{m+2}(\Gamma) \cong F^{m+1} H^{1}\left(X_{\Gamma}, \mathbb{S}^{m}(\mathbf{e})\right) .
$$

The map that takes a cusp form $f$ to the class of its complex conjugate $\bar{\omega}_{f}(\mathbf{e})$ induces a conjugate linear isomorphism of $\mathfrak{M}_{k+2}^{o}(\Gamma)$ with the $(0, m+1)$ part of the Hodge structure $W_{m+1} H^{1}\left(X_{\Gamma}, \mathbb{S}^{m}(\mathbf{e})\right)$.

When $\Gamma=\mathrm{SL}_{2}(\mathbb{Z})$, there is only one cusp. It is routine to show that the cohomology with coefficients in $\mathbb{S}^{m}(\mathbf{e})$ vanishes when $m$ is odd. So for each $n>0$ we have the exact sequence

$$
0 \rightarrow W_{2 n+1} H^{1}\left(\mathcal{M}_{1,1}, \mathbb{S}^{2 n}(\mathbf{e})\right) \rightarrow H^{1}\left(\mathcal{M}_{1,1}, \mathbb{S}^{2 n}(\mathbf{e})\right) \rightarrow \mathbb{Q}(-2 n-1) \rightarrow 0
$$

The class of the 1-form $\omega_{f}(\mathbf{e})$ associated to the Eisenstein series $f=G_{2 n+2}$ of weight $2 n+2$ projects to a generator of $\mathbb{Q}(-2 n-1)$. The MHS on $H^{1}\left(X_{\Gamma}, \mathbb{S}^{2 n}(\mathbf{e})\right)$ can be described in terms of modular symbols. We will return to this in Section 17.2. 
11.1. Cohomology of congruence subgroups. Recall that a congruence subgroup of $\mathrm{SL}_{2}(\mathbb{Z})$ is one that contains a principal congruence subgroup

$$
\mathrm{SL}_{2}(\mathbb{Z})[N]:=\left\{\gamma \in \mathrm{SL}_{2}(\mathbb{Z}): \gamma \equiv \text { identity } \bmod N\right\} .
$$

When $\Gamma$ is a congruence subgroup, one has Hecke operators

$$
T_{p} \in \operatorname{End}_{\mathrm{MHS}} H^{1}\left(X_{\Gamma}, S^{m} \mathbb{H}\right)
$$

for each prime number $p$. Since the Hecke algebra (the algebra generated by the $\left.T_{p}\right)$ is semi-simple, $H^{1}\left(X_{\Gamma}, S^{m} \mathbb{H}_{\mathbb{Q}}\right)$ decomposes into simple factors. Each is a MHS.

Let $\mathfrak{B}_{w}(\Gamma)$ be the set of normalized Hecke eigen cusp forms of $\Gamma$ of weight $w \geq 2$. This is a basis of $\mathfrak{M}_{w}^{o}(\Gamma)$, the weight $w$ cusp forms. For each $f \in \mathfrak{B}_{w}(\Gamma)$, let $K_{f}$ be the subfield of $\mathbb{C}$ generated by its Fourier coefficients. Since the restriction of $T_{p}$ to the cusp forms is self adjoint with respect to the Petersson inner product, its eigenvalues are real. Since this holds for all $p$, it implies that $K_{f} \subset \mathbb{R}$. Consequently, the smallest subspace $V_{f}$ of $H^{1}\left(X_{\Gamma}, S^{m} \mathbb{H}_{K_{f}}\right)$ whose complexification contains $\omega_{f}(\mathbf{e})$ is a $K_{f}$-sub HS of the MHS $H^{1}\left(X_{\Gamma}, S^{m} \mathbb{H}\right)$.

Denote by $M_{f}$ the smallest $\mathbb{Q}$-Hodge sub-structure of $H^{1}\left(X_{\Gamma}, S^{m} \mathbb{H}_{\mathbb{Q}}\right)$ with the property that $M_{f} \otimes K_{f}$ contains $V_{f}$. It is a sum of the Hodge structures $V_{h}$ of the eigenforms conjugate to $f$. Call two eigenforms $f$ and $h$ equivalent if $M_{f}=M_{h}$.

When $f$ is a normalized Eisenstein series the smallest sub-MHS of $H^{1}\left(X_{\Gamma}, S^{m} \mathbb{H}_{\mathbb{Q}}\right)$ that contains the corresponding cohomology class is one dimensional and spans a Tate MHS $\mathbb{Q}(-m-1)$.

Theorem 11.5. If $\Gamma$ is a congruence subgroup of $\mathrm{SL}_{2}(\mathbb{Z})$, then the MHS Hodge structure on $H^{1}\left(X_{\Gamma}, S^{m} \mathbb{H}\right)$ splits. In particular, when $m>0$, there is a canonical isomorphism

$$
H^{1}\left(X_{\Gamma}, S^{m} \mathbb{H}\right) \cong \bigoplus_{f} M_{f} \oplus \bigoplus_{P \in D} \mathbb{Q}(-m-1),
$$

where $f$ ranges over the equivalence classes of eigen cusp forms of weight $m+2$. As a real mixed Hodge structure

$$
H^{1}\left(X_{\Gamma}, S^{m} \mathbb{H}\right) \cong \bigoplus_{f \in \mathfrak{B}_{m+2}(\Gamma)} V_{f} \oplus \bigoplus_{P \in D} \mathbb{R}(-m-1) .
$$

Sketch of Proof. The splitting of the MHS on $H^{1}\left(X_{\Gamma}, S^{m} \mathbb{H}\right)$ follows from the fact that each Hecke operator $T_{p}$ is a morphism of MHS. The weight filtration splits because $T_{p}$ acts on $\mathrm{Gr}_{m+1}^{W}$ with eigenvalues of modulus bounded by $C p^{1+m / 2}$ and on $\mathrm{Gr}_{2 m+2}^{W}$ with eigenvalues of size $O\left(p^{m+1}\right)$. (For $\mathrm{SL}_{2}(\mathbb{Z})$ this is proved in [38]. See p. 94 and p. 106 . For general $\Gamma$ see $\left[27\right.$, Lem. 2, p. 13].) The $V_{f}$ are the common eigenspaces of the $T_{p}$ acting on $W_{m+1} H^{1}\left(X_{\Gamma}, S^{m} \mathbb{H}\right)$. The $M_{f}$ are their Galois orbits and are $\mathbb{Q}$-HS.

\section{Hodge Theory of the Relative Completion of Modular Groups}

Here we make the construction of the mixed Hodge structure on the unipotent radical of the completion of a modular group with respect to its inclusion into $\mathrm{SL}_{2}(\mathbb{Q})$ explicit.

We retain the notation of previous sections: $\Gamma$ is a finite index subgroup of $\mathrm{SL}_{2}(\mathbb{Z}), X_{\Gamma}=\Gamma \backslash \mathfrak{h}$ is the associated curve, $D=\Gamma \backslash \mathbb{P}^{1}(\mathbb{Q})$ is the set of cusps, and $\bar{X}_{\Gamma}=X_{\Gamma} \cup D$ is its smooth completion. As in Section $9, H$ denotes the fiber over the unique cusp $q=0$ of $\overline{\mathcal{M}}_{1,1}$ of the canonical extension $\overline{\mathcal{H}}$ of the local system 
$\mathbb{H}$. The pullback of $\overline{\mathcal{H}}$ along the quotient morphism $\bar{X}_{\Gamma} \rightarrow \overline{\mathcal{M}}_{1,1}$ is the canonical extension of $\mathbb{H} \otimes \mathcal{O}_{X_{\Gamma}}$ to $\bar{X}_{\Gamma}$, so that the fiber of $\overline{\mathcal{H}}_{\bar{X}_{\Gamma}}$ over each $P \in D$ is naturally isomorphic to $H$.

Fix a base point $x_{o}$ of $X_{\Gamma}$. We allow $x_{o}$ to be a non-zero tangent vector at a cusp $P \in D$. Denote the completion of $\pi_{1}\left(X_{\Gamma}, x_{o}\right)$ with respect to its inclusion into $\mathrm{SL}_{2}(\mathbb{Q})$ by $\mathcal{G}_{x_{o}}$ and its prounipotent radical by $\mathcal{U}_{x_{o}}$. Their Lie algebras (and coordinate rings) have natural mixed Hodge structures. Recall that the polarized variation $\mathbb{H}$ over $X_{\Gamma}$ has weight 1 . Denote its fiber over $x_{o}$ by $H_{o}$.

We also fix a lift $\tau_{o}$ of $x_{o}$ to $\mathfrak{h}$. This determines an isomorphism $\pi_{1}\left(X_{\Gamma}, x_{o}\right) \cong \Gamma$ and isomorphisms of $\mathcal{G}_{x_{o}}$ and $\mathcal{U}_{x_{o}}$ with the completion $\mathcal{G}$ of $\Gamma$ with respect to the inclusion $\Gamma \hookrightarrow \mathrm{SL}_{2}(\mathbb{Q})$, and $\mathcal{U}$, its prounipotent radical.

12.1. General considerations. As pointed out in Section $3.4 .2, \mathfrak{u}$ is free. So, up to a non-canonical isomorphism, it is determined by its abelianization $H_{1}(\mathfrak{u})$. Theorem 7.15, implies that $\mathfrak{u}$ has negative weights, so there is an exact sequence

$$
0 \rightarrow W_{-2} H_{1}(\mathfrak{u})_{\text {eis }} \rightarrow H_{1}(\mathfrak{u}) \rightarrow \mathrm{Gr}_{-1}^{W} \mathfrak{u} \rightarrow 0 .
$$

of pro-MHS with $\mathrm{SL}_{2}$ action. Eichler-Shimura (Thm. 11.4) and the computation (3.4) imply that the weight -1 quotient comes from cusp forms:

$$
\operatorname{Gr}_{-1}^{W} \mathfrak{u}=\prod_{m \geq 0}\left(W_{m+1} H^{1}\left(X_{\Gamma}, S^{m} \mathbb{H}\right)\right)^{*} \otimes S^{m} H_{o}=\prod_{m \geq 0} I H^{1}\left(X_{\Gamma}, S^{m} \mathbb{H}\right)^{*} \otimes S^{m} H_{o}
$$

The exact sequence (6.3) implies that the weight $<-1$ part

$$
W_{-2} H_{1}(\mathfrak{u})=\widetilde{H}_{0}(D ; \mathbb{Q}(1)) \oplus \bigoplus_{P \in D} \prod_{m \geq 0} S^{m} H_{o}(m+1)
$$

is a direct product of Hodge structures. Note that when $x_{o}$ is a finite base point (i.e., $\left.x_{o} \in X_{\Gamma}\right)$, then $S^{m} H_{o}(m+1)$ has weight $-m-1$. If $x_{o}$ is a tangent vector at a cusp, then $S^{m} H_{o}(m+1)$ has weight graded quotients $\mathbb{Q}(1), \mathbb{Q}(2), \ldots, \mathbb{Q}(m+1)$. In this case, $W_{-2} H_{1}(\mathfrak{u})$ is mixed Tate.

The Manin-Drinfeld Theorem (Thm. 11.5) implies:

Proposition 12.1. If $\Gamma$ is a congruence subgroup of $\mathrm{SL}_{2}(\mathbb{Z})$, then $H_{1}(\mathfrak{u})$ is the product

$$
H_{1}(\mathfrak{u}) \cong \prod_{r<0} \mathrm{Gr}_{r}^{W} H_{1}(\mathfrak{u})
$$

of its weight graded quotients in the category of pro-MHS with $\mathrm{SL}_{2}$ action.

12.2. Hodge theory of congruence subgroups. Now assume that $\Gamma$ is a congruence subgroup of $\mathrm{SL}_{2}(\mathbb{Z})$. The first step in writing down a formal connection $\Omega \in K^{1}\left(\bar{X}_{\Gamma}, D ; \mathfrak{u}\right)$ is to write down, for each $m>0$, a form

$$
\Omega_{1, m} \in F^{0} W_{-1}\left(K^{1}\left(\bar{X}_{\Gamma}, D ; S^{m} \mathbb{H}\right) \otimes H^{1}\left(X_{\Gamma}, S^{m} \mathbb{H}\right)^{*}\right)
$$

that represents the identity $H^{1}\left(X_{\Gamma}, S^{m} \mathbb{H}\right) \rightarrow H^{1}\left(X_{\Gamma}, S^{m} \mathbb{H}\right)$.

Since $\Gamma$ is a congruence subgroup, the Hecke algebra acts on the modular forms $\mathfrak{M}_{w}(\Gamma)$ of $\Gamma$ of weight $w$. When $w>2$, for each cusp $P \in D$, there is a normalized Eisenstein series $E_{w, P}(\tau)$ that vanishes at the other cusps. When $w=2$, Eisenstein series give elements of $H^{1}\left(X_{\Gamma}, \mathbb{C}\right)$ with non-zero residues at least two cusps. Fix a cusp $P_{o} \in D$. For each $P \in D^{\prime}:=D-\left\{P_{o}\right\}$, choose an Eisenstein series $E_{2, P}$ that is non-zero at $P$ and vanishes at all other points of $D^{\prime}$. 
Now suppose that $m \geq 0$. Identify $S^{m} \mathbb{H}$ with $\mathbb{S}^{m}\left(\mathbf{b}^{m}\right)$. For each $f \in \mathfrak{B}_{m+2}(\Gamma)$ we have the 1-forms $\omega_{f}\left(\mathbf{b}^{m}\right)$ and $\bar{\omega}_{f}\left(\mathbf{b}^{m}\right)$. When $m>0$ (resp. $m=0$ ) and $P \in D$ (resp. $P \in D^{\prime}$ ) set

$$
\psi_{m, P}\left(\mathbf{b}^{m}\right):=\omega_{E_{m, P}}\left(\mathbf{b}^{m}\right) .
$$

This will be viewed as an element of $K^{1}\left(\bar{X}_{\Gamma}, D ; S^{m} \mathbb{H}\right)$ and of $E^{1}\left(\mathfrak{h}, S^{n} H\right)^{\Gamma}$. Then

$$
\begin{aligned}
\left\{\omega_{f}\left(\mathbf{b}^{m}\right), \bar{\omega}_{f}\left(\mathbf{b}^{m}\right): f \in \mathfrak{B}_{m+2}(\Gamma)\right\} \cup & \\
& \left\{\psi_{m+2, P}\left(\mathbf{b}^{m}\right):(P \in D \text { and } m>0) \text { or }\left(P \in D^{\prime} \text { and } m=0\right)\right\}
\end{aligned}
$$

is a subset of $K^{1}\left(\bar{X}_{\Gamma}, D ; S^{m} \mathbb{H}\right)$ that represents a basis of $H^{1}\left(X_{\Gamma}, S^{m} \mathbb{H}\right)$. Let

$$
\left\{u_{f}^{\prime}, u_{f}^{\prime \prime}, u_{m+2, P}: f \in \mathfrak{B}_{m+2}(\Gamma), P \in D\right\}
$$

be a basis of $H^{1}\left(X_{\Gamma}, S^{m} \mathbb{H}\right)$ dual to the cohomology classes of the closed forms (12.2). The Hodge types of $u_{f}^{\prime}, u_{f}^{\prime \prime}$ and $u_{m+2, P}$ are $(-m-1,0),(0,-m-1)$ and $(-m-1,-m-1)$, respectively

Set

$$
\mathbf{e}_{f}^{\prime}:=\mathbf{b}^{m} \otimes u_{f}, \mathbf{e}_{f}^{\prime \prime}:=\mathbf{b}^{m} \otimes \bar{u}_{f}, \mathbf{e}_{m+2, P}=\mathbf{b}^{m} \otimes u_{m+2, P} .
$$

These are elements of $S^{m} H \otimes H^{1}\left(X_{\Gamma}, S^{m} \mathbb{H}\right)^{*}$. Then

$$
\begin{aligned}
& \omega_{f}\left(\mathbf{b}^{m}\right) \otimes u_{f}^{\prime}=\omega_{f}\left(\mathbf{e}_{f}^{\prime}\right), \bar{\omega}_{f}\left(\mathbf{b}^{m}\right) \otimes u_{f}^{\prime \prime}=\bar{\omega}_{f}\left(\mathbf{e}_{f}^{\prime \prime}\right), \\
& \quad \text { and } \psi_{m+2, P}\left(\mathbf{b}^{m}\right) \otimes u_{m+2, P}=\psi_{m+2, P}\left(\mathbf{e}_{m+2, P}\right) .
\end{aligned}
$$

All are elements of $K^{1}\left(\bar{X}_{\Gamma}, D ; S^{m} \mathbb{H}\right) \otimes H^{1}\left(X_{\Gamma}, S^{m} \mathbb{H}\right)^{*}$ and

$$
\Omega_{1, m}=\sum_{f \in \mathfrak{B}_{m+2}(\Gamma)}\left(\omega_{f}\left(\mathbf{e}_{f}^{\prime}\right)+\bar{\omega}_{f}\left(\mathbf{e}_{f}^{\prime \prime}\right)\right)+\sum_{P} \psi_{m+2, P}\left(\mathbf{e}_{m+2, P}\right)
$$

is a closed 1-form that represents the identity $H^{1}\left(X_{\Gamma}, S^{m} \mathbb{H}\right) \rightarrow H^{1}\left(X_{\Gamma}, S^{m} \mathbb{H}\right)$. Here the second sum is over $P \in D$ when $m>0$ and $P \in D^{\prime}$ when $m=0$.

Lemma 12.5. For each $m \geq 0$

$$
\Omega_{1, m} \in F^{0} W_{-1}\left(K^{1}\left(\bar{X}_{\Gamma}, D ; S^{m} \mathbb{H}\right) \otimes H^{1}\left(X_{\Gamma}, S^{m} \mathbb{H}\right)^{*}\right) .
$$

Proof. Since the Hodge types of $u_{f}^{\prime}, u_{f}^{\prime \prime}$ and $u_{m+2, P}$ are $(-m-1,0),(0,-m-1)$ and $(-m-1,-m-1)$, the definitions of the Hodge and weight filtrations of Zucker's mixed Hodge complex $K^{\bullet}\left(\bar{X}_{\Gamma}, D ; S^{m} \mathbb{H}\right)$ imply that

$$
\omega_{f}\left(\mathbf{b}^{m}\right) \in F^{m+1} W_{m} K^{1}\left(\bar{X}_{\Gamma}, D ; S^{m} \mathbb{H}\right) \text { and } \bar{\omega}_{f}\left(\mathbf{b}^{m}\right) \in F^{0} W_{m} K^{1}\left(\bar{X}_{\Gamma}, D ; S^{m} \mathbb{H}\right)
$$

when $f \in \mathfrak{B}_{m+2}(\Gamma)$ and $\psi_{m+2, P}\left(\mathbf{b}^{m}\right) \in F^{m+1} W_{2 m+1} K^{1}\left(\bar{X}_{\Gamma}, D ; S^{m} \mathbb{H}\right)$ for each $P$. The result follows as $u_{f}^{\prime}, u_{f}^{\prime \prime}$ and $u_{m+2, P}$ have Hodge types $(-m-1,0),(0,-m-1)$ and $(-m-1,-m-1)$, respectively.

The Lie algebra $\mathfrak{u}$ of the prounipotent radical $\mathcal{U}$ of the relative completion of $\Gamma$ is the free pronilpotent Lie algebra $\mathfrak{u}=\mathbb{L}(V)^{\wedge}$, where $V=\bigoplus_{m \geq 0} V_{m}$ and

$$
\begin{aligned}
V_{m} & :=H^{1}\left(X_{\Gamma}, S^{m} \mathbb{H}\right)^{*} \otimes S^{m} H \\
& = \begin{cases}H_{1}\left(X_{\Gamma}, \mathbb{C}\right) & m=0, \\
\bigoplus_{f \in \mathfrak{B}_{m+2}(\Gamma)}\left(S^{m}\left(\mathbf{e}_{f}^{\prime}\right) \oplus S^{m}\left(\mathbf{e}_{f}^{\prime \prime}\right)\right) \oplus \bigoplus_{P \in D} S^{m}\left(\mathbf{e}_{m+2, P}\right) & m>0 .\end{cases}
\end{aligned}
$$

It is a Lie algebra in the category of pro-representations of $\mathrm{SL}_{2}$. 
The 1-form

$$
\Omega_{1}:=\sum_{m \geq 0} \Omega_{1, m} \in E^{1}(\mathfrak{h}) \hat{\otimes} \mathfrak{u}
$$

is $\Gamma$-invariant and represents the identity. It can thus be completed to a power series connection

$$
\Omega \in F^{0} W_{-1} K^{1}\left(\bar{X}_{\Gamma}, D ; \mathfrak{u}\right)
$$

using the method described in Section 7, which determines the MHS on $\mathfrak{u}_{x_{o}}$.

Before discussing the case $\Gamma=\mathrm{SL}_{2}(\mathbb{Z})$, note that since

$$
\begin{array}{rlrl}
S^{m}\left(\mathbf{e}_{f}^{\prime}\right) & =\operatorname{span}\left\{\mathbf{e}_{0}^{j} \cdot \mathbf{e}_{f}^{\prime}: \mathbf{e}_{0}^{m+1} \cdot \mathbf{e}_{f}^{\prime}=0\right\}, & & f \in \mathfrak{B}_{m+2}(\Gamma) \\
S^{m}\left(\mathbf{e}_{f}^{\prime \prime}\right) & =\operatorname{span}\left\{\mathbf{e}_{0}^{j} \cdot \mathbf{e}_{f}^{\prime \prime}: \mathbf{e}_{0}^{m+1} \cdot \mathbf{e}_{f}^{\prime \prime}=0\right\}, & & f \in \mathfrak{B}_{m+2}(\Gamma) \\
S^{m}\left(\mathbf{e}_{m+2, P}\right) & =\operatorname{span}\left\{\mathbf{e}_{0}^{j} \cdot \mathbf{e}_{m+2, P}: \mathbf{e}_{0}^{m+1} \cdot \mathbf{e}_{m+2, P}=0\right\}, & P \in D,
\end{array}
$$

$\mathfrak{u}$ is the free Lie algebra topologically generated by

$\left\{\mathbf{e}_{2, P}: P \in D^{\prime}\right\} \cup \bigcup_{m>0}\left\{\mathbf{e}_{0}^{j} \cdot \mathbf{e}_{f}^{\prime}, \mathbf{e}_{0}^{j} \cdot \mathbf{e}_{f}^{\prime \prime}, \mathbf{e}_{0}^{j} \cdot \mathbf{e}_{P, m+2}: 0 \leq j \leq m, f \in \mathfrak{B}_{m+2}, P \in D\right\}$.

The Hodge and weight filtrations of $\mathfrak{u}$ are defined by giving $\mathbf{b} \in H$ type $(1,0)$. The generators (12.3) thus have types given in Figure 4 . So, for example, $\mathbf{e}_{0}^{j} \cdot \mathbf{e}_{f}^{\prime}$ has type

\begin{tabular}{|c|c|c|c|c|}
\hline & & Hodge type & $W$-weight & $M$-weight \\
\hline $\mathbf{e}_{0}$ & & $(-1,1)$ & 0 & -2 \\
$\mathbf{e}_{f}^{\prime}$ & $f \in \mathfrak{B}_{m+2}(\Gamma)$ & $(-1,0)$ & -1 & $m-1$ \\
$\mathbf{e}_{f}^{\prime \prime}$ & $f \in \mathfrak{B}_{m+2}(\Gamma)$ & $(m,-m-1)$ & -1 & $m-1$ \\
$\mathbf{e}_{m+2, P}$ & $P \in D$ & $(-1,-m-1)$ & $-m-2$ & -2 \\
\hline
\end{tabular}

Figure 4. Hodge types of the generators of $\mathfrak{u}$

$(-1-j, j)$. The Hodge and weight filtrations on the generators extend naturally to Hodge and weight filtrations on $\mathfrak{u}$.

The Hodge types on the $\mathfrak{s l}_{2}$ module with highest weight vectors $\mathbf{e}_{f}^{\prime}, \mathbf{e}_{f}^{\prime \prime}, \mathbf{e}_{m+2, P}$ are illustrated in Figure 5.

12.3. The case of $\mathrm{SL}_{2}(\mathbb{Z})$. In this case, $X_{\Gamma}=\mathcal{M}_{1,1}, C=\overline{\mathcal{M}}_{1,1}$ and $D$ consists of a single point, which we shall denote by $P$. The modular parameter $q=e^{2 \pi i \tau}$ is a local holomorphic coordinate on the orbifold $\overline{\mathcal{M}}_{1,1}$ centered at $P$.

There are no modular forms for $\mathrm{SL}_{2}(\mathbb{Z})$ of odd weight. Since there is a single cusp, there is a 1-dimensional space of Eisenstein series for each weight $2 n \geq 4$. The normalized Eisenstein series of even weight $2 n$ is

$$
G_{2 n}(\tau)=\frac{1}{2} \frac{(2 n-1) !}{(2 \pi i)^{2 n}} \sum_{\substack{\lambda \in \mathbb{Z} \oplus \mathbb{Z} \tau \\ \lambda \neq 0}} \frac{1}{\lambda^{2 n}}=-\frac{B_{2 n}}{4 n}+\sum_{k=1}^{\infty} \sigma_{2 n-1}(k) q^{k} .
$$

This has value $(2 n-1) ! \zeta(2 n) /(2 \pi i)^{2 n}$ at the cusp $P .{ }^{18}$ The dual homology class $\mathbf{e}_{2 n, P}$ will be denoted by $\mathbf{e}_{2 n}$ and the form $\psi_{2 n, P}$ by $\psi_{2 n}$.

Later, we will use the tangent vector $\vec{v}:=\partial / \partial q$ of $P$ as a base point. We will also write $\mathfrak{B}_{w}$ instead of $\mathfrak{B}_{w}\left(\mathrm{SL}_{2}(\mathbb{Z})\right)$.

\footnotetext{
${ }^{18}$ Here $B_{2 n}$ is the $2 n$th Bernoulli number, $\zeta(s)$ is the Riemann zeta function and $\sigma_{k}(n)$ is the sum of the $k$ th powers of the divisors of $n$.
} 


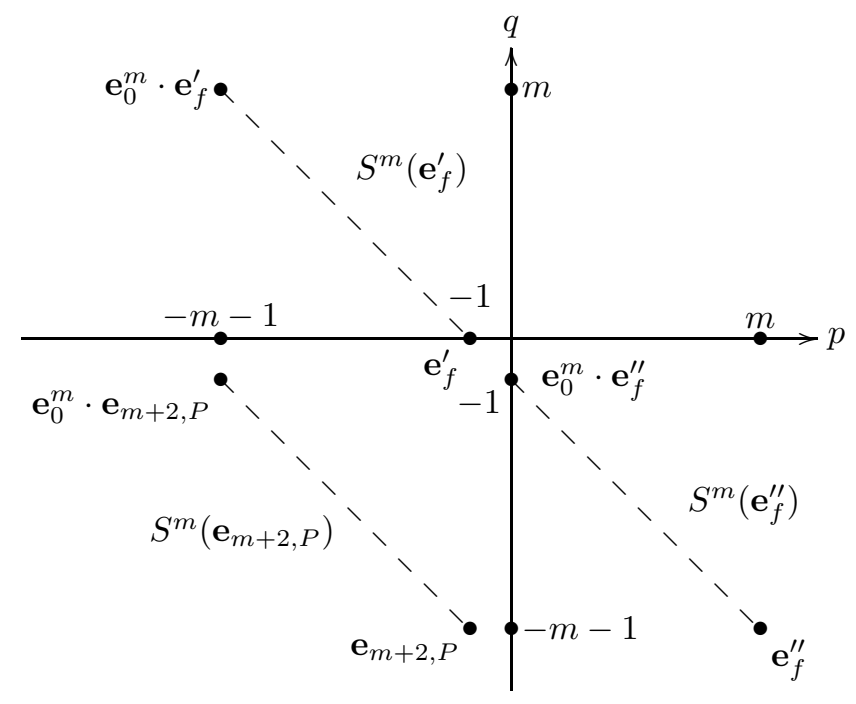

Figure 5. Hodge numbers of $S^{m}\left(\mathbf{e}_{f}^{\prime}\right), S^{m}\left(\mathbf{e}_{f}^{\prime \prime}\right)$ and $S^{m}\left(\mathbf{e}_{m+2, P}\right)$.

\section{VMHS associated to Modular Forms and their Period Maps}

This section considers three related topics: relative higher albanese maps (which are related to period mappings of VMHS), Manin's iterated Shimura integrals, and the existence of extensions of VMHS coming from Hecke eigenforms. The construction of these extensions is a special case of the general technique for constructing admissible VMHS sketched in the proof of Theorem 8.3. Such extensions correspond to normal functions, so every Hecke eigenform produces a normal function.

13.1. Relative Albanese maps. This construction generalizes the non-abelian albanese manifolds of [25] from the unipotent case to the relative case. Although this discussion applies more generally, here we restrict to the case of modular curves.

Fix a base point $\tau_{o}$ of $\mathfrak{h}$. Let $\Gamma$ be a finite index subgroup of $\mathrm{SL}_{2}(\mathbb{Z})$. Denote the image of $\tau_{o}$ in $X_{\Gamma}$ by $x_{o}$. The choice of $\tau_{o}$ determines an isomorphism $\Gamma \cong$ $\pi_{1}\left(X_{\Gamma}, x_{o}\right)$. Let $\mathcal{G}_{o}$ be the complex form of the relative completion of $\pi_{1}\left(X_{\Gamma}, x_{o}\right)$. Let $\mathcal{U}_{o}$ be its prounipotent radical, and let $\mathfrak{g}_{o}, \mathfrak{u}_{o}$ be their Lie algebras. Since the bracket of $\mathfrak{g}_{o}$ respects the Hodge filtration, $F^{0} \mathfrak{g}_{o}$ is a subalgebra of $\mathfrak{g}_{o}$. Denote the corresponding subgroup of $\mathcal{G}_{o}$ by $F^{0} \mathcal{G}_{o}$.

Let

$$
\Omega \in F^{0} W_{-1} K^{1}\left(\bar{X}_{\Gamma}, D ; \mathfrak{u}\right)
$$

be as above. Trivialize the pullback of $\boldsymbol{u}$ to $\mathfrak{h}$ using the sections $\left\{\mathbf{e}_{0}^{j} \cdot \mathbf{e}_{f}^{\prime}, \mathbf{e}_{0}^{j} \cdot \mathbf{e}_{f}^{\prime \prime}, \mathbf{e}_{0}^{j}\right.$. $\left.\mathbf{e}_{2 n}\right\}$ :

$$
\mathfrak{u} \times \mathfrak{h} \rightarrow \mathfrak{h} .
$$

This trivializes both the Hodge and weight filtrations. It also fixes an isomorphism $\mathcal{G}_{x} \cong \operatorname{SL}\left(H_{x}\right) \ltimes \mathcal{U}_{x}$ for all $x \in X_{\Gamma}$. Denote the pullback of $\Omega$ to $\mathfrak{h}$ by

$$
\widetilde{\Omega} \in\left(E^{1}(\mathfrak{h}) \hat{\otimes} \mathfrak{u}\right)^{\Gamma} .
$$


For convenience we now take the base point $\tau_{o}$ to be $i$. Since $\widetilde{\Omega}$ is integrable, the function $F: \mathfrak{h} \rightarrow \mathcal{U}_{o} \times \operatorname{SL}\left(H_{o}\right) \cong \mathcal{G}_{o}$ defined by

$$
\widetilde{F}(\tau):=\left(1+\int_{\tau_{o}}^{\tau} \widetilde{\Omega}+\int_{\tau_{o}}^{\tau} \widetilde{\Omega} \widetilde{\Omega}+\int_{\tau_{o}}^{\tau} \widetilde{\Omega} \widetilde{\Omega} \widetilde{\Omega}+\cdots,\left(\begin{array}{ll}
v & u / v \\
0 & v^{-1}
\end{array}\right)\right)
$$

where $\tau=u+i v^{2}$ (u,v real), is well defined and smooth. It induces a function $F: \mathfrak{h} \rightarrow \mathcal{G}_{o} / F^{0} \mathcal{G}_{o}$ which is equivariant with respect to the natural left $\Gamma$-actions on each.

Proposition 13.1. The function $F: \mathfrak{h} \rightarrow \mathcal{G}_{o} / F^{0} \mathcal{G}_{o}$ is holomorphic. (That is, it is an inverse limit of holomorphic functions.)

Proof. Set $H=H_{o}$. The map $\mathrm{SL}\left(H_{\mathbb{R}}\right) \rightarrow \mathfrak{h}$ that takes $g$ to $g \tau_{o}=g i$ induces an isomorphism $\mathrm{SL}\left(H_{\mathbb{R}}\right) / \mathrm{SO}(2) \rightarrow \mathfrak{h}$. The inclusion $\mathrm{SL}\left(H_{\mathbb{R}}\right) \hookrightarrow \mathrm{SL}\left(H_{\mathbb{C}}\right)$ induces an inclusion

$$
\mathfrak{h}=\mathrm{SL}\left(H_{\mathbb{R}}\right) / \mathrm{SO}(2) \hookrightarrow \mathrm{SL}\left(H_{\mathbb{C}}\right) / F^{0} \cong \mathbb{P}^{1} .
$$

Since the right hand matrix in $\widetilde{F}$ is in $\operatorname{SL}\left(H_{\mathbb{R}}\right)$ and takes $i$ to $\tau$, the composition of $F$ with the projection $\mathcal{G}_{o} / F^{0} \mathcal{G}_{o} \rightarrow \operatorname{SL}\left(H_{\mathbb{C}}\right) / F^{0}$ is the inclusion $\mathfrak{h} \hookrightarrow \operatorname{SL}\left(H_{\mathbb{C}}\right) / F^{0}$. Consequently, to show that $F$ is holomorphic, we need only check that its first factor is holomorphic. Write $\widetilde{\Omega}=\widetilde{\Omega}^{\prime}+\widetilde{\Omega}^{\prime \prime}$, where $\widetilde{\Omega}^{\prime}$ has type $(1,0)$ and $\widetilde{\Omega}^{\prime \prime}$ has type $(0,1)$. Since $\Omega \in F^{0} K^{1}\left(\bar{X}_{\Gamma}, D ; \mathfrak{u}\right)$,

$$
\widetilde{\Omega}^{\prime \prime} \in E^{0,1}(\mathfrak{h}) \hat{\otimes} F^{0} \mathfrak{u} .
$$

The fundamental theorem of calculus implies that $F$ satisfies the differential equation $d \widetilde{F}=\widetilde{F} \widetilde{\Omega}$ from which it follows that $\bar{\partial} \widetilde{F}=\widetilde{F} \Omega^{\prime \prime}$. This implies the vanishing of $\bar{\partial} F$.

The projection $\mathcal{G}_{o} \rightarrow \mathrm{SL}\left(H_{o, \mathbb{C}}\right)$ preserves the Hodge filtration and induces a holomorphic, $\Gamma$-invariant projection $\mathcal{G}_{o} / F^{0} \mathcal{G}_{o} \rightarrow \mathrm{SL}\left(H_{o}\right) / F^{0} \cong \mathbb{P}^{1}$. Then one has the diagram

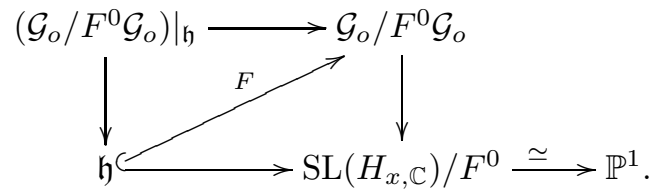

Recall that our choices have fixed an isomorphism $\mathcal{G}_{o} \cong \mathrm{SL}\left(H_{o}\right) \ltimes \mathcal{U}_{o}$. The natural homomorphism corresponds to a non-abelian 1-cocycle $\Theta_{o}: \Gamma \rightarrow \mathcal{U}_{o}(\mathbb{Q})$.

Lemma 13.2. The function $F: \mathfrak{h} \rightarrow \mathcal{G}_{o} / F^{0}$ satisfies $F(\gamma \tau)=\Theta_{o}(\gamma) F(\gamma \tau)$ for all $\gamma \in \Gamma$.

Proof. The identification $\mathcal{G}_{o} \cong \mathrm{SL}\left(H_{o}\right) \ltimes \mathcal{U}_{o}$ induces an identification of $\mathcal{G}_{o} / F^{0}$ with $\mathcal{U}_{o} \times \mathbb{P}^{1}$. If $c_{\gamma}$ is a path from $\tau_{o}$ to $\gamma \tau_{o}$ and $c$ is a path from $\tau_{o}$ to $\tau$, then $c_{\gamma} *\left(\gamma \cdot c_{\gamma}\right)$ is a path from $\tau_{o}$ to $\gamma \tau$. So

$$
F(\gamma \tau)=\left(\Theta_{o}(\gamma) T(c)^{-1}, \gamma \tau\right)=\Theta_{o}(\gamma) F(\tau)
$$

where $T^{-1}$ denotes $1+\int \widetilde{\Omega}+\int \widetilde{\Omega} \widetilde{\Omega}+\cdots$.

Let $\mathcal{U}_{o, \mathbb{Z}}$ be the subgroup of $\mathcal{U}_{o}$ that is generated by $\left\{\Theta_{o}(\gamma): \gamma \in \Gamma\right\}$. Let $\mathcal{G}_{o, \mathbb{Z}}$ be the subgroup of $\mathcal{G}_{o} \cong \mathrm{SL}\left(H_{o}\right) \cong \mathcal{U}_{o}$ that corresponds to $\operatorname{SL}\left(H_{o, \mathbb{Z}}\right) \ltimes \mathcal{U}_{o, \mathbb{Z}}$. The previous result implies that $F(\gamma \tau)$ and $\gamma F(\tau)$ lies in the same left $\mathcal{U}_{o, \mathbb{Z}}$ orbit. 
The universal relative albanese manifold $\mathcal{A}_{\Gamma}$ of $X_{\Gamma}$ is defined by

$$
\mathcal{A}_{\Gamma}=\mathcal{U}_{o, \mathbb{Z}} \backslash\left(\left.\left(\mathcal{G}_{o} / F^{0} \mathcal{G}_{o}\right)\right|_{\mathfrak{h}}\right) .
$$

There is a natural quotient mapping to $X_{\Gamma}$. Taking the quotient of the left-hand map of the previous diagram by $\Gamma$ gives the universal non-abelian Albanese map

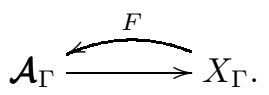

The fiber over $x_{o} \in X_{\Gamma}$ is isomorphic to $\mathcal{U}_{o, \mathbb{Z}} \backslash \mathcal{U}_{o} / F^{0}$.

Remark 13.3. For each finite dimensional quotient $G_{\alpha}$ of $\mathcal{G}_{o}$ in the category of groups with a MHS, one can define

$$
\mathcal{A}_{\alpha}=G_{\alpha, \mathbb{Z}} \backslash\left(\left.\left(G_{\alpha} / F^{0} G_{\alpha}\right)\right|_{\mathfrak{h}}\right) .
$$

Since $\mathcal{G}_{o}$ is the inverse limit of the $G_{\alpha}, \mathcal{A}_{\Gamma}=\lim \mathcal{A}_{\alpha}$. The reduction of the section $F$ is a holomorphic section $F_{\alpha}$ of this bundle. In particular, when $\Gamma$ is a congruence subgroup, by taking $G_{\alpha}$ to be the quotient

$$
0 \rightarrow \check{M}_{f} \otimes S^{n} H_{o} \rightarrow G_{n} \rightarrow \mathrm{SL}\left(H_{o}\right) \rightarrow 1
$$

of $\mathcal{G}_{o}$, where $M_{f}$ is the smallest $\mathbb{Q}$-sub HS of $H^{1}\left(X_{\Gamma}, S^{m} \mathbb{H}\right)$ that contains $\omega_{f}(\mathbf{e})$ and $\check{M}_{f}$ its dual, we see that for each $f \in \mathfrak{B}_{n+2}(\Gamma)$, there is a bundle over $X_{\Gamma}$ with fiber over $x \in X_{\Gamma}$ the intermediate jacobian

$$
\operatorname{Ext}_{\mathrm{MHS}}^{1}\left(\mathbb{Z}, \check{M}_{f, \mathbb{Z}} \otimes S^{n} H_{x, \mathbb{Z}}\right)
$$

with a holomorphic section induced by $F$. We will see below that this is a normal function which is the period mapping of an extension of $\mathbb{Q}$ by $\check{M}_{f} \otimes S^{n} \mathbb{H}$. When $\Gamma$ is a congruence subgroup, each Eisenstein series determines a normal function that corresponds to an extension of $\mathbb{Z}$ by $S^{n} \mathbb{H}(n+1)$.

The coefficients of $F$ are holomorphic functions on $\mathfrak{h}$ which can be realized as periods of admissible variations of MHS. These include iterated integrals of (holomorphic) modular forms, but there are many more. Below is an example of such a holomorphic iterated integral that is not of the type considered by Manin. It is, in some sense, a generalization of the Riemann theta function. (Cf. [17, Ex. 4.4].)

Example 13.4. Suppose that $f \in M_{2 n+2}^{o}$ and $g \in M_{2 m+2}^{o}$ are cusp forms, not necessarily of the same weight. Since $\mathrm{SL}_{2}(\mathbb{Z})$ has cohomological dimension 1 , the form $\bar{\omega}_{f}\left(\mathbf{e}_{f}^{\prime \prime}\right) \wedge \omega_{g}\left(\mathbf{e}_{g}^{\prime}\right)$ is exact. Lemma 6.1 implies that there is

$$
\xi \in F^{0} W_{-1} K^{1}\left(\bar{X}_{\Gamma}, D ; \mathbb{S}^{2 n}\left(\mathbf{e}_{f}^{\prime \prime}\right) \otimes \mathbb{S}^{m}\left(\mathbf{e}_{g}^{\prime}\right)\right)
$$

such that

$$
\bar{\partial} \xi+\bar{\omega}_{f}\left(\mathbf{e}_{f}^{\prime \prime}\right) \wedge \omega_{g}\left(\mathbf{e}_{g}^{\prime}\right)=0 .
$$

Then

$$
\tau \mapsto \int_{\tau_{o}}^{\tau} \bar{\omega}_{f}\left(\mathbf{e}_{f}^{\prime \prime}\right) \omega_{g}\left(\mathbf{e}_{g}^{\prime}\right)+\xi
$$

is a well defined function from $\mathfrak{h}$ to $S^{2 n} H_{o} \otimes S^{2 m} H_{o}$. An elementary argument (cf. [17, Prop. 4.3]) implies that it is holomorphic.

Perhaps the most interesting version of this example is where $f=g$ and one composes it with an invariant bilinear form $S^{2 n} H_{o} \otimes S^{2 n} H_{o} \rightarrow \mathbb{C}$. Such iterated integrals occur as periods of biextensions. 
13.2. Iterated Shimura integrals. In [29, 30] Manin considered iterated integrals of holomorphic modular forms and non-abelian generalizations of modular symbols. Here we briefly discuss the relationship of his work to the Hodge theory of modular groups. Recall that $\mathfrak{B}_{w}=\mathfrak{B}_{w}\left(\mathrm{SL}_{2}(\mathbb{Z})\right)$.

Manin considers iterated integrals with values in the algebras

$$
A=\mathbb{C}\left\langle\left\langle\mathbf{e}_{0}^{j} \cdot \mathbf{e}_{2 n}, \mathbf{e}_{0}^{j} \cdot \mathbf{e}_{f}^{\prime}: f \in \mathfrak{B}_{2 n}, 0 \leq j \leq 2 n-2, n \geq 2\right\rangle\right\rangle
$$

and

$$
B=\mathbb{C}\left\langle\left\langle\mathbf{e}_{0}^{j} \cdot \mathbf{e}_{2 n}: 0 \leq j \leq 2 n-2, n \geq 2\right\rangle\right\rangle .
$$

These iterated integrals are of the form

$$
1+\int_{\tau_{o}}^{\tau} \Omega+\int_{\tau_{o}}^{\tau} \Omega \Omega+\int_{\tau_{o}}^{\tau} \Omega \Omega \Omega+\cdots
$$

where

$$
\Omega=\Omega_{A}:=\sum_{n \geq 2}\left(\psi_{2 n}\left(\mathbf{e}_{2 n}\right)+\sum_{f \in \mathfrak{B}_{2 n}} \omega_{f}\left(\mathbf{e}_{f}^{\prime}\right)\right)
$$

in the first case and

$$
\Omega=\Omega_{B}:=\sum_{n \geq 2} \psi_{2 n}\left(\mathbf{e}_{2 n}\right)
$$

in the second case. Both of these forms are $\Gamma$-invariant.

Let $\mathfrak{u}_{A}\left(\right.$ resp. $\left.\mathfrak{u}_{B}\right)$ be the set of primitive elements of $A$ (resp. $\left.\mathfrak{u}_{B}\right)$. Then $\Omega_{A}$ $\left(\right.$ resp. $\left.\Omega_{B}\right)$ takes values in $\mathfrak{u}_{A}\left(\right.$ resp. $\left.\mathfrak{u}_{B}\right)$. Set

$$
\mathfrak{u}=\mathbb{L}\left(\mathbf{e}_{0}^{j} \cdot \mathbf{e}_{2 n}, \mathbf{e}_{0}^{j} \cdot \mathbf{e}_{f}^{\prime}, \mathbf{e}^{0} \cdot \mathbf{e}_{f}^{\prime \prime}: f \in \mathfrak{B}_{2 n}, 0 \leq j \leq 2 n-2, n \geq 2\right)^{\wedge} .
$$

It follows from Figure 5 that $F^{0} \mathfrak{u}$ is generated by $\left\{\mathbf{e}_{0}^{j} \cdot \mathbf{e}_{f}^{\prime \prime}: f \in \mathfrak{B}_{2 n}, n \geq 2\right\}$, so that $\mathfrak{u}_{A}$ is the quotient of $\mathfrak{u}$ by the ideal $\left(F^{0} \mathfrak{u}\right)$ generated by $F^{0} \mathfrak{u}$, and $\mathfrak{u}_{B}$ is the quotient of $\mathfrak{u}$ by the ideal generated by all $\mathbf{e}_{0}^{j} \cdot \mathbf{e}_{f}^{\prime}$ and $\mathbf{e}_{0} \cdot \mathbf{e}_{f}^{\prime \prime}$. His iterated integral is the reduction of the one in the previous section (the first argument of $\widetilde{F}$ ) $\bmod$ these ideals. This implies that there are many interesting holomorphic iterated integrals which do not occur as iterated Shimura integrals.

One can ask whether the $\mathrm{SL}_{2}(\mathbb{Z})$ connection on the local system

$$
\mathfrak{u}_{B} \times \mathfrak{h} \rightarrow \mathfrak{h}
$$

defined by $\Omega_{B}$ descends to an admissible VMHS over $\mathcal{M}_{1,1}$. It will follow from Theorem 19.4 that it does not as we explain in Remark 19.5.

13.3. Extensions of variations of MHS associated to Eisenstein series. Here we suppose, for simplicity, that $\Gamma=\mathrm{SL}_{2}(\mathbb{Z})$. In this section, we sketch an explicit construction of an extension

$$
0 \rightarrow S^{2 n} \mathbb{H}(2 n+1) \rightarrow \mathbb{E} \rightarrow \mathbb{Q} \rightarrow 0
$$

for each Eisenstein series $G_{2 n+2} \cdot{ }^{19}$

Let $H=\mathbb{C a} \oplus \mathbb{C}$. Define the Hodge filtration on $H$ by $F^{0} H=H$ and $F^{1} H=$ $\mathbb{C w}$. This induces a Hodge filtration on $S^{2 n} H$. Trivialize the bundle $\mathcal{H}_{\mathfrak{h}} \rightarrow \mathfrak{h}$ with the sections a and $\mathbf{w}$ :

$$
\mathcal{H}_{\mathfrak{h}} \cong H \times \mathfrak{h}
$$

\footnotetext{
${ }^{19}$ This construction works equally well when $\Gamma$ is a congruence subgroup. The construction in the more general case is sketched at the end of the next section.
} 
Trivialize $S^{2 n} \mathcal{H}_{\mathfrak{h}}$ using monomials in a and $\mathbf{w}$. Then $F^{p} S^{2 n} \mathcal{H}_{\mathfrak{h}}$ is trivialized by the sections $\left\{\mathbf{a}^{2 n-j} \mathbf{w}^{j}: j \geq p\right\}$.

Set $V=\mathbb{C} e \oplus S^{2 n} H(2 n+1)$ and $\mathcal{V}_{\mathfrak{h}}=V \times \mathfrak{h}$. Define Hodge and weight filtrations on $V$ by giving e type $(0,0)$ and $\mathbf{a}^{2 n-j} \mathbf{w}^{j}$ type $(j-2 n-1,-j-1)$. Let $\mathrm{SL}_{2}(\mathbb{Z})$ act in this bundle by acting trivially on $\mathbf{e}$, and on $\mathbf{a}$ and $\mathbf{w}$ by the factor of automorphy given in Corollary 9.4. The Hodge and weight filtrations are invariant under this action, so that they descend to Hodge and weight filtrations on the (orbifold) quotient bundle

$$
\mathcal{V}:=\mathrm{SL}_{2}(\mathbb{Z}) \backslash \mathcal{V}_{\mathfrak{h}} \rightarrow \mathcal{M}_{1,1}
$$

This bundle is trivial over the punctured $q$-disk $\Delta^{*}$. Extend it to a bundle $\overline{\mathcal{V}}$ over $\overline{\mathcal{M}}_{1,1}$ by defining its sections over the $q$-disk $\Delta$ to be $V \otimes \mathcal{O}_{\Delta}$. The Hodge and weight bundles clearly extend to sub-bundles of $\overline{\mathcal{V}}$.

Define a connection on $\mathcal{V}_{\mathfrak{h}}$ by $d+\Omega$, where

$$
\Omega=\left(\begin{array}{cc}
0 & 0 \\
\psi_{2 n+2}\left(\mathbf{w}^{2 n}\right) & \mathbf{a} \frac{\partial}{\partial \mathbf{w}} \frac{d q}{q}
\end{array}\right) \in\left(\begin{array}{cc}
\mathbb{C} & 0 \\
S^{2 n} H & \text { End } S^{2 n} H
\end{array}\right) \frac{d q}{q} .
$$

It is holomorphic, flat and $\Gamma$-invariant. It therefore descends to a flat connection $\nabla$ on $\mathcal{V}$ which has a regular singular point at the cusp when viewed as a connection on $\overline{\mathcal{V}}$. This implies that the extended bundle is Deligne's canonical extension of $(\mathcal{V}, \nabla)$ to $\overline{\mathcal{M}}_{1,1}$. Since

$$
\Omega \in\left(F^{-1} W_{-1} \text { End } V\right) \otimes \mathcal{O}(\Delta) \frac{d q}{q},
$$

the weight filtration is flat and the connection satisfies Griffiths transversality. Since $\psi_{2 n+2}\left(\mathbf{w}^{2 n}\right)$ has rational periods, it follows that the local system $\mathbb{V}$ associated to $(\mathcal{V}, \nabla)$ has a natural $\mathbb{Q}$-form. The associated weight graded local system is

$$
\mathrm{Gr}_{\bullet}^{W} \mathbb{V}=\mathbb{Q}(0) \oplus S^{2 n} \mathbb{H}(2 n+1) .
$$

The existence of a relative weight filtration at $q=0$ follows from the argument in the proof of Theorem 7.17 It follows that $\mathbb{V}$ is an admissible variation of MHS over $\mathcal{M}_{1,1}$. The results of Section 18.4 imply that every extension of $\mathbb{Q}$ by $S^{2 n} \mathbb{H}(m)$ over $\mathcal{M}_{1,1}$ is a multiple of this extension when $m=2 n+1$ and trivial otherwise.

13.4. Extensions of variations of MHS associated to cusp forms. The construction of the extension corresponding to an eigen cusp form is similar, but a little more elaborate. Suppose that $\Gamma$ is a congruence subgroup of $\mathrm{SL}_{2}(\mathbb{Z})$ and that $m \geq 0$. The first step is to construct an extension

$$
0 \rightarrow H^{1}\left(X_{\Gamma}, S^{m} \mathbb{H}_{\mathbb{Z}}\right)^{*} \otimes S^{m} \mathbb{H}_{\mathbb{Z}} \rightarrow \mathbb{V} \rightarrow \mathbb{Z} \rightarrow 0
$$

in the category of $\mathbb{Z}$-MHS over $X_{\Gamma}$.

Denote the completion of $X_{\Gamma}$ by $\bar{X}_{\Gamma}$. Let $\overline{\mathcal{V}}$ be the $C^{\infty}$ vector bundle over $\bar{X}_{\Gamma}$ associated to the canonical extension

$$
\mathcal{O}_{\bar{X}_{\Gamma}} \oplus H^{1}\left(X_{\Gamma}, S^{m} \mathbb{H}\right)^{*} \otimes S^{m} \overline{\mathcal{H}}
$$

of the admissible variation $\mathbb{Q} \oplus H^{1}\left(X_{\Gamma}, S^{m} \mathbb{H}\right)^{*} \otimes S^{m} \mathbb{H}$ over $X_{\Gamma}$. This has natural Hodge and weight sub-bundles. Denote the restriction of $\overline{\mathcal{V}}$ to $X_{\Gamma}$ by $\mathcal{V}$ and the direct sum connection on it by $\nabla_{0}$.

Define a $C^{\infty}$ connection on $\mathcal{V}$ by $\nabla=\nabla_{0}+\Omega_{1, m}$, where $\Omega_{1, m}$ is the form defined in Equation (12.4). This connection is flat, and thus defines a new holomorphic structure on the bundle $\mathcal{V}$. Arguments almost identical to those in Section 7.4 show 
that $(\overline{\mathcal{V}}, \nabla)$ is Deligne's canonical extension of $(\mathcal{V}, \nabla)$, that the Hodge bundles are holomorphic sub-bundles of $\overline{\mathcal{V}}$ with respect to this new complex structure, and that the connection $\nabla$ satisfies Griffiths transversality. The existence of a relative weight filtration at each cusp is established as in the proof of Theorem 7.17. The fact that $\Omega_{1, m}$ represents the identity $H^{1}\left(X_{\Gamma}, S^{m} \mathbb{H}\right) \rightarrow H^{1}\left(X_{\Gamma}, S^{m} \mathbb{H}\right)$ implies that the local system $\mathbb{V}$ underlying the flat bundle $(\mathcal{V}, \nabla)$ has a natural $\mathbb{Z}$-form. It follows that there is an admissible $\mathbb{Z}$-VMHS $\mathbb{V}$ over $X_{\Gamma}$ whose corresponding $C^{\infty}$ vector bundle is $\mathcal{V}$ and whose weight graded quotients are $\mathbb{Q}(0)$ and $H^{1}\left(X_{\Gamma}, S^{m} \mathbb{H}_{\mathbb{Z}}\right)^{*} \otimes S^{m} \mathbb{H}$. (Cf. Lemma 7.9.)

Having constructed the extension (13.6), we can now construct the extension corresponding to a Hecke eigen cusp form $f \in \mathfrak{B}_{m+2}(\Gamma)$. The smallest sub $\mathbb{Q}$ HS $M_{f}$ of $H^{1}\left(X_{\Gamma}, S^{m} \mathbb{H}_{\mathbb{Q}}\right)$ whose complexification contains $\omega_{f}(\mathbf{e})$ is pure of weight $m+1$. So $\check{M}_{f} \otimes S^{m} \mathbb{H}$ is pure of weight -1 . The corresponding extension

$$
0 \rightarrow \check{M}_{f} \otimes S^{m} \mathbb{H}_{\mathbb{Z}} \rightarrow \mathbb{E}_{f} \rightarrow \mathbb{Q} \rightarrow 0
$$

is obtained by pushing out the extension (13.6) along the dual of the inclusion

$$
M_{f} \hookrightarrow H^{1}\left(X_{\Gamma}, S^{m} \mathbb{H}_{\mathbb{Z}}\right) .
$$

This extension has a natural $\mathbb{Z}$-form, which we denote by $E_{f, \mathbb{Z}}$.

The extension $\mathbb{E}_{f, \mathbb{Z}}$ corresponds to a holomorphic section of the associated bundle of intermediate jacobians, which has fiber

$$
J\left(H^{1}\left(X_{\Gamma}, S^{m} \mathbb{H}\right)^{*} \otimes S^{m} H_{x}\right)
$$

over $x \in X_{\Gamma}$, where for a $\mathbb{Z}$-MHS $V$ with negative weights

$$
J(V):=V_{\mathbb{C}} /\left(V_{\mathbb{Z}}+F^{0} V_{\mathbb{C}}\right) \cong \operatorname{Ext}_{\mathrm{MHS}}^{1}(\mathbb{Z}, V) .
$$

The section is obtained by integrating the invariant 1 -form $\omega_{f}\left(\mathbf{e}_{f}^{\prime}\right)+\omega_{f}\left(\mathbf{e}_{f}^{\prime \prime}\right)$. More sections can be obtained by applying elements of Aut $M_{f, \mathbb{Z}}$.

A similar construction can be used to construct the extension of a normalized Eisenstein series $f$. When $\Gamma=\mathrm{SL}_{2}(\mathbb{Z})$ this reduces to the construction in the previous section. In this case, the smallest $\mathbb{Q}$-Hodge sub structure $M_{f}$ of $H^{1}\left(X_{\Gamma}, S^{m} \mathbb{H}\right)$ that contains $\psi_{f}(\mathbf{e})$ is $M_{f}=\mathbb{Q}(-m-1)$. Pushing out the extension (13.6) along the inclusion $M_{f} \rightarrow H^{1}\left(X_{\Gamma}, S^{m} \mathbb{H}\right)$ gives the extension

$$
0 \rightarrow S^{m} \mathbb{H}(m+1) \rightarrow \mathbb{E}_{f} \rightarrow \mathbb{Q} \rightarrow 0 .
$$

corresponding to $f$.

\section{The Relative Completion of $\pi_{1}\left(\mathcal{M}_{1, \overrightarrow{1}}, x\right)$}

By a sleight of hand, can deduce the MHS on the unipotent radical of the relative completion of the fundamental group of $\mathcal{M}_{1, \overrightarrow{1}}$ from the MHS on the unipotent radical of the relative completion of $\mathrm{SL}_{2}(\mathbb{Z})$. The MHS on this completion is of interest as it acts on the unipotent completion of the fundamental group of a once punctured elliptic curve.

First recall some classical facts. (Detailed proofs can be found, for example, in [19].) The moduli space $\mathcal{M}_{1, \overrightarrow{1}}$ of elliptic curves with a non-zero tangent vector at the identity is the complement of the discriminant locus $u^{3}-27 v^{2}=0$ in $\mathbb{C}^{2}$. For us, it is more useful to write it as the quotient of $\mathbb{C}^{*} \times \mathfrak{h}$ by the action

$$
\gamma:(\xi, \tau) \mapsto\left((c \tau+d)^{-1} \xi, \gamma \tau\right)
$$


where $\gamma=\left(\begin{array}{ll}a & b \\ c & d\end{array}\right)$. This action is fixed point free, so that $\mathcal{M}_{1, \overrightarrow{1}}$ is an analytic variety. The projection $\mathbb{C} \times \mathfrak{h} \rightarrow \mathfrak{h}$ induces a projection $\pi: \mathcal{M}_{1, \overrightarrow{1}} \rightarrow \mathcal{M}_{1,1}$ that is the $\mathbb{C}^{*}$ bundle associated to the orbifold line bundle $\mathcal{L} \rightarrow \mathcal{M}_{1,1}$ with factor of automorphy $c \tau+d$. Modular forms of $\mathrm{SL}_{2}(\mathbb{Z})$ of weight $m$ are sections of $\mathcal{L}^{\otimes m}$. (Cf. [19, §4].) The cusp form $\Delta$ of $\mathrm{SL}_{2}(\mathbb{Z})$ of weight 12 trivializes $\mathcal{L}^{\otimes 12}$.

The $\mathrm{SL}_{2}(\mathbb{Z})$ action lifts to an action of a central extension

$$
0 \rightarrow \mathbb{Z} \rightarrow \widehat{\Gamma} \rightarrow \mathrm{SL}_{2}(\mathbb{Z}) \rightarrow 1
$$

on $\mathbb{C} \times \mathfrak{h} .{ }^{20}$ The group $\widehat{\Gamma}$ is the mapping class group of a genus 1 surface with one boundary component. ${ }^{21}$ This extension corresponds to the orbifold $\mathbb{C}^{*}$-bundle $\mathcal{M}_{1, \overrightarrow{1}} \rightarrow \mathcal{M}_{1,1}$.

Denote the completion of $\widehat{\Gamma}$ with respect to the homomorphism

$$
\widehat{\Gamma} \rightarrow \mathrm{SL}_{2}(\mathbb{Z}) \hookrightarrow \mathrm{SL}_{2}(\mathbb{Q})
$$

by $\widehat{\mathcal{G}}$ and its prounipotent radical by $\widehat{\mathcal{U}}$. Denote the completion of $\mathrm{SL}_{2}(\mathbb{Z})$ with respect to its inclusion into $\mathrm{SL}_{2}(\mathbb{Q})$ by $\mathcal{G}$ and its prounipotent radical by $\mathcal{U}$. Denote the Lie algebras of $\mathcal{U}$ and $\widehat{\mathcal{U}}$ by $\mathfrak{u}$ and $\widehat{\mathfrak{u}}$, respectively. The projection $\widehat{\Gamma} \rightarrow \mathrm{SL}_{2}(\mathbb{Z})$ induces a homomorphism $\widehat{\mathcal{G}} \rightarrow \mathcal{G}$ that commutes with the projections to $\mathrm{SL}_{2}$.

Proposition 14.2. For each choice of a base point $x \in \mathcal{M}_{1,1}$ and each lift $\widehat{x}$ of $x$ to $\mathcal{M}_{1, \overrightarrow{1}}$, there is a natural isomorphism

$$
\widehat{\mathcal{G}_{\widehat{x}}} \cong \mathcal{G}_{x} \times \mathbb{G}_{a}(1),
$$

where $\mathbb{G}_{a}(1)$ denotes the copy of $\mathbb{G}_{a}$ with the $M H S \mathbb{Q}(1)$. This induces an isomorphism of $M H S$

$$
\widehat{\mathfrak{g}}_{\widehat{x}} \cong \mathfrak{g}_{x} \oplus \mathbb{Q}(1) .
$$

where $\widehat{\mathfrak{g}}_{\widehat{x}}$ is given the natural MHS constructed in $[15]$.

Proof. Since the weight 12 cusp form $\Delta$ trivializes $\mathcal{L}^{\otimes 12}$ and since $\mathcal{M}_{1, \overrightarrow{1}}$ is $\mathcal{L}^{*}$, there is a 12 -fold covering

$$
\mathcal{M}_{1, \overrightarrow{1}} \rightarrow \mathcal{M}_{1,1} \times \mathbb{C}^{*}
$$

that commutes with the projections to $\mathcal{M}_{1,1}$. It induces an inclusion

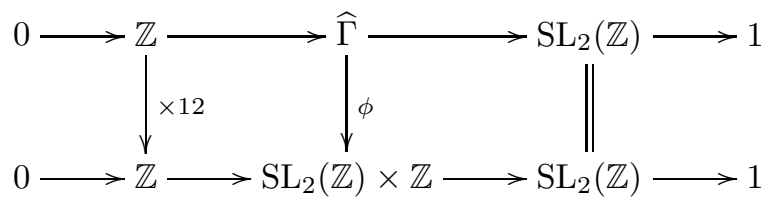

of extensions. The completion of $\mathrm{SL}_{2}(\mathbb{Z}) \times \mathbb{Z}$ with respect to the obvious homomorphism to $\mathrm{SL}_{2}(\mathbb{Q})$ is $\mathcal{G} \times \mathbb{G}_{a}$. This and the right exactness (Prop. 3.6) of relative

\footnotetext{
${ }^{20}$ This action can be understood as follows: The quotient of $\mathbb{C} \times \mathfrak{h}$ by the central $\mathbb{Z}$ in $\widehat{\Gamma}$ is $\mathbb{C}^{*} \times \mathfrak{h}$. The quotient mapping is the exponential mapping on the first factor; $\mathrm{SL}_{2}(\mathbb{Z})$ acts on $\mathbb{C}^{*} \times \mathfrak{h}$ with factor of automorphy $(c \tau+d)^{2}$.

${ }^{21}$ The group $\widehat{\Gamma}$ is isomorphic to the 3 -string braid group $B_{3}$ and also to the inverse image of $\mathrm{SL}_{2}(\mathbb{Z})$ in the universal covering group of $\mathrm{SL}_{2}(\mathbb{R})$. (Cf. $[19, \S 8]$.)
} 
completion imply that the commutative diagram

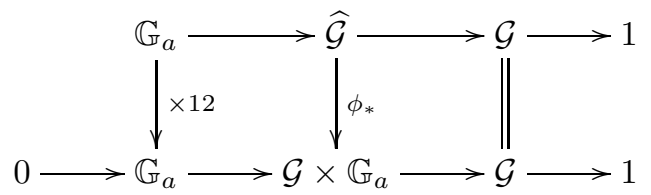

has exact rows. It follows that $\phi_{*}: \widehat{\mathcal{G}} \rightarrow \mathcal{G} \times \mathbb{G}_{a}$ is an isomorphism. The Hodge theoretic statements follow from the functoriality of the MHS on relative completion.

There is therefore an isomorphism $\widehat{\mathfrak{u}} \cong \mathfrak{u} \oplus \mathbb{C e}_{2}$, in the category of pronilpotent Lie algebras with an $\mathrm{SL}_{2}$ action. The new generator $\mathbf{e}_{2}$ spans a copy of the trivial representation of $\mathrm{SL}_{2}$ and commutes with the remaining generators

$$
\bigcup_{n>0}\left\{\mathbf{e}_{0}^{j} \cdot \mathbf{e}_{f}^{\prime}, \mathbf{e}_{0}^{j} \cdot \mathbf{e}_{f}^{\prime \prime}, \mathbf{e}_{0}^{j} \cdot \mathbf{e}_{2 n}: 0 \leq j \leq 2 n-2, f \in \mathfrak{B}_{2 n}\right\} .
$$

The Hodge type of $\mathbf{e}_{2}$ is $(-1,-1)$, which is consistent with the Hodge types of the other $\mathbf{e}_{2 m+2, P}$ given in Figure 4 .

Remark 14.3. One can lift the power series connection $\Omega$ whose monodromy representation $\mathrm{SL}_{2}(\mathbb{Z}) \rightarrow \mathrm{SL}_{2} \ltimes \mathcal{U}$ is the relative completion of $\mathrm{SL}_{2}(\mathbb{Z})$ to a power series connection $\widehat{\Omega}$ whose monodromy homomorphism $\widehat{\Gamma} \rightarrow \mathrm{SL}_{2} \ltimes \widehat{\mathcal{U}}$ is the relative completion of $\widehat{\Gamma}$.

The normalized Eisenstein series $G_{2}(\tau)$ is also defined by the series (12.6), suitably summed. Although $G_{2}$ is not a modular form, it satisfies (cf. [38, pp. 95-96])

$$
G_{2}(\gamma \tau)=(c \tau+d)^{2} G_{2}(\tau)+i c(c \tau+d) / 4 \pi
$$

This implies that

$$
\psi_{2}:=2 \pi i G_{2}(\tau) d \tau-\frac{1}{2} \frac{d \xi}{\xi} \in E^{1}\left(\mathbb{C}^{*} \times \mathfrak{h}\right)
$$

is $\mathrm{SL}_{2}(\mathbb{Z})$-invariant, and thus a closed 1 -form on $\mathcal{M}_{1, \overrightarrow{1}} \cdot{ }^{22}$

If $\Omega \in K^{1}\left(\overline{\mathcal{M}}_{1,1}, P ; \mathfrak{u}\right)$ is a power series connection (as constructed above), then

$$
\widehat{\Omega}=\Omega+\psi_{2} \mathbf{e}_{2}
$$

is an integrable $\mathrm{SL}_{2}(\mathbb{Z})$-invariant power series connection with values in $\widehat{\mathfrak{u}}:=\mathfrak{u} \oplus \mathbb{C e}_{2}$. For each choice of a lift $\widehat{x} \in \mathbb{C} \times \mathfrak{h}$ of a base point $x \in \mathcal{M}_{1, \overrightarrow{1}}$, the monodromy representation

$$
\widehat{\Gamma} \rightarrow \mathrm{SL}_{2}(\mathbb{C}) \ltimes \widehat{\mathcal{U}}
$$

induces isomorphisms $\widehat{\mathcal{G}}_{x} \cong \mathrm{SL}_{2} \ltimes \widehat{\mathcal{U}} \cong\left(\mathrm{SL}_{2} \ltimes \mathcal{U}\right) \times \mathbb{G}_{a}$.

\footnotetext{
${ }^{22}$ It is useful to note that $\psi_{2}=-\frac{1}{24} \frac{d D}{D}$, where, where $D=u^{3}-27 v^{2}$ denotes the discriminant function on $\mathcal{M}_{1, \overrightarrow{1}}$. This is because there is a unique logarithmic 1 -form on $\mathcal{M}_{1, \overrightarrow{1}}$ with given residue along the divisor of nodal cubics. Cf. [20, Eqn. 19.1], where $D$ is denoted $\Delta$.
} 


\section{The Monodromy Representation}

Let $E$ be an elliptic curve with identity 0 . Set $E^{\prime}=E-\{0\}$ and let $\vec{v} \in T_{0} E$ be a non-zero tangent vector. Denote the Lie algebra of the unipotent completion of $\pi_{1}\left(E^{\prime}, \vec{v}\right)$ by $\mathfrak{p}(E, \vec{v})$. Recall from Section 3.4.3 that this is a completed free Lie algebra with abelianization $H_{1}(E)$.

Denote by $\mathfrak{p}$ the local system over $\mathcal{M}_{1, \overrightarrow{1}}$ whose fiber over $[E, \vec{v}]$ by $\mathfrak{p}(E, \vec{v})$. Fix a base point $x_{o}=\left[E_{o}, \vec{v}_{o}\right]$ of $\mathcal{M}_{1, \overrightarrow{1}}$. Set $H_{o}=H_{1}\left(E_{o}\right)$ and $\mathfrak{p}_{o}=\mathfrak{p}\left(E_{o}, \vec{v}_{o}\right)$. Denote the completion of $\pi_{1}\left(\mathcal{M}_{1, \overrightarrow{1}}, x_{o}\right)$ relative to the standard homomorphism to $\operatorname{SL}\left(H_{o}\right)$ by $\widehat{\mathcal{G}}_{o}$ and its prounipotent radical by $\widehat{\mathcal{U}}_{o}$. Denote their Lie algebras by $\widehat{\mathfrak{g}}_{o}$ and $\widehat{\mathfrak{u}}_{o}$, respectively.

The monodromy action $\pi_{1}\left(\mathcal{M}_{1, \overrightarrow{1}}, x_{o}\right) \rightarrow$ Aut $\mathfrak{p}_{o}$ respects the lower central series of $\mathfrak{p}_{o}$ and acts on each graded quotient through an action of $\operatorname{SL}\left(H_{o}\right)$. The universal mapping property of relative completion implies that the monodromy representation above induces a homomorphism

$$
\widehat{\mathcal{G}}_{o} \rightarrow \text { Aut } \mathfrak{p}_{o} \text {. }
$$

This induces a homomorphism $\widehat{\mathfrak{g}}_{o} \rightarrow$ Der $\mathfrak{p}_{o}$ that we shall call the infinitesimal monodromy action.

Proposition 15.1. The infinitesimal monodromy action $\widehat{\mathfrak{g}}_{o} \rightarrow$ Der $\mathfrak{p}_{o}$ is a morphism of MHS.

Sketch of Proof. The universal punctured elliptic curve $\mathcal{E}^{\prime} \rightarrow \mathcal{M}_{1, \overrightarrow{1}}$ has fiber $E^{\prime}$ over $[E, \vec{v}] \in \mathcal{M}_{1, \overrightarrow{1}}$. The tangent vector $\vec{v}_{o}$ of $E$ at 0 can be regarded as a tangential base point of $\mathcal{E}^{\prime}$. The diagram

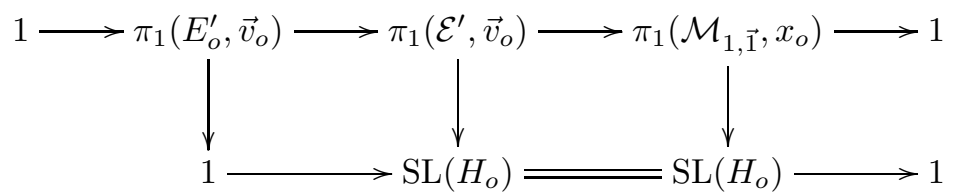

gives rise to an exact sequence

$$
1 \rightarrow \mathcal{P}_{o} \rightarrow \mathcal{G}_{\mathcal{E}, o} \rightarrow \widehat{\mathcal{G}}_{o} \rightarrow 1 .
$$

of completions that is compatible with mixed Hodge structures. Here $\mathcal{G}_{\mathcal{E}, o}$ denotes the completion of $\pi_{1}\left(\mathcal{E}^{\prime}, \vec{v}_{o}\right)$ with respect to the natural homomorphism to $\operatorname{SL}\left(H_{o}\right)$ and $\mathcal{P}_{o}$ the unipotent completion of $\pi_{1}\left(E_{o}^{\prime}, \vec{v}_{o}\right)$. One has exactness on the left as $\mathcal{P}_{o}$ has trivial center. The conjugation action of $\mathcal{G}_{\mathcal{E}, o}$ on $\mathcal{P}_{o}$ induces a homomorphism $\mathfrak{g}_{\mathcal{E}, o} \rightarrow$ Der $\mathfrak{p}_{o}$ of their Lie algebras (cf. 3.10) that is a morphism of MHS.

The tangent vectors $\vec{v}$ induce a section of $\pi_{1}\left(\mathcal{E}^{\prime}, \vec{v}_{o}\right) \rightarrow \pi_{1}\left(\mathcal{M}_{1, \overrightarrow{1}}, x_{o}\right)$. It induces a section of $\widehat{\mathcal{G}}_{o} \rightarrow \mathcal{G}_{\mathcal{E}, o}$ that is compatible with mixed Hodge structures. The natural action of $\widehat{\mathfrak{g}}_{o}$ on $\mathfrak{p}_{o}$ is the composite $\widehat{\mathfrak{g}}_{o} \rightarrow \mathfrak{g} \mathcal{E}, o \rightarrow$ Der $\mathfrak{p}_{o}$ and is therefore a morphism of MHS.

Since $L^{m} \mathfrak{p}_{o}=W_{-m} \mathfrak{p}_{o}$, there is a canonical isomorphism (cf. (2.4))

$$
\mathrm{Gr}_{\bullet}^{W} \mathfrak{p}_{o} \cong \mathbb{L}\left(H_{o}\right) .
$$

of graded Lie algebras in the category of $\mathrm{SL}\left(H_{o}\right)$ modules. The map on each graded quotient is an isomorphism of mixed Hodge structures. 
The element $\sigma$ of $\pi_{1}\left(E_{o}^{\prime}, \vec{v}_{o}\right)$ obtained by rotating the tangent vector once around the identity is trivial in homology and thus lives in the commutator subgroup. The image of its logarithm in

$$
\mathrm{Gr}_{-2}^{W} \mathfrak{p}_{o} \cong \Lambda^{2} H_{o}
$$

is $[\mathbf{a}, \mathbf{b}]$, where $\mathbf{a}, \mathbf{b}$ is any symplectic basis of $H_{1}(E)$. It spans a copy of the trivial representation. Let

$$
\operatorname{Der}^{0} \mathbb{L}\left(H_{o}\right)=\left\{\delta \in \operatorname{Der} \mathbb{L}\left(H_{o}\right): \delta([\mathbf{a}, \mathbf{b}])=0\right\} .
$$

Since the natural action of $\pi_{1}\left(\mathcal{M}_{1, \overrightarrow{1}}, x_{o}\right)$ on $\pi_{1}\left(E_{o}^{\prime}, \vec{v}_{o}\right)$ fixes $\sigma$, we have:

Corollary 15.2. The image of the infinitesimal monodromy representation

$$
\mathrm{Gr}_{\bullet}^{W} \widehat{\mathfrak{u}}_{o} \rightarrow \operatorname{Der} \mathbb{L}\left(H_{o}\right)
$$

lies in $\operatorname{Der}^{0} \mathbb{L}\left(H_{o}\right)$.

The Lie algebra $\mathrm{Gr}_{\bullet}^{W} \widehat{\mathfrak{u}}_{o}$ is freely generated by the image of any $\mathrm{SL}\left(H_{o}\right)$-invariant Hodge section of $\mathrm{Gr}_{\bullet}^{W} \widehat{\mathfrak{u}}_{o} \rightarrow \mathrm{Gr}_{\bullet}^{W} H_{1}\left(\widehat{\mathfrak{u}}_{o}\right)$. Since this projection is an isomorphism in weight -1 , each cuspidal generator $\mathbf{e}_{f}^{\prime}$ and $\mathbf{e}_{f}^{\prime \prime}$ has a canonical lift to $\mathrm{Gr}_{-1}^{W} \widehat{\mathfrak{u}}_{o}$. Fix a lift $\tilde{\mathbf{e}}_{2 n}$ of each Eisenstein generator $\mathbf{e}_{2 n}$ to $\mathrm{Gr}_{\bullet}^{W} \widehat{\mathfrak{u}}_{o}$.

Theorem 15.4. The image of the graded monodromy representation (15.3) is generated as an $\mathrm{SL}\left(H_{o}\right)$-module by the images of the $\tilde{\mathbf{e}}_{2 n}, n \geq 1$.

Proof. First observe that $\operatorname{Gr}_{-1}^{W} \operatorname{Der}^{0} \mathbb{L}\left(H_{o}\right)=0$. This is because

$$
\operatorname{Gr}_{-1}^{W} \mathbb{L}\left(H_{o}\right)=H_{o} \text { and } \operatorname{Gr}_{-2}^{W} \mathbb{L}\left(H_{o}\right)=\mathbb{Q}[\mathbf{a}, \mathbf{b}] .
$$

The element $u \in H_{o}$ corresponds to the derivation $\operatorname{ad}_{u}$. Since $\operatorname{ad}_{u}([\mathbf{a}, \mathbf{b}])=$ $[u,[\mathbf{a}, \mathbf{b}]] \neq 0$ for all non-zero $u \in H_{o}, \operatorname{Gr}_{-1}^{W} \operatorname{Der}^{0} \mathbb{L}(H)=0$.

Since each $\mathbf{e}_{f}^{\prime}$ and $\mathbf{e}_{f}^{\prime \prime}$ in $\mathrm{Gr}_{-1}^{W} \widehat{\mathfrak{u}}_{o}$ has weight -1 , this vanishing implies that $\mathbf{e}_{0}^{j} \cdot \mathbf{e}_{f}^{\prime}$ and $\mathbf{e}_{0}^{j} \cdot \mathbf{e}_{f}^{\prime \prime}$ are in the kernel of the graded monodromy representation. It follows that the image is generated by the images of the remaining generators - the Eisenstein generators $\mathbf{e}_{0}^{j} \cdot \tilde{\mathbf{e}}_{2 n}$.

The next task is to identify the images of the $\tilde{\mathbf{e}}_{2 n}$ in $\operatorname{Der}^{0} \mathbb{L}\left(H_{o}\right)$. For each $n \geq 0$ a basis $\mathbf{v}_{1}, \mathbf{v}_{2}$ of $H$ define derivations $\epsilon_{2 n}\left(\mathbf{v}_{1}, \mathbf{v}_{2}\right)$ by

$$
\epsilon_{2 n}\left(\mathbf{v}_{1}, \mathbf{v}_{2}\right):= \begin{cases}-\mathbf{v}_{2} \frac{\partial}{\partial \mathbf{v}_{1}} & n=0 \\ \operatorname{ad}_{\mathbf{v}_{1}-1}^{2 n-1}\left(\mathbf{v}_{2}\right)-\sum_{\substack{j+k=2 n-1 \\ j>k>0}}(-1)^{j}\left[\operatorname{ad}_{\mathbf{v}_{1}}^{j}\left(\mathbf{v}_{2}\right), \operatorname{ad}_{\mathbf{v}_{1}}^{k}\left(\mathbf{v}_{2}\right)\right] \frac{\partial}{\partial \mathbf{v}_{2}} & n>0 .\end{cases}
$$

Here we are identifying $\mathbb{L}(H)$ with its image in $\operatorname{Der} \mathbb{L}(H)$ under the inclusion ad : $\mathbb{L}(H) \hookrightarrow \operatorname{Der} \mathbb{L}(H)$.

The following result implies that the image of $\tilde{\mathbf{e}}_{2 n}$ in $\operatorname{Der}^{0} \mathbb{L}(H)$ depends only on $\mathbf{e}_{2 n}$ and not on the choice of the lift $\tilde{\mathbf{e}}_{2 n}$.

Proposition 15.6 (Hain-Matsumoto). For each $n \geq 1$ there is a unique copy of $S^{2 n} H(2 n+1)$ in $\mathrm{Gr}_{-2 n-2}^{W} \operatorname{Der}^{0} \mathbb{L}(H)$. It has highest weight vector the derivation $\epsilon_{2 n}\left(\mathbf{v}_{1}, \mathbf{v}_{2}\right)$, where $\mathbf{v}_{1}, \mathbf{v}_{2} \in H$ are non-zero vectors of $\mathfrak{s l}_{2}$-weight 1 and -1 , respectively. 
It follows that the image of $\tilde{\mathbf{e}}_{2 n}$ in $\operatorname{Der} \mathbb{L}\left(H_{o}\right)$ is a multiple (possibly zero) of $\epsilon_{2 n}(\mathbf{b}, \mathbf{a})$. We compute this multiple using the universal elliptic KZB-connection $[6,28,20]$, which provides an explicit formula for the connection on the bundle $\mathfrak{p}$ over $\mathcal{M}_{1, \overrightarrow{1}}$.

Theorem 15.7. For all choices of the lift $\tilde{\mathbf{e}}_{2 n}$, the image of $\tilde{\mathbf{e}}_{2 n}^{B}=2 \pi i \tilde{\mathbf{e}}_{2 n}$ under the graded monodromy representation (15.3) is $2 \epsilon_{2 n}(\mathbf{b}, \mathbf{a}) /(2 n-2)$ ! when $n>0$ and $\epsilon_{0}$ when $n=0$.

Proof. Trivialize the pullback of $\mathcal{H}$ to $\mathbb{C}^{*} \times \mathfrak{h}$ by the sections

$$
T:=\tau \mathbf{a}-\mathbf{b}=\exp \left(\tau \mathbf{e}_{0}\right)(-\mathbf{b}) \text { and } A:=(2 \pi i)^{-1} \mathbf{a} .
$$

In $[20, \S 13-14]$ it is shown that the pullback of $\mathfrak{p}$ to $\mathbb{C}^{*} \times \mathfrak{h}$ may be identified with the trivial bundle

$$
\mathbb{L}(T, A)^{\wedge} \times \mathbb{C}^{*} \times \mathfrak{h} \rightarrow \mathbb{C}^{*} \times \mathfrak{h}
$$

with the connection $\nabla=d+\omega^{\prime}$, where

$$
\omega^{\prime}=-2 \pi i\left(d \tau \otimes \epsilon_{0}(T, A)+\sum_{m \geq 1} \frac{2}{(2 n-2) !} G_{2 n}(\tau) d \tau \otimes \epsilon_{2 n}(T, A)\right) .
$$

To prove the result, we need to rewrite this in terms of the frame $-\mathbf{b}, \mathbf{a}$ of $\mathcal{H}$. First note that $\epsilon_{2 n}\left(c_{1} \mathbf{v}_{1}, c_{2} \mathbf{v}_{2}\right)=c_{1}^{2 n-1} c_{2} \epsilon_{2 n}\left(\mathbf{v}_{1}, \mathbf{v}_{2}\right)$ and that if $g \in \operatorname{SL}(H)$, then $\epsilon_{2 n}\left(g \mathbf{v}_{1}, g \mathbf{v}_{2}\right)=g \cdot \epsilon_{2 n}\left(\mathbf{v}_{1}, \mathbf{v}_{2}\right)$, where $g \in \mathrm{SL}(H)$ acts on a derivation $\delta$ by $g \cdot \delta:=g \delta g^{-1}$. Since $\mathbf{e}_{0} \cdot \mathbf{a}=0$, these imply that

$2 \pi i \epsilon_{2 n}(T, A)=\epsilon_{2 n}(T, \mathbf{a})=\epsilon_{2 n}\left(\exp \left(\tau \mathbf{e}_{0}\right)(-\mathbf{b}), \exp \left(\tau \mathbf{e}_{0}\right) \mathbf{a}\right)=-\exp \left(\tau \mathbf{e}_{0}\right) \cdot \epsilon_{2 n}(\mathbf{b}, \mathbf{a})$.

It follows that $2 \pi i G_{2 n}(\tau) d \tau \otimes \epsilon_{2 n}(T, A)=-\psi_{2 n}\left(\epsilon_{2 n}(\mathbf{b}, \mathbf{a})\right) / 2 \pi i$.

Since the natural connection $\nabla_{0}$ on $\mathcal{H}$ is given by

$$
\nabla_{0}=d-2 \pi i \epsilon_{0}(T, A) \otimes d \tau,
$$

the pullback connection may be written

$$
\begin{aligned}
\nabla & =\nabla_{0}-2 \pi i \sum_{m \geq 1} \frac{2}{(2 n-2) !} G_{2 n}(\tau) d \tau \otimes \epsilon_{2 n}(T, A) \\
& =\nabla_{0}+\sum_{m \geq 1} \frac{2}{(2 n-2) !} \psi_{2 n}\left(\epsilon_{2 m}(\mathbf{b}, \mathbf{a}) / 2 \pi i\right) .
\end{aligned}
$$

It follows that, regardless of the choice of the lifts of the $\tilde{\mathbf{e}}_{2 n}$, the de Rham generator $\tilde{\mathbf{e}}_{2 n}$ goes to $2 \epsilon_{2 n}(\mathbf{b}, \mathbf{a}) / 2 \pi i(2 n-2)$ ! under the graded monodromy representation. Since $\mathbf{e}_{2 n}$ spans a copy of $\mathbb{Q}(1)$, the Betti generator is $\mathbf{e}_{2 n}^{B}=\mathbf{e}_{2 n}^{\mathrm{DR}} / 2 \pi i$.

Remark 15.8. Since $\widehat{\mathfrak{g}}=\mathfrak{g} \oplus \mathbb{Q}(1)$, there are natural representations

$$
\mathfrak{g} \rightarrow \text { Der } \mathfrak{p} \text { and } \operatorname{Gr}_{\bullet}^{W} \mathfrak{g} \rightarrow \operatorname{Der}^{0} \mathbb{L}(H) .
$$

There are also the outer actions

$$
\mathfrak{g} \rightarrow \text { OutDer } \mathfrak{p} \text { and } \mathrm{Gr}_{\bullet}^{W} \mathfrak{g} \rightarrow \operatorname{OutDer} \mathbb{L}(H) .
$$

The representations $\mathrm{Gr}_{\bullet}^{W} \mathfrak{g} \rightarrow \operatorname{Der} \mathbb{L}(H)$ and $\mathrm{Gr}_{\bullet}^{W} \mathfrak{g} \rightarrow$ OutDer $\mathbb{L}(H)$ have the same kernel as $\mathbf{e}_{2} \notin \mathfrak{g}$ and as

$$
\operatorname{InnDer} \mathbb{L}(H) \cap \operatorname{Der}^{0} \mathbb{L}(H)=\mathbb{Q} \epsilon_{2}(\mathbf{b}, \mathbf{a}) .
$$


Exactness of $\mathrm{Gr}_{\bullet}^{W}$ implies that $\mathfrak{g} \rightarrow$ Der $\mathfrak{p}$ and $\mathfrak{g} \rightarrow$ OutDer $\mathfrak{p}$ have the same kernel. Since it is generally easier to work with derivations than with outer derivations, we will work with $\mathfrak{g} \rightarrow$ Der $\mathfrak{p}$.

\section{The Eisenstein Quotient of a Completed Modular Group}

The results of the previous section imply that for all $x \in \mathcal{M}_{1,1}$, each weight graded quotient of the image of $\mathfrak{g}_{x}$ in Der $\mathfrak{p}_{x}$ is a sum of Tate twists $S^{m} H_{x}(r)$ of symmetric powers of $H_{x}$. Any such Lie algebra quotient of $\mathfrak{g}_{x}$ has the property that its weight associated graded is generated by the images of the Eisenstein generators $\mathbf{e}_{2 n}$.

Suppose that $\Gamma$ is a finite index subgroup of $\mathrm{SL}_{2}(\mathbb{Z})$. Denote the completion of $\pi_{1}\left(X_{\Gamma}, x\right)$ with respect to the inclusion $\Gamma \rightarrow \mathrm{SL}_{2}\left(H_{x}\right)$ by $\mathcal{G}_{x}$ and its pronilpotent radical by $\mathcal{U}_{x}$. Denote their Lie algebras by $\mathfrak{g}_{x}$ and $\mathfrak{u}_{x}$, respectively.

Proposition 16.1. For each $x \in X_{\Gamma}$ there is a unique maximal quotient $\mathfrak{g}_{x}^{\text {eis }}$ of $\mathfrak{g}_{x}$ in the category of Lie algebras with a mixed Hodge structure with the property that each weight graded quotient of $\mathfrak{g}_{x}^{\text {eis }}$ is a sum of Tate twists of symmetric powers of $H_{x}$. Moreover, the Lie algebra isomorphism $\mathfrak{g}_{x} \rightarrow \mathfrak{g}_{y}$ corresponding to a path from $x$ to $y$ in $X_{\Gamma}$ induces a Lie algebra (but not a Hodge) isomorphism $\mathfrak{g}_{x}^{\text {eis }} \rightarrow \mathfrak{g}_{y}^{\text {eis }}$. The corresponding local system $\mathfrak{g}^{\mathrm{eis}}:=\left(\mathfrak{g}_{x}^{\mathrm{eis}}\right)_{x \in X_{\Gamma}}$ underlies an admissible VMHS.

The quotient $\mathfrak{g}_{x}^{\text {eis }}$ will be called the Eisenstein quotient of $\mathfrak{g}_{x}$. The corresponding quotient of $\mathcal{G}_{x}$ will be denoted by $\mathcal{G}_{x}^{\text {eis }}$.

Remark 16.2. Note that if we instead use a tangential base point $\vec{v}$, then $H_{\vec{v}}$ is an extension of $\mathbb{Q}$ by $\mathbb{Q}(1)$, so that $\mathfrak{g}_{\vec{v}}^{\text {eis }}$ is a mixed Hodge-Tate structure. (That is, all of its $M_{\bullet}$ weight graded quotients are of type $(p, p)$.) In this case, $\mathfrak{g}_{\vec{v}}^{\text {eis }}$ is the "maximal Tate quotient" of $\mathfrak{g}_{\vec{v}}$ in the category pro-Lie algebras with MHS.

Corollary 16.3. When $\Gamma=\mathrm{SL}_{2}(\mathbb{Z})$, the monodromy homomorphism $\mathfrak{g}_{x} \rightarrow$ Der $\mathfrak{p}_{x}$ factors through $\mathfrak{g}_{x}^{\text {eis }}$ :

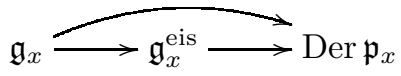

Note that if $\Gamma$ has finite index in $\mathrm{SL}_{2}(\mathbb{Z})$, then the Eisenstein quotient of its relative completion surjects onto the Eisenstein quotient of the relative completion of $\mathrm{SL}_{2}(\mathbb{Z})$.

Proof of Proposition 16.1. If $\mathfrak{h}_{1}$ and $\mathfrak{h}_{2}$ are quotients of $\mathfrak{g}_{x}^{\text {eis }}$ whose weight graded quotients are sums of Tate twists of symmetric powers of $H_{x}$, then the image $\mathfrak{h}$ of $\mathfrak{g}_{x}^{\text {eis }} \rightarrow \mathfrak{h}_{1} \oplus \mathfrak{h}_{2}$ is a quotient of $\mathfrak{g}_{x}^{\text {eis }}$ whose weight graded quotients are sums of twists of symmetric powers of $H_{x}$. It also surjects onto $\mathfrak{h}_{1}$ and $\mathfrak{h}_{2}$. This implies that the "Eisenstein quotients" of $\mathfrak{g}_{x}$ form an inverse system from which it follows that the Eisenstein quotient is unique. Note that since $\mathfrak{s l}\left(H_{x}\right) \cong S^{2} H_{x}, \mathfrak{g}_{x}$ surjects onto $\mathfrak{s l}\left(H_{x}\right)$.

We begin the proof of the second part with an observation. Suppose that $V$ is a MHS and that $K$ is a subspace of $V$ that is defined over $\mathbb{Q}$. Give it the induced weight filtration. Then there is a natural isomorphism

$$
\operatorname{Gr}_{m}^{W}(V / K) \cong\left(\operatorname{Gr}_{m}^{W} V\right) /\left(\operatorname{Gr}_{m}^{W} K\right)
$$


This isomorphism respects the Hodge filtration on each induced from the Hodge filtration $F^{\bullet} \cap\left(W_{m} V\right)$ of $W_{m} V$. These are defined as the images of the maps

$$
\begin{aligned}
F^{p} W_{m} V \rightarrow\left(W_{m} V\right) /\left(W_{m} K\right) \rightarrow & \mathrm{Gr}_{m}^{W}(V / K) \\
& \text { and } F^{p} W_{m} V \rightarrow \mathrm{Gr}_{m}^{W} V \rightarrow\left(\operatorname{Gr}_{m}^{W} V\right) /\left(\operatorname{Gr}_{m}^{W} K\right) .
\end{aligned}
$$

The observation is that if this Hodge filtration defines a Hodge structure on each $\operatorname{Gr}_{m}^{W}(V / K)$, then $K$ is a sub MHS of $V$ and $V / K$ is a quotient MHS of $V$.

Now apply this with $V=\mathfrak{g}_{y}$ and $K$ the ideal of $\mathfrak{g}_{y}$ that corresponds to the kernel of $\mathfrak{g}_{x} \rightarrow \mathfrak{g}_{x}^{\text {eis }}$ under the isomorphism $\mathfrak{g}_{x} \cong \mathfrak{g}_{y}$ given by parallel transport. This implies that $\mathfrak{g}_{y} / K$ is a quotient of $\mathfrak{g}_{y}$ in the category of MHS whose weight graded quotients are sums of twists of symmetric powers of $H_{y}$. It is therefore an Eisenstein quotient of $\mathfrak{g}_{y}$.

The last statement follows from the fact that if $\mathbb{W}$ is a quotient of the local system underlying an admissible variation of MHS $\mathbb{V}$ with the property that each fiber of $W_{x}$ has a MHS that is the quotient of the MHS on $V_{x}$, then $\mathbb{W}$ is an admissible variation of MHS.

The significance of $\mathfrak{g}^{\text {eis }}$ lies in the following result, which follows directly from Theorem 8.3.

Corollary 16.4. For all base points (finite, tangential), the category $\mathrm{HRep}\left(\mathfrak{g}_{x}^{\mathrm{eis}}\right)$ of Hodge representations (Def. 8.2) of $\mathfrak{g}_{x}^{\text {eis }}$ is equivalent to the category of admissible $V M H S$ over $X_{\Gamma}$ whose weight graded quotients are sums of Tate twists $S^{n} \mathbb{H}(r)$ of symmetric powers of $\mathbb{H}$.

Remark 16.5. We will call such variations over a modular curve Eisenstein variations of MHS. Like Tate VMHS in the unipotent case (cf. [26]), Eisenstein variations over a modular curve can be written down reasonably explicitly. This follows from the constructions of Section 8. The explanation we give below is somewhat technical. Suppose that $\mathbb{A}$ is an Eisenstein variation over $X_{\Gamma}$. Set

$$
A_{m, n}=H^{0}\left(X_{\Gamma}, \operatorname{Hom}\left(S^{n} \mathbb{H}, \operatorname{Gr}_{m}^{W} \mathbb{A}\right)\right) .
$$

This is a Tate Hodge structure of weight $m-n$. There is a natural isomorphism

$$
\mathrm{Gr}_{m}^{W} \mathbb{A} \cong \bigoplus_{n} A_{m, n} \otimes S^{n} \mathbb{H}
$$

of MHS. For each $r$ satisfying $0 \leq r \leq \min (n, \ell)$, fix a highest weight vector $\mathbf{h}_{n, \ell}^{(r)}$ of $\mathfrak{s l}_{2}$-weight $n+\ell-2 r$ in $\operatorname{Hom}\left(S^{n} H, S^{\ell} H\right)$, so that

$$
\operatorname{Hom}\left(S^{n} \mathbb{H}, S^{\ell} \mathbb{H}\right) \cong \bigoplus_{r=0}^{\min (n, \ell)} \mathbb{S}^{n+\ell-2 r}\left(\mathbf{h}_{n, \ell}^{(r)}\right) .
$$

Implicit here is that $\mathbf{h}_{n, \ell}^{(r)}$ has Hodge weight $\ell-n$. Set $\mathcal{A}=\mathbb{A} \otimes \mathcal{O}_{X_{\Gamma}}$. Denote the natural connection on it by $\nabla$. Set

$$
\mathcal{A}_{m}=\left(\mathrm{Gr}_{m}^{W} \mathbb{A}\right) \otimes \mathcal{O}_{X_{\Gamma}} \cong \bigoplus_{n} A_{m, n} \otimes S^{n} \mathcal{H}
$$

The standard connection on $\mathcal{H}$ induces a connection on each of these that we denote by $\nabla_{0}$. The construction of Section 8 implies that for each cusp $P \in D$ of $X_{\Gamma}$, there 
are linear maps

$$
\varphi_{k, \ell, P}^{m, n} \in F^{\ell-r+1} W_{2 \ell-2 r+2} \operatorname{Hom}_{\mathbb{C}}\left(A_{m, n}, A_{k, \ell}\right)
$$

such that

$$
(\mathcal{A}, \nabla) \cong\left(\bigoplus_{m} \mathcal{A}_{m}, \nabla_{0}+\Omega\right)
$$

where

$$
\Omega=\sum_{P \in D} \sum_{n, \ell \geq 0} \sum_{r=0}^{\min (n, \ell)} \sum_{m, k} \varphi_{k, \ell, P}^{m, n} \otimes \psi_{n+\ell-2 r+2, P}\left(\mathbf{h}_{n, \ell}^{(r)}\right) .
$$

Implicit in this statement is that the canonical extension $\overline{\mathcal{A}}$ of $(\mathcal{A}, \nabla)$ is isomorphic to

$$
\bigoplus_{m, n} A_{m, n} \otimes\left(S^{n} \overline{\mathcal{H}}, \nabla_{0}\right)
$$

The isomorphism (16.7) is bifiltered with respect to the Hodge and weight filtrations. The condition (16.6) implies that $\Omega \in F^{0} W_{-1} K^{1}\left(\bar{X}_{\Gamma}, D ; \operatorname{End}\left(\operatorname{Gr}_{\bullet}^{W} \mathbb{A}\right)\right)$.

Caution: not every such 1-form $\Omega$ defines the structure of an admissible variation of MHS over $X_{\Gamma}$. The issue is that one needs the monodromy of $\left(\mathcal{A}, \nabla_{0}+\Omega\right)$ to be defined over $\mathbb{Q}$. Determining which $\Omega$ give rise to Eisenstein variations is closely related to the problem of determining the relations in $\mathfrak{u}^{\text {eis }}$.

\section{Modular Symbols and Pollack's Quadratic Relations}

Motivic arguments (cf. [23]) suggested that $\mathfrak{u}_{x}^{\text {eis }}$ may not be freely generated by the $\mathbf{e}_{0}^{j} \cdot \mathbf{e}_{2 m}$ and predict that the relations that hold between the $\mathbf{e}_{0}^{j} \cdot \mathbf{e}_{m}$ arise from cusp forms. In other words, cusp forms go from being generators of $\mathfrak{u}_{x}$ to relations in $\mathfrak{u}_{x}^{\text {eis }}$. The goal of the rest of this paper is to sketch a Hodge theoretic explanation for these relations. For this we will need to recall some basic facts about modular symbols, which record the periods of cusp forms and determine the Hodge structure on $H_{\text {cusp }}^{1}\left(\mathcal{M}_{1,1}, S^{2 n} \mathbb{H}\right)$. In this and subsequent sections, $\mathfrak{B}_{w}=\mathfrak{B}_{w}\left(\mathrm{SL}_{2}(\mathbb{Z})\right)$, the set of normalized Hecke eigen cusp forms of $\mathrm{SL}_{2}(\mathbb{Z})$.

17.1. Modular Symbols. Modular symbols are homogeneous polynomials attached to cusp forms of $\mathrm{SL}_{2}(\mathbb{Z})$. They play two roles: they give a concrete representation of the cohomology class associated to a cusp form; secondly, modular symbols of degree $m$ record the periods of the MHS on $H^{1}\left(\mathcal{M}_{1,1}, S^{m} \mathbb{H}\right) \cong$ $H^{1}\left(\mathrm{SL}_{2}(\mathbb{Z}), S^{m} H\right)$. A standard reference is [27, Ch. IV].

Recall that $\mathrm{SL}_{2}(\mathbb{Z})$ has presentation

$$
\mathrm{SL}_{2}(\mathbb{Z})=\left\langle S, U: S^{2}=U^{3}, S^{4}=U^{6}=I\right\rangle .
$$

where

$$
S=\left(\begin{array}{cc}
0 & -1 \\
1 & 0
\end{array}\right), \quad T=\left(\begin{array}{ll}
1 & 1 \\
0 & 1
\end{array}\right), \quad U:=S T=\left(\begin{array}{cc}
0 & -1 \\
1 & 1
\end{array}\right)
$$

The action of $\mathrm{SL}_{2}(\mathbb{Z})$ on $\mathfrak{h}$ factors through

$$
\mathrm{PSL}_{2}(\mathbb{Z}):=\mathrm{SL}_{2}(\mathbb{Z}) /( \pm I)=\left\langle S, U: S^{2}=U^{3}=I\right\rangle .
$$


17.1.1. Group cohomology. Suppose that $\Gamma$ is a group and that $V$ is a left $\Gamma$-module. Then one has the complex

$$
0 \longrightarrow C^{0}(\Gamma, V) \stackrel{\delta}{\longrightarrow} C^{1}(\Gamma, V) \stackrel{\delta}{\longrightarrow} C^{2}(\Gamma, V) \stackrel{\delta}{\longrightarrow} \cdots
$$

of standard cochains, where $C^{j}(\Gamma, V)=\left\{\right.$ functions $\left.\phi: \Gamma^{j} \rightarrow V\right\}$. The differential takes $v \in V=C^{0}(\Gamma, V)$ to the function $\delta v: \gamma \mapsto(\gamma-1) v$ and $\delta: C^{0}(\Gamma, V) \rightarrow$ $C^{1}(\Gamma, V)$ takes $\phi: \Gamma \rightarrow V$ to the function

$$
(\delta \phi)\left(\gamma_{1}, \gamma_{2}\right) \mapsto \phi\left(\gamma_{1}\right)-\phi\left(\gamma_{1} \gamma_{2}\right)+\gamma_{1} \cdot \phi\left(\gamma_{2}\right) .
$$

So $\phi$ is a 1-cocycle if and only if $\phi\left(\gamma_{1} \gamma_{2}\right)=\phi\left(\gamma_{1}\right)+\gamma_{1} \cdot \phi\left(\gamma_{2}\right)$. The cohomology $H^{\bullet}(\Gamma, V)$ of $\Gamma$ with coefficients in $V$ is defined to be the homology of this complex.

Now suppose that $V$ is a real or complex vector space and that $\Gamma$ acts on a simply connected manifold $X$. Fix a base point $x_{o} \in X$. As in Proposition 4.3, to each $\gamma \in G$ we can associate the unique homotopy class $c_{\gamma}$ of paths in $X$ from $x_{o}$ to $\gamma \cdot x_{o}$. If $\omega \in E^{1}(X) \otimes V$ is $\Gamma$ invariant, then the function

$$
\phi: \gamma \mapsto \int_{c_{\gamma}} \omega
$$

is a 1-cocycle. Changing the base point from $x_{o}$ to $x^{\prime}$ changes $\phi$ by the coboundary of $\int_{x_{o}}^{x^{\prime}} \omega \in V$. (Cf. Remark 4.4.) This construction induces a map

$$
H^{1}\left(E^{1}(X \times V)^{\Gamma}\right) \rightarrow H^{1}(\Gamma, V),
$$

which is an isomorphism when $\Gamma$ acts properly discontinuously and virtually freely on $X$. This is the case when $\Gamma$ is a modular group and $X$ is the upper half plane.

Suppose that $V$ is divisible as an abelian group. When $-I$ acts trivially on $V$, $V$ is the pullback of a $\mathrm{PSL}_{2}(\mathbb{Z})$-module and

$$
H^{1}\left(\mathrm{PSL}_{2}(\mathbb{Z}), V\right) \rightarrow H^{1}\left(\mathrm{SL}_{2}(\mathbb{Z}), V\right)
$$

is an isomorphism.

17.1.2. Cuspidal cohomology. Suppose that $F$ is a field of characteristic zero. Set $H_{F}=F \mathbf{a} \oplus F \mathbf{b}$. Define $C_{\text {cusp }}^{\bullet}\left(\mathrm{SL}_{2}(\mathbb{Z}), S^{2 n} H_{F}\right)$ to be the kernel of the restriction mapping

$$
C^{\bullet}\left(\mathrm{SL}_{2}(\mathbb{Z}), S^{2 n} H_{F}\right) \rightarrow \widetilde{C}^{\bullet}\left(\langle T\rangle, S^{2 n} H_{F}\right)
$$

where the right hand complex is the quotient of $C^{\bullet}\left(\langle T\rangle, S^{2 n} H_{F}\right)$ by $\mathbf{a}^{2 n}$ in degree 0 . Set

$$
H_{\text {cusp }}^{\bullet}\left(\mathrm{SL}_{2}(\mathbb{Z}), S^{2 n} H_{F}\right):=H^{\bullet}\left(C_{\text {cusp }}^{\bullet}\left(\mathrm{SL}_{2}(\mathbb{Z}), S^{2 n} H_{F}\right)\right) .
$$

The corresponding long exact sequence gives the exact sequence

$$
\begin{aligned}
0 \rightarrow H_{\text {cusp }}^{1}\left(\mathrm{SL}_{2}(\mathbb{Z}), S^{2 n} H_{F}\right) & \rightarrow H^{1}\left(\mathrm{SL}_{2}(\mathbb{Z}), S^{2 n} H_{F}\right) \\
& \rightarrow H^{1}\left(\langle T\rangle, S^{2 n} H_{F}\right) \rightarrow H_{\text {cusp }}^{2}\left(\mathrm{SL}_{2}(\mathbb{Z}), S^{2 n} H_{F}\right) \rightarrow 0 .
\end{aligned}
$$

This is an instance of the exact sequences (6.3) and (11.3) where $C^{\prime}=\mathcal{M}_{1,1}$.

The cuspidal cohomology group $H_{\text {cusp }}^{1}\left(\mathrm{SL}_{2}(\mathbb{Z}), S^{2 n} H_{F}\right)$ has a nice description. Recall that $\mathrm{SL}_{2}(\mathbb{Z})$ acts on $H$ via the formula (10.1) with $\mathbf{v}_{1}=-\mathbf{b}$ and $\mathbf{v}_{2}=\mathbf{a}$. 
Proposition 17.1. Suppose that $F$ is a field of characteristic zero. For all $n \geq 1$, there is an isomorphism

$$
H_{\text {cusp }}^{1}\left(\mathrm{SL}_{2}(\mathbb{Z}), S^{2 n} H_{F}\right)
$$

with the vector space of $r(\mathbf{a}, \mathbf{b}) \in S^{2 n} H_{F}$ that satisfy

$$
(I+S) r(\mathbf{a}, \mathbf{b})=0 \text { and }\left(I+U+U^{2}\right) r(\mathbf{a}, \mathbf{b})=0
$$

modulo $\mathbf{a}^{2 n}-\mathbf{b}^{2 n}$.

Proof. Suppose that $\phi: \mathrm{SL}_{2}(\mathbb{Z}) \rightarrow S^{2 n} H_{F}$ is a cuspidal 1-cocycle. Since $-I$ acts trivially on $S^{2 n} H$, the cocycle condition and the equation $(-I)^{2}=1$ imply that $\phi(-I)=0$. Since $S$ and $U$ generate $\mathrm{SL}_{2}(\mathbb{Z}), \phi$ is determined by $\phi(S)$. Since $U=S T$,

$$
\phi(U)=\phi(S T)=\phi(S)+S \phi(T)=\phi(S) .
$$

Thus $\phi$ is determined by $\phi(S)$. Denote this element of $S^{2 n} H_{F}$ by $\mathbf{r}_{\phi}(\mathbf{a}, \mathbf{b})$. Since $S^{2}=U^{3}=I$, the cocycle condition implies that $r_{f}(\mathbf{a}, \mathbf{b})$ satisfies the equation (17.2). Conversely, if $r(\mathbf{a}, \mathbf{b}) \in S^{2 n} H_{F}$ satisfies these equations, it determines a well-defined cuspidal cocycle $\phi$ by $\phi(S)=\phi(U)=r(\mathbf{a}, \mathbf{b})$.

The last statement follows as the only cuspidal coboundaries are scalar multiplies of $\delta \mathbf{a}^{2 n}$. This has value $\mathbf{r}(\mathbf{a}, \mathbf{b})=\mathbf{b}^{2 n}-\mathbf{a}^{2 n}$ on $S$.

Remark 17.3. Since $(I+S) \sum_{j} c_{j} \mathbf{a}^{j} \mathbf{b}^{2 n-j}$ vanishes if and only if $c_{2 n-j}=(-1)^{j+1} c_{j}$ for all $j$, the terms of a cocycle $r(\mathbf{a}, \mathbf{b})$ of top degree in $\mathbf{a}$ and $\mathbf{b}$ is a multiple of $\mathbf{a}^{2 n}-\mathbf{b}^{2 n}$. Since this corresponds to the coboundary of $\mathbf{a}^{2 n}$, we can identify $H_{\text {cusp }}^{1}\left(\mathrm{SL}_{2}(\mathbb{Z}), S^{2 n} H_{F}\right)$ with those $\mathbf{r}(\mathbf{a}, \mathbf{b})$ that satisfy the cocycle conditions (17.2) and have no terms of degree $2 n$ in $\mathbf{a}$ or $\mathbf{b}$.

17.1.3. Modular symbols. If $f$ is a cusp form of weight $2 n+2$, the $S^{2 n} H$-valued 1 -form $\omega_{f}\left(\mathbf{b}^{2 n}\right)$ is $\mathrm{SL}_{2}(\mathbb{Z})$-invariant. Since $f$ is a cusp form, it is holomorphic on the $q$-disk. We can therefore take the base point $x_{o}$ above to be the cusp $q=0$. Since $T$ fixes $q=0$, the function

$$
\gamma \mapsto \int_{x_{o}}^{\gamma x_{o}} \omega_{f}\left(\mathbf{w}^{2 n}\right)=(2 \pi i)^{2 n} \int_{x_{o}}^{\gamma x_{o}} \omega_{f}\left(\mathbf{b}^{2 n}\right) \in S^{2 n} H_{\mathbb{C}}
$$

is a well defined cuspidal 1-cocycle. The modular symbol of $f$ is its value ${ }^{23}$

$$
\mathrm{r}_{f}(\mathbf{a}, \mathbf{b}):=\int_{0}^{1} f(q) \mathbf{w}^{2 n} \frac{d q}{q}=-(2 \pi i)^{2 n+1} \int_{0}^{\infty} f(i y)(\mathbf{b}-i y \mathbf{a})^{2 n} d(i y) \in S^{2 n} H_{\mathbb{C}}
$$

on $S$. It satisfies the cocycle condition (17.2) and represents the class

$$
(2 \pi i)^{2 n} \omega_{f}\left(\mathbf{b}^{2 n}\right) \in H_{\text {cusp }}^{1}\left(\mathcal{M}_{1,1}, S^{2 n} \mathbb{H}\right) .
$$

It determines $f$.

\footnotetext{
${ }^{23}$ We use this normalization because, if $f \in \mathfrak{B}_{2 n+2}$, then $f(q) \mathbf{w}^{2 n} d q / q \in H_{\mathrm{DR}}^{1}\left(\mathcal{M}_{1,1 / \mathbb{Q}}, S^{2 n} \mathcal{H}\right)$. Cf. $[20, \S 21]$.
} 
17.2. Hodge theory. The Hodge structure

$$
H_{\text {cusp }}^{1}\left(\mathcal{M}_{1,1}, S^{2 n} \mathbb{H}\right)=H^{2 n+1,0} \oplus H^{0,2 n+1} .
$$

has a description in terms of modular symbols. The underlying $\mathbb{Q}$ vector space is the set of $r(\mathbf{a}, \mathbf{b}) \in S^{2 n} H_{\mathbb{Q}}$ that satisfy (17.2), modulo $\mathbf{a}^{2 n}-\mathbf{b}^{2 n}$; the Hodge filtration is given by

$$
H^{2 n+1,0}=F^{2 n+1} H_{\text {cusp }}^{1}\left(\mathcal{M}_{1,1}, S^{2 n} \mathbb{H}\right)=\left\{\mathbf{r}_{f}(\mathbf{a}, \mathbf{b}): f \in M_{2 n+2}^{o}\right\} / \mathbb{C}\left(\mathbf{a}^{2 n}-\mathbf{b}^{2 n}\right) .
$$

There is more structure. Each element $r(\mathbf{a}, \mathbf{b})$ of $S^{2 n} H$ can be written in the form

$$
r(\mathbf{a}, \mathbf{b})=r^{+}(\mathbf{a}, \mathbf{b})+r^{-}(\mathbf{a}, \mathbf{b}),
$$

where $\mathbf{r}^{+}(\mathbf{a}, \mathbf{b})$ is the sum of the terms involving only even powers of $\mathbf{a}$ and $\mathbf{b}$ and $\mathbf{r}^{-}(\mathbf{a}, \mathbf{b})$ is the sum of the terms involving only odd powers.

If $f$ has real Fourier coefficients (e.g., $f \in \mathfrak{B}_{2 n+2}$ ), then

$$
\mathrm{r}_{f}(\mathbf{a}, \mathbf{b})=\mathrm{r}_{f}^{+}(\mathbf{a}, \mathbf{b})+i \mathrm{r}_{f}^{-}(\mathbf{a}, \mathbf{b})
$$

where $\mathbf{r}_{f}^{ \pm}(\mathbf{a}, \mathbf{b})$ are real. Since the cocycle corresponding to $\bar{f}$ is $\mathbf{r}_{f}^{+}(\mathbf{a}, \mathbf{b})-i \mathbf{r}_{f}^{-}(\mathbf{a}, \mathbf{b})$, $\mathbf{r}_{f}^{+}(\mathbf{a}, \mathbf{b}), \mathbf{r}_{f}^{-}(\mathbf{a}, \mathbf{b}) \in S^{2 n} H_{\mathbb{R}}$ also satisfy the cocycle condition. Since the classes $\omega_{f}\left(\mathbf{b}^{2 n}\right), f \in \mathfrak{B}_{2 n+2}$ span the cuspidal cohomology, we deduce:

Proposition 17.5. If $r(\mathbf{a}, \mathbf{b}) \in S^{2 n} H_{F}$ satisfies the cocycle condition (17.2), then so do $\mathrm{r}^{+}(\mathbf{a}, \mathbf{b})$ and $\mathrm{r}^{-}(\mathbf{a}, \mathbf{b})$.

This gives a decomposition $V_{F}=V_{F}^{+} \oplus V_{F}^{-}$of $V_{F}:=H_{\text {cusp }}^{1}\left(\mathcal{M}_{1,1}, S^{2 n} \mathbb{H}_{F}\right)$. Since

$$
H_{\text {cusp }}^{2 n+1,0}\left(\mathcal{M}_{1,1}, S^{2 n} \mathbb{H}\right) \cap H^{1}\left(\mathcal{M}_{1,1}, S^{2 n} \mathbb{H}_{\mathbb{R}}\right)=0
$$

both parts $r_{f}^{ \pm}(\mathbf{a}, \mathbf{b})$ of the modular symbol of a cusp form with real Fourier coefficients are non-zero. In particular, for each $f \in \mathfrak{B}_{2 n+2}$ we can write

$$
V_{f, \mathbb{R}}=V_{f, \mathbb{R}}^{+} \oplus V_{f, \mathbb{R}}^{-}:=\mathbb{R} \mathrm{r}_{f}^{+}(\mathbf{a}, \mathbf{b}) \oplus \mathbb{R} \mathrm{r}_{f}^{-}(\mathbf{a}, \mathbf{b}) .
$$

Note that $V_{f}^{+}$and $V_{f}^{-}$are real sub Hodge structures of $V_{f}$.

17.2.1. The action of real Frobenius. Complex conjugation (aka "real Frobenius") $\mathcal{F}_{\infty} \in \operatorname{Gal}(\mathbb{C} / \mathbb{R})$ acts on $\mathcal{M}_{1,1}$ and on the local system $\mathbb{H}_{\mathbb{R}}$ as we shall explain below. It therefore acts on $V_{\mathbb{R}}=H_{\text {cusp }}^{1}\left(\mathcal{M}_{1,1}, S^{2 n} \mathbb{H}_{\mathbb{R}}\right)$. In this section we show that its eigenspaces are $V_{\mathbb{R}}^{ \pm}$.

The stack $\mathcal{M}_{1,1 / \mathbb{C}}$ has a natural real (even $\mathbb{Q}$ ) form, viz, $\mathbb{G}_{m} \backslash\left(\mathbb{A}_{\mathbb{R}}^{2}-D\right)$, where $D$ is the discriminant locus $u^{3}-27 v^{2}=0$ and where $t \cdot(u, v)=\left(t^{4} u, t^{6} v\right)$. The involution $\mathcal{F}_{\infty}:(u, v) \mapsto(\bar{u}, \bar{v})$ of $\mathcal{M}_{1,1}$ is covered by the involution of $\tau \mapsto-\bar{\tau}$ of $\mathfrak{h}$.

The universal curve $\mathcal{E}$ over it also has a natural real form as it is defined over $\mathbb{R}$. The projection $\mathcal{E} \rightarrow \mathcal{M}_{1,1}$ is invariant under complex conjugation. This implies that $\mathcal{F}_{\infty}$ acts on $\mathbb{H}_{\mathbb{R}}$. This action is determined by the action of $\mathcal{F}_{\infty}$ on $H$, the fiber over the tangent vector $\partial / \partial q$, which is real and therefore fixed by $\mathcal{F}_{\infty}$. This induced map is easily seen to be the involution $\sigma: H \rightarrow H$ defined by ${ }^{24}$

$$
\mathbf{b} \mapsto-\mathbf{b}, \mathbf{a} \mapsto \mathbf{a} .
$$

\footnotetext{
${ }^{24}$ Here $H$ is identified with $H^{1}\left(E_{\partial / \partial q}\right)$ which is isomorphic to $H_{1}\left(E_{\partial / \partial q}\right)(-1)$. So the actions of $\mathcal{F}_{\infty}$ on $H_{1}\left(E_{\partial / \partial q}\right)$ and $H^{1}\left(E_{\partial / \partial q}\right)$ differ by -1 .
} 
The monodromy representation $\mathrm{SL}_{2}(\mathbb{Z}) \rightarrow$ Aut $H$ of $\mathcal{F}_{\infty}^{*} \mathbb{H}$ is the standard representation conjugated by $\sigma$. There is therefore a natural action

$$
\mathcal{F}_{\infty}: H_{\text {cusp }}^{1}\left(\mathcal{M}_{1,1}, S^{2 n} \mathbb{H}_{\mathbb{R}}\right) \rightarrow H_{\text {cusp }}^{1}\left(\mathcal{M}_{1,1}, S^{2 n} \mathbb{H}_{\mathbb{R}}\right) .
$$

Let $\overline{\mathcal{F}}_{\infty}: H_{\text {cusp }}^{1}\left(\mathcal{M}_{1,1}, S^{2 n} \mathbb{H}_{\mathbb{C}}\right) \rightarrow H_{\text {cusp }}^{1}\left(\mathcal{M}_{1,1}, S^{2 n} \mathbb{H}_{\mathbb{C}}\right)$ be its composition with complex conjugation $H_{\text {cusp }}^{1}\left(\mathcal{M}_{1,1}, S^{2 n} \mathbb{H}_{\mathbb{C}}\right) \rightarrow H_{\text {cusp }}^{1}\left(\mathcal{M}_{1,1}, S^{2 n} \mathbb{H}_{\mathbb{C}}\right)$. This is the de Rham involution.

Lemma 17.7. Real Frobenius $\mathcal{F}_{\infty}$ acts on $V_{\mathbb{R}}=H_{\text {cusp }}^{1}\left(\mathcal{M}_{1,1}, S^{2 n} \mathbb{H}_{\mathbb{R}}\right)$ by multiplication by +1 on $V_{\mathbb{R}}^{+}$and -1 on $V_{\mathbb{R}}^{-}$.

Proof. The result follows from equations (17.4) and (17.6) and the fact that the image of $F^{2 n+1} H_{\mathrm{DR}}^{1}\left(\mathcal{M}_{1,1 / \mathbb{R}}, S^{2 n} \mathcal{H}\right)$ in $H_{\text {cusp }}^{1}\left(\mathcal{M}_{1,1}, S^{2 n} \mathbb{H}_{\mathbb{C}}\right)$ is invariant under $\overline{\mathcal{F}}_{\infty}$.

17.2.2. Extensions of $M H S$ associated to cusp forms. Since $V_{f, \mathbb{C}}=V_{f, \mathbb{R}} \oplus F^{r} V_{f}$ when $r<2 n+2$ and $F^{0} V_{f}(r)=0$ when $r \geq 2 n+2$, we have

$$
\operatorname{Ext}_{\mathrm{MHS}}^{1}\left(\mathbb{R}, V_{f}(r)\right) \cong \begin{cases}0 & r<2 n+2, \\ i V_{f, \mathbb{R}} & r \geq 2 n+2 \text { even, } \\ V_{f, \mathbb{R}} & r \geq 2 n+2 \text { odd. }\end{cases}
$$

We can now compute the extensions invariant under the de Rham involution.

Proposition 17.9. If $f \in \mathfrak{B}_{2 n+2}$, then

$$
\operatorname{Ext}_{\mathrm{MHS}}^{1}\left(\mathbb{R}, V_{f}(r)\right)^{\overline{\mathcal{F}}_{\infty} \cong} \cong \begin{array}{ll}
0 & r<2 n+2, \\
i V_{f, \mathbb{R}}^{-} & r \geq 2 n+2 \text { even }, \\
V_{f, \mathbb{R}}^{+} & r \geq 2 n+2 \text { odd } .
\end{array}
$$

In particular, it is one dimensional for all $r \geq 2 g+2$.

Proof. Since $\mathcal{F}_{\infty}: V_{f, \mathbb{C}} \rightarrow V_{f, \mathbb{C}}$ is $\mathbb{C}$-linear and since twisting by $\mathbb{R}(r)$ multiplies this action by $(-1)^{r}$, Lemma 17.7 implies that $\overline{\mathcal{F}}_{\infty}$ acts on $i V_{\mathbb{R}}^{+}(r)$ by multiplication by $(-1)^{r+1}$ and on $i V_{\mathbb{R}}^{-}$by $(-1)^{r}$. The result follows from the $\overline{\mathcal{F}}_{\infty}$-equivariant isomorphism $\operatorname{Ext}_{\mathrm{MHS}}^{1}\left(\mathbb{R}, V_{f}(r)\right) \cong i V_{f, \mathbb{R}}(r)$ which holds for all $r \geq 2 n+2$.

So each normalized Hecke eigen cusp form $f \in \mathfrak{B}_{2 n+2}$ determines an element of $\operatorname{Ext}_{\mathrm{MHS}}^{1}\left(\mathbb{R}, V_{f}(r)\right)^{\overline{\mathcal{F}}_{\infty}}$ for each $r \geq 2 n+2$. Namely, the extension corresponding to $i \mathrm{r}_{f}^{+}(\mathbf{a}, \mathbf{b}) \in i V_{f, \mathbb{R}}^{+}$when $r$ is even, and $\mathrm{r}_{f}^{-}(\mathbf{a}, \mathbf{b}) \in V_{f, \mathbb{R}}^{-}$when $r$ is odd.

17.3. Pollack's quadratic relations. Motivic considerations [23] suggest that $\mathfrak{u}_{x}^{\text {eis }}$ is not free and that a minimal set of relations that hold between the $\mathbf{e}_{0}^{j} \cdot \mathbf{e}_{2 n}$ are parametrized by cusp forms. In fact, each cusp form should determine relations between the $\mathbf{e}_{0}^{j} \cdot \mathbf{e}_{2 n}$ of every degree $\geq 2$. (For this purpose, $\mathbf{e}_{0}$ is considered to have degree 1.) One way to guess such relations is to find relations that hold

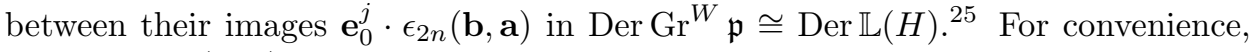
set $\epsilon_{2 n}=\epsilon_{2 n}(\mathbf{b}, \mathbf{a})$.

In his undergraduate thesis [33], Pollack found a complete set of quadratic relations that hold between the $\epsilon_{2 n}$, and found relations of all degrees $>2$ that hold between the $\mathbf{e}_{0}^{j} \cdot \epsilon_{2 n}$ modulo a certain filtration of $\operatorname{Der} \mathbb{L}(H)$. Here we state his quadratic relations.

\footnotetext{
${ }^{25} \mathrm{Since} \mathrm{Gr}^{W}$ is exact, one neither gains nor loses relations in the associated graded.
} 
Theorem 17.10 (Pollack [33, Thm. 2]). The relation

$$
\sum_{\substack{j+k=n \\ j, k>0}} c_{j}\left[\epsilon_{2 j+2}, \epsilon_{2 k+2}\right]=0
$$

holds in $\operatorname{Der} \mathbb{L}(H)$ if and only if there is a cusp form $f$ of weight $2 n+2$ whose modular symbol is

$$
r_{f}^{+}(\mathbf{a}, \mathbf{b})=\sum_{\substack{j+k=n \\ j, k \geq 0}} c_{j} \mathbf{a}^{2 j} \mathbf{b}^{2 n-2 j} .
$$

In view of Theorem 15.7, this suggests each cusp form of weight $2 n+2$ with $r_{f}^{+}(\mathbf{a}, \mathbf{b})=\sum c_{j} \mathbf{a}^{2 j} \mathbf{b}^{2 n-2 j}$ might determine a relation

$$
\sum_{\substack{j+k=n \\ j, k>0}} c_{j}(2 j) !(2 k) !\left[\mathbf{e}_{2 j+2}, \mathbf{e}_{2 k+2}\right]=0
$$

in $\operatorname{Gr}_{\bullet}^{W} \mathfrak{u}_{x}^{\text {eis }}$ and that these relations are connected with $\operatorname{Ext}_{\mathrm{MHS}}^{1}\left(\mathbb{R}, V_{f}(2 r)\right)$ for appropriate $r>0$. In the remaining sections, we show that Pollack's relations do indeed lift to relations in $\mathrm{Gr}_{\bullet}^{W} \mathfrak{u}_{x}^{\text {eis }}$ and explain the connection to extensions of MHS related to cusp forms. In preparation for proving this, we restate Pollack's result in cohomological terms.

We let the base point be $\vec{v}=\partial / \partial q$, although any base point will do. Denote the image of the monodromy homomorphism $\mathcal{G} \rightarrow$ Aut $\mathfrak{p}$ by $\mathcal{D}$ and the Lie algebra of its pronilpotent radical by $\mathfrak{n}$. Since the monodromy homomorphism is a morphism of MHS, $\mathcal{O}(\mathcal{D})$ and $\mathfrak{n}$ have natural MHSs. This implies that $H^{\bullet}\left(\mathcal{D}, S^{m} H(r)\right)$ has a natural MHS for each $m \geq 0$ and $r \in \mathbb{Z}$ and that the natural isomorphism

$$
H^{\bullet}\left(\mathcal{D}, S^{m} H(r)\right) \cong\left[H^{\bullet}(\mathfrak{n}) \otimes S^{m} H(r)\right]^{\mathrm{SL}(H)}
$$

is an isomorphism of MHS. (Cf. [21].)

Since the monodromy representation factors through $\mathcal{G}^{\text {eis }}$, there is an MHS isomorphism

$$
H_{1}(\mathfrak{n}) \cong \bigoplus_{n>0} S^{2 n}\left(\epsilon_{2 n+2}\right) \cong \bigoplus_{n>0} S^{2 n} H(2 n+1) .
$$

This implies that

$$
H^{1}\left(\mathcal{D}, S^{2 n} H(2 n+1)\right)=\operatorname{Hom}_{\mathrm{SL}(H)}\left(H_{1}(\mathfrak{n}), S^{n} H\right)(2 n+1) \cong \mathbb{Q}(0) .
$$

Regard $S^{2 m} H=S^{2 m}\left(\mathbf{b}^{2 m}\right)$. Let $\check{\epsilon}_{2 m}$ be the element of

$$
H^{1}\left(\mathcal{D}, S^{2 m} H\right) \cong \operatorname{Hom}_{\mathrm{SL}(H)}\left(H_{1}(\mathfrak{u}), S^{2 m} H\right)
$$

that takes the class of $\epsilon_{2 m+2}$ to $\mathbf{b}^{2 m}$.

The standard duality (Prop. 2.2) between quadratic relations in $\mathfrak{n}$ and the cup product $H^{1}(\mathfrak{n}) \otimes H^{1}(\mathfrak{n}) \rightarrow H^{2}(\mathfrak{n})$ gives the following dual version of Pollack's quadratic relations, Theorem 17.10.

Proposition 17.12. There is a surjection

$$
H^{2}\left(\mathcal{D}, S^{2 n} H\right) \rightarrow H_{\text {cusp }}^{1}\left(\mathcal{M}_{1,1}, S^{2 n} \mathbb{H}_{\mathbb{Q}}\right)^{\overline{\mathcal{F}}_{\infty}} \otimes \mathbb{Q}(-2 n-2)
$$


which is a morphism of MHS when $H_{\text {cusp }}^{1}\left(\mathcal{M}_{1,1}, S^{2 n} \mathbb{H}\right)^{\overline{\mathcal{F}}_{\infty}}$ is regarded as a HS of type $(0,0)$. After tensoring with $\mathbb{R}$, it gives a surjection

$$
H^{2}\left(\mathcal{D}, S^{2 n} H_{\mathbb{R}}\right) \rightarrow \bigoplus_{f \in \mathfrak{B}_{2 n+2}} \mathbb{R}(-2 n-2)
$$

The generator of $\mathbb{R}(-2 n-2)$ corresponding to $f \in \mathfrak{B}_{2 n+2}$ will be denoted by $z_{f}$. When $j+k=n$, the composition of the cup product

$$
H^{1}\left(\mathcal{D}, S^{2 j} H(2 j+1)\right) \otimes H^{1}\left(\mathcal{D}, S^{2 k} H(2 k+1)\right) \rightarrow H^{2}\left(\mathcal{D}, S^{2 n} H(2 n+2)\right)
$$

with the projection (17.13) is given by

$$
\check{\epsilon}_{2 j} \otimes \check{\epsilon}_{2 k} \rightarrow \sum_{f \in \mathfrak{B}_{2 n+2}} c_{j, k}(f) z_{f}
$$

where $\mathbf{r}_{f}^{+}(\mathbf{a}, \mathbf{b})=\sum_{j+k=n} c_{j, k}(f) \mathbf{a}^{2 j} \mathbf{b}^{2 k}$.

The reason that $V_{f}^{-}$appears in $H^{2}$ and the coefficients of elements of $V_{f}^{+}$occur in the relations is explained by noting that the Petersson inner product induces an isomorphism $V_{f}^{-}\left(V_{f}^{+}\right)^{*}$, while relations give subspaces of $H_{2}$.

Remark 17.15. The occurrence of cusp forms here (without their associated Hodge structure) should be related to, and may help explain, the appearance of modular and cusp forms in the work of Conant, Kassabov and Vogtmann $[9,10,8]$ on the $\mathrm{Sp}(H)$-representation theory of the derivation algebra of a once punctured Riemann surface $S$ of genus $g \gg 0$.

\section{Deligne Cohomology and Extensions of VMHS}

The relations that hold in $\mathfrak{u}_{x}^{\text {eis }}$ are controlled by relations in the Yoneda ext groups of the category $\operatorname{MHS}\left(\mathcal{M}_{1,1}, \mathbb{H}\right)$. In this section, we sketch the relationship between Deligne cohomology of the relative completion of the fundamental group of an affine curve $C^{\prime}=C-D$ and Yoneda ext groups in the categories $\operatorname{MHS}\left(C^{\prime}, \mathbb{H}\right)$. This generalizes the results of [5] that hold in the unipotent case. We will work in the category of $\mathbb{Q}$-MHS, although the discussion is equally valid in the category of $\mathbb{R}$-MHS. This section is a summary of results from [21] where full details can be found.

18.1. Deligne-Beilinson cohomology of a curve. Let $\mathbb{V}$ be a PVHS over the affine (orbi) curve $C^{\prime}=C-D$. The Deligne-Beilinson cohomology $H_{\mathcal{D}}^{\bullet}\left(C^{\prime}, \mathbb{V}\right)$ is the cohomology of the complex

$$
\begin{aligned}
\operatorname{cone}\left(F^{0} W_{0} \operatorname{Dec}_{W} K_{\mathbb{C}}^{\bullet}(C, D ; \mathbb{V}) \oplus W_{0} \operatorname{Dec}_{W} K_{\mathbb{Q}}^{\bullet}(C, D ; \mathbb{V})\right. \\
\left.\quad \rightarrow W_{0} \operatorname{Dec}_{W} K_{\mathbb{C}}^{\bullet}(C, D ; \mathbb{V})\right)[-1] .
\end{aligned}
$$

Here $\operatorname{Dec}_{W}$ is Deligne's filtration decalée functor (with respect to $W_{\bullet}$ ), which is defined in $[11, \S 1.3]$. The DB-cohomology fits in an exact sequence

$$
0 \rightarrow \operatorname{Ext}_{\mathrm{MHS}}^{1}\left(\mathbb{Q}, H^{j-1}\left(C^{\prime}, \mathbb{V}\right)\right) \rightarrow H_{\mathcal{D}}^{j}\left(C^{\prime}, \mathbb{V}\right) \rightarrow \operatorname{Hom}_{\mathrm{MHS}}\left(\mathbb{Q}, H^{j}\left(C^{\prime}, \mathbb{V}\right)\right) \rightarrow 0
$$

Deligne-Beilinson cohomology of a higher dimensional variety $X=\bar{X}-D$ with coefficients in a PVHS $\mathbb{V}$ can be defined using Saito's mixed Hodge complex that generalizes Zucker's. 
The next result follows directly from the Manin-Drinfeld Theorem (Thm. 11.5) using the exact sequence (18.1).

Proposition 18.2. The DB-cohomology $H_{\mathcal{D}}^{j}\left(\mathcal{M}_{1,1}, S^{m} \mathbb{H}(r)\right)$ vanishes when $m$ is odd and when $j>2$. When $j=1$ it vanishes except when $m=2 n$ and $r=2 n+1$, in which case it is isomorphic to $\mathbb{Q}$. When $j=2$

$$
\begin{aligned}
H_{\mathcal{D}}^{2}\left(\mathcal{M}_{1,1}, S^{2 n} \mathbb{H}(r)\right)= & \operatorname{Ext}_{\mathrm{MHS}}^{1}\left(\mathbb{Q}, H^{1}\left(\mathcal{M}_{1,1}, S^{2 n} \mathbb{H}\right)(r)\right) \\
& \cong \operatorname{Ext}_{\mathrm{MHS}}^{1}(\mathbb{Q}, \mathbb{Q}(r-2 n-1)) \oplus \bigoplus_{f} \operatorname{Ext}_{\mathrm{MHS}}^{1}\left(\mathbb{Q}, M_{f}(r)\right)
\end{aligned}
$$

where $f$ ranges over the equivalence classes of $f \in \mathfrak{B}_{2 n+2}$.

As explained in Section 17.2.1, the real Frobenius $\mathcal{F}_{\infty}$ acts on $\mathcal{M}_{1,1}$ and on the local system $\mathbb{H}$. Since $\overline{\mathcal{F}}_{\infty}$ preserves the Hodge filtration, it acts on the complex used to compute $H_{\mathcal{D}}^{\bullet}\left(\mathcal{M}_{1,1}, S^{2 n} \mathbb{H}_{\mathbb{R}}(r)\right)$ and thus on the Deligne cohomology itself.

Corollary 18.3. For all $n>0$ and $r \in \mathbb{Z}$, there are natural $\overline{\mathcal{F}}_{\infty}$-equivariant isomorphisms

$$
H_{\mathcal{D}}^{2}\left(\mathcal{M}_{1,1}, S^{2 n} \mathbb{H}_{\mathbb{R}}(r)\right) \cong \operatorname{Ext}_{\mathrm{MHS}}^{1}(\mathbb{R}, \mathbb{R}(r-2 n-1)) \oplus \bigoplus_{f \in \mathfrak{B}_{2 n+2}} \operatorname{Ext}_{\mathrm{MHS}}^{1}\left(\mathbb{R}, V_{f}(r)\right) .
$$

These vanish when $r<2 n+2$. The first term on the right hand side corresponds to the Eisenstein series $G_{2 n+2}$.

18.2. Deligne-Beilinson cohomology of affine groups. Here we recall the definition and basic properties of the Deligne-Beilinson cohomology of an affine group from [21]. Suppose that $G$ is an affine $\mathbb{Q}$ group that is an extension of a reductive $\mathbb{Q}$ group $R$ by a prounipotent group $U$. Suppose that the coordinate $\operatorname{ring} \mathcal{O}(G)$ and its sub algebra $\mathcal{O}(R)$ are Hopf algebras in the category ind-MHS. Then the coordinate ring $\mathcal{O}(U)$ of $U$ is also a Hopf algebra in ind-MHS. This implies that its Lie algebra $\mathfrak{u}$ is a pronilpotent Lie algebra in pro-MHS. Suppose that $V$ is a Hodge representation (Defn. 8.2) of $G$. The Deligne-Beilinson cohomology of $G$ is defined by

$$
H_{\mathcal{D}}^{\bullet}(G, V):=\operatorname{Ext}_{\operatorname{HRep}(G)}(\mathbb{Q}, V) .
$$

This has a natural product: if $V_{1}, V_{2}$ are in $\operatorname{HRep}(G)$, there are natural multiplication maps

$$
H_{\mathcal{D}}^{\bullet}\left(G, V_{1}\right) \otimes H_{\mathcal{D}}^{\bullet}\left(G, V_{2}\right) \rightarrow H_{\mathcal{D}}^{\bullet}\left(G, V_{1} \otimes V_{2}\right) .
$$

The following result is proved in [21].

Proposition 18.4. For all $j \geq 0$, there is a short exact sequence

$$
0 \rightarrow \operatorname{Ext}_{\mathrm{MHS}}^{1}\left(\mathbb{Q}, H^{j-1}(G, V)\right) \rightarrow H_{\mathcal{D}}^{j}(G, V) \rightarrow \operatorname{Hom}_{\mathrm{MHS}}\left(\mathbb{Q}, H^{j}(G, V)\right) \rightarrow 0 .
$$

18.3. Extensions of VMHS over curves. Suppose that $\mathbb{H}$ is a PVHS over $C^{\prime}$. Fix a base point $x \in C^{\prime}$ and let $R_{x}$ be the Zariski closure of the monodromy action $\pi_{1}\left(C^{\prime}, x\right) \rightarrow$ Aut $H_{x}$. Denote the completion of $\pi_{1}(X, x)$ with respect to $\pi_{1}\left(C^{\prime}, x\right) \rightarrow R_{x}$ by $\mathcal{G}_{x}$. Theorem 8.3 implies that the category $\operatorname{MHS}\left(C^{\prime}, \mathbb{H}\right)$ is equivalent to $\operatorname{HRep}\left(\mathcal{G}_{x}\right)$, so that there is a natural isomorphism

$$
\operatorname{Ext}_{\mathrm{MHS}\left(C^{\prime}, \mathbb{H}\right)}^{\bullet}(\mathbb{Q}, \mathbb{V}) \cong H_{\mathcal{D}}^{\bullet}\left(\mathcal{G}_{x}, V_{x}\right) \text {. }
$$

Even more is true: 
Theorem $18.5([21])$. If $\mathbb{V}$ is an object of $\operatorname{MHS}\left(C^{\prime}, \mathbb{H}\right)$, then there are natural isomorphisms

$$
\operatorname{Ext}_{\mathrm{MHS}\left(C^{\prime}, \mathbb{H}\right)}(\mathbb{Q}, \mathbb{V}) \cong H_{\mathcal{D}}^{\bullet}\left(\mathcal{G}_{x}, V_{x}\right) \stackrel{\simeq}{\longrightarrow} H_{\mathcal{D}}^{\bullet}\left(C^{\prime}, \mathbb{V}\right)
$$

where $V_{x}$ denotes the fiber of $\mathbb{V}$ over the base point $x$. These isomorphisms are compatible with the natural products. ${ }^{26}$

Denote the category of admissible variations of MHS over $C^{\prime}$ by $\operatorname{MHS}\left(C^{\prime}\right)$. Since the Deligne cohomology $H_{\mathcal{D}}^{\bullet}\left(C^{\prime}, \mathbb{V}\right)$ does not depend on the choice of the basic variation $\mathbb{H}$ with $\mathbb{V} \in \operatorname{MHS}\left(C^{\prime}, \mathbb{H}\right)$, we have the following useful result.

Corollary 18.6 ([21]). For all admissible variations of $M H S$ over $C^{\prime}$, there is a natural isomorphism

that is compatible with products.

$$
\operatorname{Ext}_{\mathrm{MHS}\left(C^{\prime}\right)}^{\bullet}(\mathbb{Q}, \mathbb{V}) \cong H_{\mathcal{D}}^{\bullet}\left(C^{\prime}, \mathbb{V}\right)
$$

18.4. Extensions of variations of MHS over modular curves. Suppose that $\Gamma$ is a congruence subgroup of $\mathrm{SL}_{2}(\mathbb{Z})$. The following result follows from Corollary 18.6 and the Manin-Drinfeld Theorem (Thm. 11.5).

Proposition 18.7. If $m>0$ and $A$ is a Hodge structure, then

$$
\operatorname{Ext}_{\mathrm{MHS}\left(X_{\Gamma}\right)}^{1}\left(\mathbb{Q}, A \otimes S^{m} \mathbb{H}\right) \cong \operatorname{Hom}_{\mathrm{MHS}}\left(\mathbb{Q}, A \otimes H^{1}\left(X_{\Gamma}, S^{m} \mathbb{H}\right)\right) .
$$

When $m=0, \operatorname{Ext}_{\mathrm{MHS}\left(X_{\Gamma}\right)}^{1}(\mathbb{Q}, A)=\operatorname{Ext}_{\mathrm{MHS}}^{1}(\mathbb{Q}, A)$. If $A$ is a simple $\mathbb{Q}$-HS, then $\operatorname{Ext}_{\mathrm{MHS}\left(X_{\Gamma}\right)}^{1}\left(\mathbb{Q}, A \otimes S^{m} \mathbb{H}\right)$ is non-zero if and only if either $A=\mathbb{Q}(m+1)$ or $A \cong$ $M_{f}(m+1)$ for some Hecke eigen cusp form $f \in \mathfrak{B}_{m+2}(\Gamma)$.

This result can be interpreted as a computation of the group of normal functions (tensored with $\mathbb{Q}$ ) over $X_{\Gamma}$ associated to a PVHS of the form $\mathbb{V}=A \otimes S^{m} \mathbb{H}$. These are holomorphic sections of the bundle of intermediate jacobians associated to $\mathbb{V}$. The group of normal functions (essentially by definition) is isomorphic to $\operatorname{Ext}_{\mathrm{MHS}\left(X_{\Gamma}, \mathbb{H}\right)}^{1}(\mathbb{Z}, \mathbb{V})$. The normal functions constructed in Section 13.4 generate all simple extensions and normal functions in $\operatorname{MHS}\left(X_{\Gamma}, \mathbb{H}\right)$.

\section{Cup Products and Relations in $\mathfrak{u}^{\text {eis }}$}

In this section we show that Pollack's quadratic relations lift to relations in $\mathfrak{u}^{\text {eis }}$. In particular, we show that $\mathfrak{u}^{\text {eis }}$ is not free. Throughout, the base point is $\vec{v}=\partial / \partial q$, although most of the arguments are valid with any base point. As before, $H$ denotes the fiber of $\overline{\mathcal{H}}$ over $\vec{v}$. In this setup, $\mathbf{w}=2 \pi i \mathbf{b}$. We will omit the base point from the notation.

Recall from Section 17.3 that $\mathcal{D}$ denotes the image of the monodromy homomorphism $\mathcal{G} \rightarrow$ Autp. As before, we take $S^{2 n} H=S^{2 n}\left(\mathbf{b}^{2 n}\right)$. Let $\check{\mathbf{e}}_{2 n}$ be the element of

$$
H^{1}\left(\mathcal{G}, S^{2 n} H\right) \cong \operatorname{Hom}_{\mathrm{SL}(H)}\left(H_{1}\left(\mathfrak{u}, S^{2 n} H\right)\right)
$$

that takes the class of $\mathbf{e}_{2 n+2}$ to $\mathbf{b}^{2 n}$. Recall that the real Frobenius $\overline{\mathcal{F}}_{\infty}$ acts on $H_{\mathcal{D}}^{\bullet}\left(\mathcal{M}_{1,1}, S^{2 n} \mathbb{H}_{\mathbb{R}}(r)\right)$.

\footnotetext{
${ }^{26}$ There is a more general result which applies when $C^{\prime}$ is replaced by a smooth variety $X$ of arbitrary dimension. In that case, there is a natural homomorphism $H_{\mathcal{D}}^{\bullet}\left(\mathcal{G}_{x}, V_{x}\right) \rightarrow H_{\mathcal{D}}^{\bullet}(X, \mathbb{V})$ that is an isomorphism in degrees $\leq 1$ and injective in degree 2 . This is proved in [21].
} 
Lemma 19.1. For all $m>0$, the homomorphisms $\mathcal{G} \rightarrow \mathcal{G}^{\text {eis }} \rightarrow \mathcal{D}$ induce isomorphisms

$$
\begin{aligned}
H_{\mathcal{D}}^{1}\left(\mathcal{D}, S^{2 n} \mathbb{H}(2 n+1)\right) \stackrel{\simeq}{\longrightarrow} H_{\mathcal{D}}^{1}\left(\mathcal{G}^{\text {eis }}, S^{2 n} \mathbb{H}(2 n+1)\right) \\
\quad \stackrel{\simeq}{\longrightarrow} H_{\mathcal{D}}^{1}\left(\mathcal{G}, S^{2 n} \mathbb{H}(2 n+1)\right) \stackrel{\simeq}{\longrightarrow} H_{\mathcal{D}}^{1}\left(\mathcal{M}_{1,1}, S^{2 n} \mathbb{H}(2 n+1)\right)^{\overline{\mathcal{F}}_{\infty}} .
\end{aligned}
$$

Each of these groups is a 1-dimensional $\mathbb{Q}$ vector space. The first is spanned by $\check{\epsilon}_{2 n+2}$, the last by $\check{\mathbf{e}}_{2 n+2} / 2 \pi i$. The isomorphism identifies $\check{\epsilon}_{2 n+2}$ with

$$
\frac{2}{(2 n) !} \frac{\check{\mathbf{e}}_{2 n+2}}{2 \pi i} \text {. }
$$

Proof. For all $n>0$, each of the groups

$$
H^{1}\left(\mathcal{D}, S^{2 n} H\right), H^{1}\left(\mathcal{G}^{\text {eis }}, S^{2 n} H\right), H^{1}\left(\mathcal{G}^{\text {eis }}, S^{2 n} H\right)
$$

is isomorphic to $\mathbb{Q}(-2 n-1)$. The left-hand group is generated by $\check{\epsilon}_{2 n+2}$ and the right two groups by $\check{\mathbf{e}}_{2 n+2}$. Theorem 15.7 implies that the projections $\mathcal{G} \rightarrow \mathcal{G}^{\text {eis }} \rightarrow \mathcal{D}$ take $\check{\epsilon}_{2 n+2}$ to $2 \check{\mathbf{e}}_{2 n+2} / 2 \pi i(2 n)$ !, so that the homomorphisms induced by the projections are isomorphisms.

Proposition 18.4 implies that the projections $\mathcal{G} \rightarrow \mathcal{G}^{\text {eis }} \rightarrow \mathcal{D}$ induce isomorphisms

$$
H_{\mathcal{D}}^{1}\left(\mathcal{G}^{\text {eis }}, S^{2 n} \mathbb{H}(2 n+1)\right) \stackrel{\simeq}{\longrightarrow} H_{\mathcal{D}}^{1}\left(\mathcal{G}, S^{2 n} \mathbb{H}(2 n+1)\right) \cong \mathbb{Q}
$$

for all $n>0$. The corresponding class in Deligne cohomology is easily seen to be $\overline{\mathcal{F}}_{\infty}$ invariant.

Lemma 19.2. There is a natural inclusion

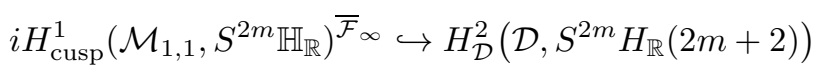

Proof. Since $\mathfrak{u}$ is free, $H^{2}\left(\mathcal{G}, S^{m} H\right) \cong H^{2}\left(\mathfrak{u}, S^{m} H\right)^{\mathrm{SL}(H)}$ vanishes for all $m>0$. The result follows from Proposition 18.4, the computation (Thm. 7.17) of $H^{1}(\mathfrak{u})$ and the isomorphism

$$
\begin{aligned}
\operatorname{Ext}_{\mathrm{MHS}}^{1}\left(\mathbb{R}, H_{\text {cusp }}^{1}\left(\mathcal{M}_{1,1}, S^{2 m} \mathbb{H}(2 m+2)\right)\right)^{\overline{\mathcal{F}}_{\infty}} & \\
& \cong i H_{\text {cusp }}^{1}\left(\mathcal{M}_{1,1}, S^{2 m} \mathbb{H}_{\mathbb{R}}\right)^{\overline{\mathcal{F}}_{\infty}}=\bigoplus_{f \in \mathfrak{B}_{2 m+2}} i V_{f}^{-} .
\end{aligned}
$$

which is well defined up to an even power of $2 \pi i$ that depends upon the choice of the first isomorphism.

By Corollary 18.3 , there is an $\overline{\mathcal{F}}_{\infty}$ invariant projection

$$
H_{\mathcal{D}}^{2}\left(\mathcal{M}_{1,1}, S^{2 n} \mathbb{H}_{\mathbb{R}}(2 n+2)\right) \rightarrow \operatorname{Ext}_{\mathrm{MHS}}^{1}\left(\mathbb{R}, V_{f}(2 n+2)\right) .
$$

The following computation is the key to proving that Pollack's quadratic relations are motivic.

Theorem 19.3 (Brown [3, Thm. 11.1], Terasoma [43, Thm. 7.3]). If $j, k>0$ and $n=j+k$ and if there is a cup form of weight $2 n+2$, then the image of the cup product

$$
\begin{aligned}
H_{\mathcal{D}}^{1}\left(\mathcal{M}_{1,1}, S^{2 j} \mathbb{H}_{\mathbb{R}}(2 j+1)\right) \otimes H_{\mathcal{D}}^{1}\left(\mathcal{M}_{1,1}, S^{2 k} \mathbb{H}_{\mathbb{R}}(2 k+1)\right) & \\
& \rightarrow H_{\mathcal{D}}^{2}\left(\mathcal{M}_{1,1}, S^{2 n} \mathbb{H}_{\mathbb{R}}(2 n+2)\right)
\end{aligned}
$$


is non-zero. More precisely, the composition of the cup product with the projection

$$
H_{\mathcal{D}}^{2}\left(\mathcal{M}_{1,1}, S^{2 n} \mathbb{H}_{\mathbb{R}}(2 n+2)\right) \rightarrow \operatorname{Ext}_{\mathrm{MHS}}^{1}\left(\mathbb{R}, V_{f}(2 n+2)\right)^{\overline{\mathcal{F}}_{\infty}} \cong V_{f, \mathbb{R}}^{-}
$$

is non-trivial for all $f \in \mathfrak{B}_{2 n+2}$.

As we shall show below, a direct consequence is that Pollack's quadratic relations hold in $\mathfrak{u}^{\text {eis }}$. Brown's period computations [3] are more detailed and imply that all of Pollack's relations lift from a quotient of $\operatorname{Der}^{0} \mathfrak{p}$ to relations in $\mathfrak{u}^{\text {eis }}$ and are therefore motivic. Full details will appear in [23].

Theorem 19.4. Pollack's quadratic relations (17.11) hold in $\mathrm{Gr}_{\bullet}^{W} \mathfrak{u}^{\mathrm{eis}}$. In particular, the pronilpotent radical $\mathfrak{u}^{\text {eis }}$ of $\mathfrak{g}^{\text {eis }}$ is not free.

We use the notation $\Gamma V:=\operatorname{Hom}_{\mathrm{MHS}}(\mathbb{Q}, V)$ to denote the set of Hodge classes of type $(0,0)$ of a MHS $V$.

Proof. Suppose that $n>0$. Since the Hodge structure $H_{\text {cusp }}^{1}\left(\mathcal{M}_{1,1}, S^{2 n} \mathbb{H}(2 n+2)\right)$ does not occur in $H^{1}\left(\mathfrak{u}^{\text {eis }}\right)$, Proposition 18.4 and the Manin-Drinfeld Theorem imply that the homomorphism

$$
\begin{aligned}
H_{\mathcal{D}}^{2}\left(\mathcal{G}^{\text {eis }}, S^{2 n} \mathbb{H}(2 n+2)\right) & \rightarrow H_{\mathcal{D}}^{2}\left(\mathcal{G}, S^{2 n} \mathbb{H}(2 n+2)\right) \\
& \cong \operatorname{Ext}_{\mathrm{MHS}}^{1}\left(\mathbb{Q}, H^{1}\left(\mathcal{M}_{1,1}, S^{2 n} \mathbb{H}(2 n+2)\right)\right) \\
& \rightarrow \operatorname{Ext}_{\mathrm{MHS}}^{1}\left(\mathbb{Q}, H_{\text {cusp }}^{1}\left(\mathcal{M}_{1,1}, S^{2 n} \mathbb{H}(2 n+2)\right)\right)
\end{aligned}
$$

induces a well-defined map

$$
r: \Gamma H^{2}\left(\mathcal{G}^{\mathrm{eis}}, S^{2 n} \mathbb{H}(2 n+2)\right) \rightarrow \operatorname{Ext}_{\mathrm{MHS}}^{1}\left(\mathbb{Q}, H_{\text {cusp }}^{1}\left(\mathcal{M}_{1,1}, S^{2 n} \mathbb{H}(2 n+2)\right)\right) .
$$

Compose this with the projection to

$$
\operatorname{Ext}_{\mathrm{MHS}}^{1}\left(\mathbb{R}, H_{\text {cusp }}^{1}\left(\mathcal{M}_{1,1}, S^{2 n} \mathbb{H}(2 n+2)\right)\right) \cong \bigoplus_{f \in \mathfrak{B}_{2 n+2}} V_{f, \mathbb{R}}
$$

to obtain a projection

$$
p: \Gamma H^{2}\left(\mathcal{G}^{\mathrm{eis}}, S^{2 n} \mathbb{H}(2 n+2)\right) \rightarrow \bigoplus_{f \in \mathfrak{B}_{2 n+2}} V_{f, \mathbb{R}}
$$

Consider the diagram

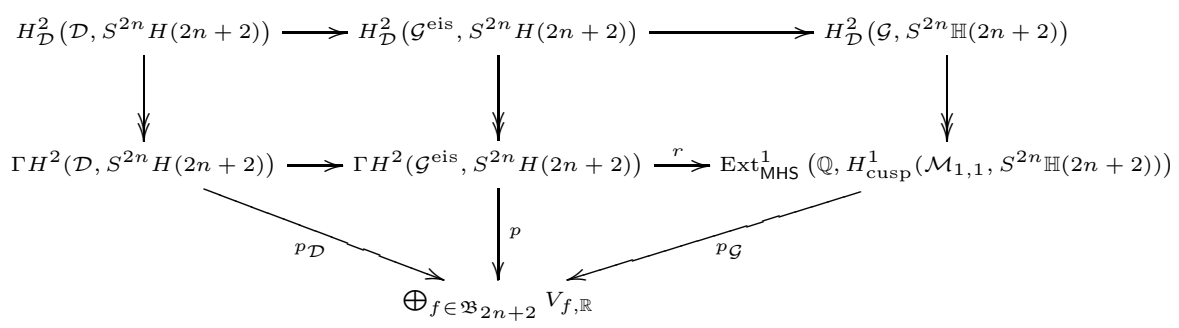

where $p_{\mathcal{D}}$ is the projection dual to the Pollack relations (Prop. 17.12), $p$ is the projection constructed above and $p_{\mathcal{G}}$ is the standard projection. Naturality implies that the top left square commutes; the right hand square commutes by the construction of $r$; the bottom right triangle commutes by the definition of $p$. The cup product computation (Thm. 19.3) implies that the sum of the two triangles commute. It follows that the diagram commutes. 
Suppose that $j, k>0$ satisfy $n=j+k$. Lemma 19.1 and Proposition 17.12 now imply that the composite of

$H^{1}\left(\mathcal{G}^{\mathrm{eis}}, S^{2 j} H(2 j+1)\right) \otimes H^{1}\left(\mathcal{G}^{\mathrm{eis}}, S^{2 k}, S^{2 k} H(2 j+1)\right) \rightarrow \Gamma H^{2}\left(\mathcal{G}^{\mathrm{eis}}, S^{2 n} H(2 n+2)\right)$

with the projection $p$ is given by the formula (17.14). The result now follows from the duality between cup product and quadratic relations.

Much of the discussion in this section can be generalized to modular curves $X_{\Gamma}$ where $\Gamma$ is a congruence subgroup of $\mathrm{SL}_{2}(\mathbb{Z})$. In particular, the prounipotent radical $\mathfrak{u}_{\Gamma}^{\text {eis }}$ is not free for all congruence subgroups.

Remark 19.5. This result implies that, when $\Gamma=\mathrm{SL}_{2}(\mathbb{Z})$, Manin's quotient $\mathfrak{u}_{B}$ of $\mathfrak{u}_{\Gamma}$ (cf. 13.2) is not a quotient of $\mathfrak{u}_{\Gamma}$ in the category of Lie algebras with a MHS. If it were, it would $\mathfrak{u}_{\Gamma}^{\text {eis }}$. But since $\mathfrak{u}_{B}$ is free, and since $\mathfrak{u}_{\Gamma}^{\text {eis }}$ is not, $\mathcal{U}_{B} \rightarrow \mathcal{U}_{\Gamma}^{\text {eis }}$ cannot be an isomorphism.

\section{REFERENCES}

[1] A. Beilinson, A. Levin: The elliptic polylogarithm, in Motives (Seattle, WA, 1991), 123-190, Proc. Sympos. Pure Math., 55, Part 2, Amer. Math. Soc., 1994.

[2] A. Borel, J.-P. Serre: Théorèmes de finitude en cohomologie galoisienne, Comment. Math. Helv. 39 (1964), 111-164.

[3] F. Brown: Multiple modular values for $\mathrm{SL}_{2}(\mathbb{Z})$, [arXiv:1407.5167]

[4] F. Brown, A. Levin: Multiple Elliptic Polylogarithms, [arXiv:1110.6917]

[5] J. Carlson, R. Hain: Extensions of variations of mixed Hodge structure, Theorie de Hodge, Luminy, Juin, 1987, Asterisque no. 179-180, 39-65.

[6] D. Calaque, B. Enriquez, P. Etingof: Universal KZB equations: the elliptic case, in Algebra, arithmetic, and geometry: in honor of Yu. I. Manin. Vol. I, 165-266, Progr. Math., 269, Birkhäuser, Boston, 2009, [arXiv:math/0702670]

[7] K.-T. Chen: Extension of $C^{\infty}$ function algebra by integrals and Malcev completion of $\pi_{1}$, Advances in Math. 23 (1977), 181-210.

[8] J. Conant: The Johnson cokernel and the Enomoto-Satoh invariant, preprint, June 2013. [arXiv: 1306.3698]

[9] J. Conant, M. Kassabov, K. Vogtmann: Hairy graphs and the unstable homology of $\operatorname{Mod}(g, s)$, $\operatorname{Out}\left(F_{n}\right)$ and $\operatorname{Aut}\left(F_{n}\right)$, J. Topol. 6 (2013), 119-153. [arXiv:1107.4839]

[10] J. Conant, M. Kassabov, K. Vogtmann: Higher hairy graph homology, preprint, August 2013. [arXiv:1308.3825].

[11] P. Deligne: Théorie de Hodge, II, Inst. Hautes Études Sci. Publ. Math. No. 40 (1971), 5-57.

[12] P. Deligne: Le groupe fondamental de la droite projective moins trois points, Galois groups over $\mathbb{Q}$ (Berkeley, CA, 1987), 79-297, Math. Sci. Res. Inst. Publ., 16, Springer, 1989.

[13] R. Hain: The geometry of the mixed Hodge structure on the fundamental group, Algebraic geometry, Bowdoin, 1985, 247-282, Proc. Sympos. Pure Math., 46, Part 2, Amer. Math. Soc., 1987.

[14] R. Hain: The de Rham homotopy theory of complex algebraic varieties, I, K-Theory 1 (1987), $271-324$.

[15] R. Hain: Hodge-de Rham theory of relative Malcev completion, Ann. Sci. École Norm. Sup., t. 31 (1998), 47-92.

[16] R. Hain: Infinitesimal presentations of the Torelli groups, J. Amer. Math. Soc. 10 (1997), 597-651.

[17] R. Hain: Iterated integrals and algebraic cycles: examples and prospects, Contemporary trends in algebraic geometry and algebraic topology (Tianjin, 2000), 55-118, Nankai Tracts Math., 5, World Sci. Publ., 2002.

[18] R. Hain: Relative weight filtrations on completions of mapping class groups, in Groups of Diffeomorphisms, Advanced Studies in Pure Mathematics, vol. 52 (May, 2008), 309-368, Mathematical Society of Japan. [arXiv:0802.0814] 
[19] R. Hain: Lectures on Moduli Spaces of Elliptic Curves, Transformation Groups and Moduli Spaces of Curves, pp. 95-166, Adv. Lect. Math. (ALM), 16, Int. Press, Somerville, MA, 2011. [arXiv:0812.1803]

[20] R. Hain: Notes on the Universal Elliptic KZB Equation, preprint, 2013. [arXiv:1309.0580]

[21] R. Hain: Deligne-Beilinson cohomology of affine groups, preprint, 2015.

[22] R. Hain, M. Matsumoto: Weighted completion of Galois groups and Galois actions on the fundamental group of $\mathbb{P}^{1}-\{0,1, \infty\}$, Compositio Math. 139 (2003), 119-167.

[23] R. Hain, M. Matsumoto: Universal mixed elliptic motives, preprint, 2015.

[24] R. Hain, G. Pearlstein: Tannakian fundamental groups of categories of variations of mixed Hodge structure, in preparation, 2015.

[25] R. Hain, S. Zucker: Unipotent variations of mixed Hodge structure, Invent. Math. 88 (1987), 83-124.

[26] R. Hain, S. Zucker: A guide to unipotent variations of mixed Hodge structure, Hodge theory (Sant Cugat, 1985), 92-106, Lecture Notes in Math., 1246, Springer, 1987.

[27] S. Lang: Introduction to Modular Forms, with appendixes by D. Zagier and Walter Feit.Corrected reprint of the 1976 original. Grundlehren der Mathematischen Wissenschaften, 222. Springer-Verlag, Berlin, 1995.

[28] A. Levin, G. Racinet: Towards multiple elliptic polylogarithms, unpublished preprint, 2007, [arXiv:math/0703237]

[29] Y. Manin: Iterated integrals of modular forms and noncommutative modular symbols, Algebraic geometry and number theory, 565-597, Progr. Math., 253, Birkhäuser, 2006.

[30] Y. Manin: Iterated Shimura integrals, Mosc. Math. J. 5 (2005), 869-881, 973.

[31] J. Morgan: The algebraic topology of smooth algebraic varieties, Inst. Hautes Études Sci. Publ. Math. No. 48 (1978), 137-204.

[32] G. Mostow: Fully reducible subgroups of algebraic groups Amer. J. Math. 78 (1956), 200-221.

[33] A. Pollack: Relations between derivations arising from modular forms, undergraduate thesis, Duke University, 2009. ${ }^{27}$

[34] M. Saito: Modules de Hodge polarisables, Publ. Res. Inst. Math. Sci. 24 (1988), 849-995.

[35] M. Saito: Mixed Hodge modules, Publ. Res. Inst. Math. Sci. 26 (1990), 221-333.

[36] W. Schmid: Variation of Hodge structure: the singularities of the period mapping, Invent. Math. 22 (1973), 211-319.

[37] J.-P. Serre: Lie algebras and Lie groups, 1964 lectures given at Harvard University, Second edition. LNM 1500. Springer-Verlag, 1992.

[38] J.-P. Serre: A course in arithmetic, Translated from the French. GTM 7, Springer-Verlag, 1973.

[39] C. Simpson: Higgs bundles and local systems, Inst. Hautes Études Sci. Publ. Math. No. 75 (1992), 5-95.

[40] J. Stallings: Homology and central series of groups, J. Algebra 2 (1965), 170-181.

[41] J. Steenbrink, S. Zucker: Variation of mixed Hodge structure, I, Invent. Math. 80 (1985), 489-542.

[42] D. Sullivan: On the intersection ring of compact three manifolds, Topology 14 (1975), 275277.

[43] T. Terasoma: Relative Deligne cohomologies and higher regulators for Kuga-Sato fiber spaces, manuscript dated January, 2011

[44] W. Wasow: Asymptotic expansions for ordinary differential equations. Pure and Applied Mathematics, Vol. XIV Interscience Publishers John Wiley \& Sons, 1965.

[45] S. Zucker: Hodge theory with degenerating coefficients, $L_{2}$ cohomology in the Poincaré metric, Ann. of Math. (2) 109 (1979), 415-476.

Department of Mathematics, Duke University, Durham, NC 27708-0320

E-mail address: hain@math.duke.edu

\footnotetext{
${ }^{27}$ Available at: http://dukespace.lib.duke.edu/dspace/handle/10161/1281
} 